\title{
Experimental and Numerical Investigation of Bio-Inpired Airfoil Trailing-Edge Designs for Noise Reduction
}

by

Yehia Salama, B.Sc.

A thesis submitted to the Faculty of Graduate and Postdoctoral Affairs in partial fulfillment of the requirements for the degree of

\section{Master of Applied Science}

in

Aerospace Engineering

\author{
Carleton University \\ Ottawa, Ontario
}

(C) 2021

Yehia Salama 


\section{Abstract}

Embedded Large Eddy Simulations (ELES) are employed in tandem with the Ffowcs Williams-Hawkings (FW-H) aeroacoustic model to investigate the aerodynamics and tonal noise of NACA0012 airfoils having different bio-inspired noise-suppressing trailing-edge (TE) configurations. Various designs, such as standard sawtooth serrations, surface finlets, finned serrations and slanted-root sawtooth serrations are studied and compared. The different designs are shown to leverage different noise-suppressing flow mechanisms. The effects of changing standard serration amplitude and wavelength on the radiated tonal peak is studied. Experimental results suggest that noise reduction for surface finlets is dependent on the airfoil angle of attack. Slanted-root serrations are shown to alter the flow-field and suppress unwanted tonal peaks. ELES results are compared with experimental measurements, with good overall agreement. ELES is demonstrated to be a reasonable alternative to the currently-used more computationally demanding, full LES or direct numerical simulation approaches. 
To my mother, may her soul rest in peace. 


\section{Acknowledgements}

This project would not have been possible without the support of many people. I would like to thank my supervisor, Prof. Joana Rocha, for the support and guidance she provided. I am grateful for the numerous discussions we had, and the freedom I was given to explore my research interests. Thank you for your patience, enthusiasm and immense knowledge. I am certain that working alongside her has improved the quality of my work, and taught me valuable skills that will allow me to excel in my future as a researcher.

I would like to thank Neil and Bruce for making it possible to work remotely during the past two years, allowing me to pursue my research despite the technical challenges imposed by the pandemic. I would also like to thank Alex and Kevin for their assistance on the design and fabrication of the experimental parts involved in wind tunnel testing.

Thank you to my fellow research colleagues and friends, Nick and Basim. I am grateful for your suggestions and the stimulating discussion we had about my research.

Finally, I want to acknowledge the tremendous support that I have received from my family and friends. Thank you for inspiring me and making me believe that anything is achievable through hard work and dedication. I wouldn't be in the position I am today without their continuous love and encouragement. Thanks to everyone who helped and believed in me, I am grateful to have you in my life. 


\section{Table of Contents}

Abstract............................................................................................................................ iii

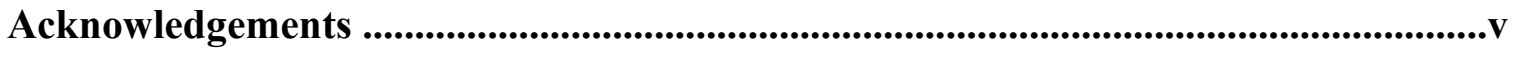

Table of Contents .............................................................................................................. vi

List of Tables .................................................................................................................................. viii

List of Figures............................................................................................................................ ix

Nomenclature ................................................................................................................................... xi

Introduction ..................................................................................................................................1

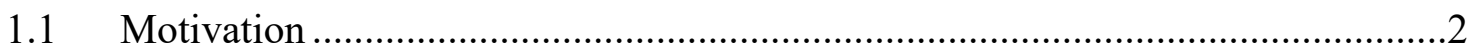

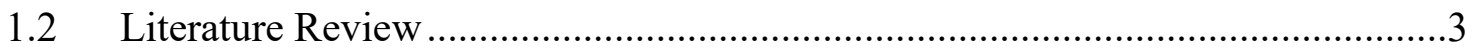

1.2.1 A Review of Airfoil Noise Generation Mechanisms .......................................3

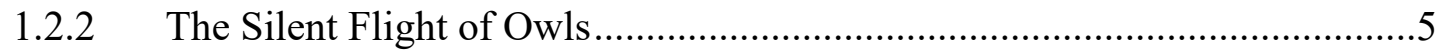

1.2.3 Leading and Trailing Edge Serrations ………...............................................

1.2.4 Surface Finlets …………………………………................................11

1.2.5 Aeroacoustic Analogies ........................................................................13

1.2.6 Aerodynamic Noise Prediction ................................................................14

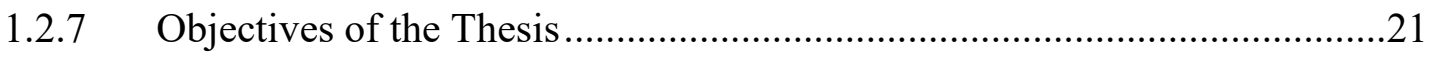

1.2.8 Research Contributions .......................................................................22

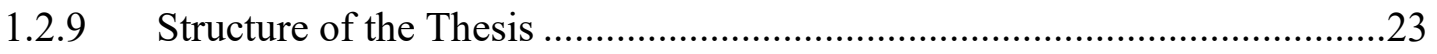

Numerical Methods ...............................................................................................24

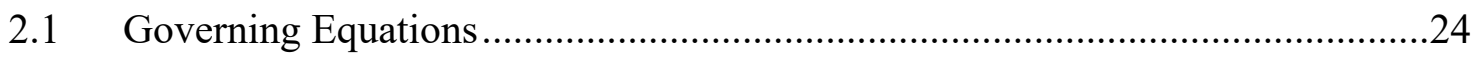




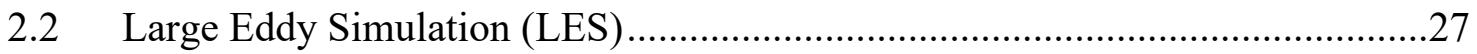

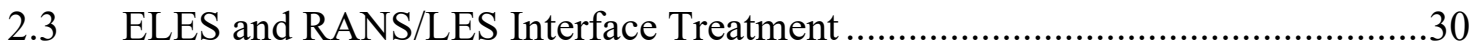

2.4 The Ffowcs Williams and Hawkings (FW-H) Aeroacoustic Analogy...............32

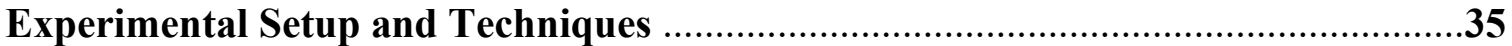

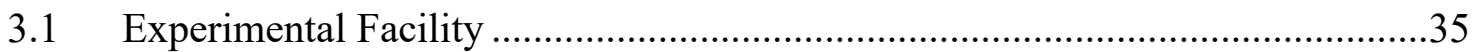

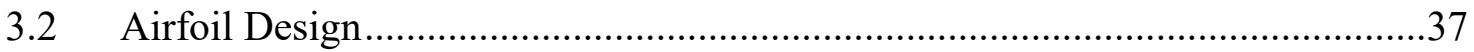

3.3 Wind Tunnel Setup and Experimental Techniques.......................................40

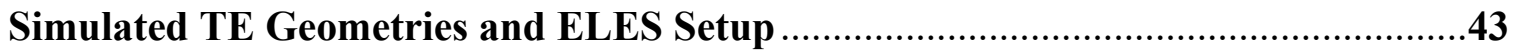

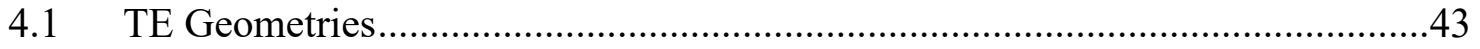

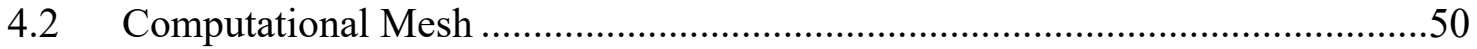

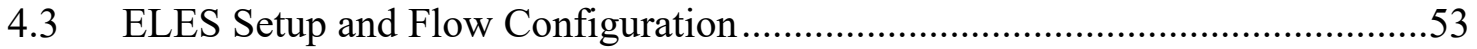

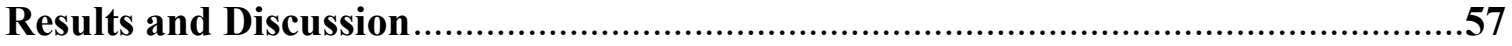

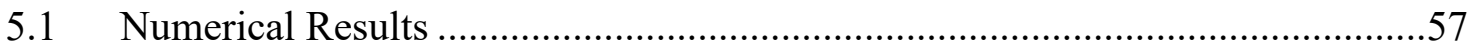

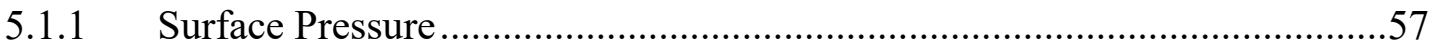

5.1.2 Turbulence Kinetic Energy Distribution.................................................61

5.1.3 Wake Vorticity and Characteristics ....................................................67

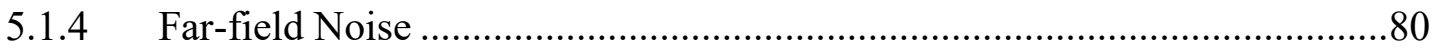

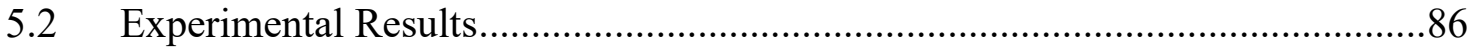

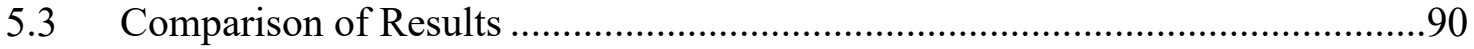

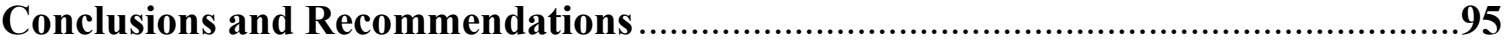

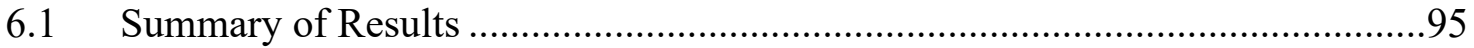

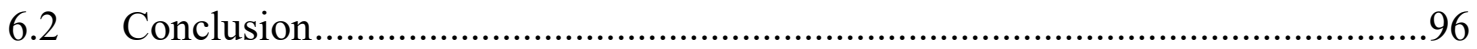

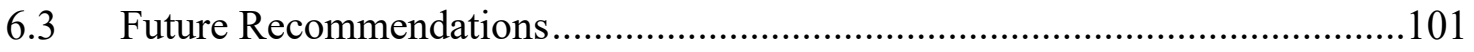

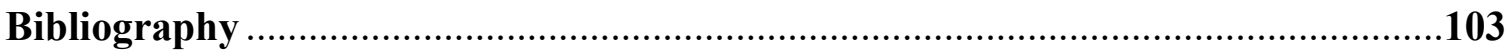

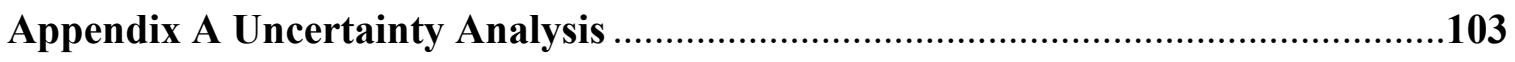




\section{List of Tables}

Table 1: Airfoil noise-generating mechanisms.................................................. 4

Table 2: Characteristics of flat TE airfoils. ....................................................... 44

Table 3: Geometric characteristics of standard TE serrations........................... 45

Table 4: Summary of all presented simulations. ............................................... 48

Table 5: Characteristics of meshes used in mesh convergence study. ................ 51

Table 6: Number of elements in each computational mesh. ............................ 53

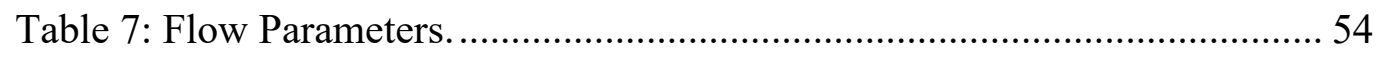

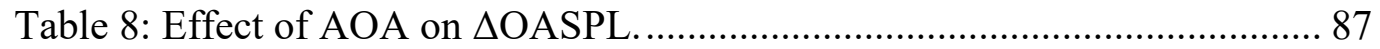

Table 9: Summary of standard serration characteristics and corresponding tonal

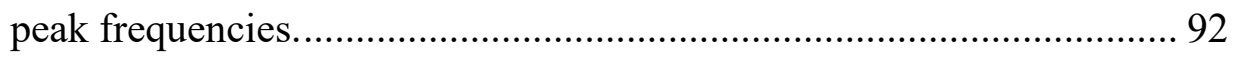




\section{List of Figures}

Figure 1: Flow conditions producing airfoil self-noise, taken from [5].............. 4

Figure 2: Details of the three special feather adaptions of the barn owl taken from

[13]: (A) leading-edge serrations; (B) trailing-edge fringes; and (C)

soft downy coating surface. ........................................................... 6

Figure 3: Carleton University medium speed wind tunnel configuration. ........... 36

Figure 4: The aeroacoustic test section and anechoic chambers, as seen from

above

Figure 5: Drawing sheet showing details of the manufactured finned TE, units in $\mathrm{mm}$, not to scale.

Figure 6: Manufactured finned NACA0012 airfoil TE.................................... 39

Figure 7: Fully assembled NACA0012 airfoil with TE finlets. ......................... 40

Figure 8: NACA0012 airfoil with the finned TE attached, as seen with the upper aluminum panel removed. Flow direction is from left to right.......... 41

Figure 9: AOA configuration and microphone location................................. 42

Figure 10: General model of standard TE serrations, not to scale. .................... 45

Figure 11 : General model of TE finlets, not to scale. …................................... 46

Figure 12: General model of finned TE serrations, not to scale........................ 47

Figure 13: General model of slanted-root TE serrations, not to scale................ 48

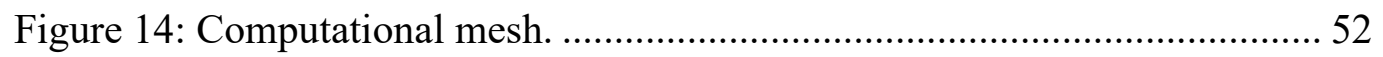

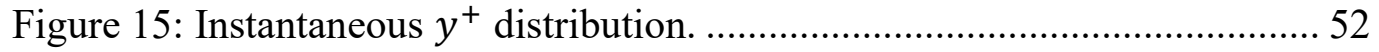

Figure 16: Schematic illustration of the segregated modeling domains.............. 54

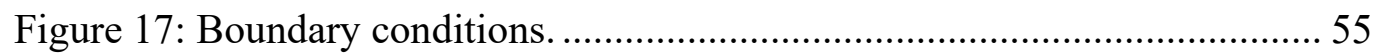

Figure 18: $C p$ distribution on airfoil surface ............................................. 58 


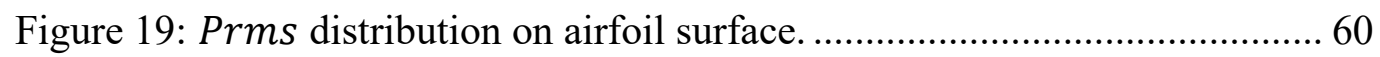

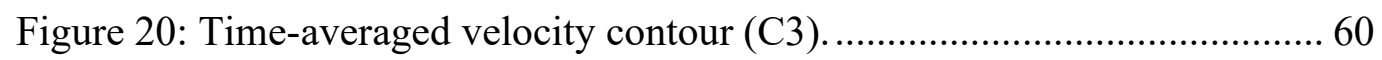

Figure 21: Instantaneous TKE distribution for a flat TE airfoil........................... 62

Figure 22: Instantaneous TKE distribution for standard TE serrations................ 63

Figure 23: Instantaneous TKE distribution for TE finlets.................................... 64

Figure 24: Instantaneous TKE distribution for finned TE serrations................... 65

Figure 25: Difference in flow channels for cases C3 (top) and C4 (bottom)...... 65

Figure 26: Instantaneous TKE distribution for slanted-root TE serrations......... 67

Figure 27: Lift-coefficient history of C2.3 using two different meshes............... 69

Figure 28: Lift-coefficient history for $\mathrm{C} 1.2, \mathrm{C} 2.1, \mathrm{C} 2.2$ and $\mathrm{C} 2.3 \ldots \ldots \ldots \ldots \ldots \ldots \ldots . . . . .70$

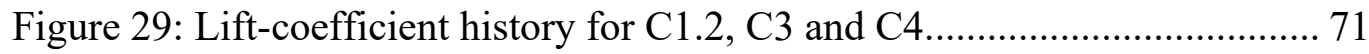

Figure 30: Lift-coefficient history for C2.1, C2.2 and C5 ................................ 72

Figure 31: Instantaneous wake vorticity magnitude........................................... 74

Figure 32: Instantaneous Q-Criterion coloured by $\omega z$........................................ 77

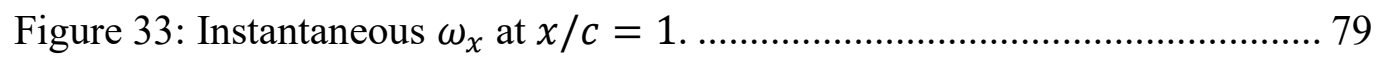

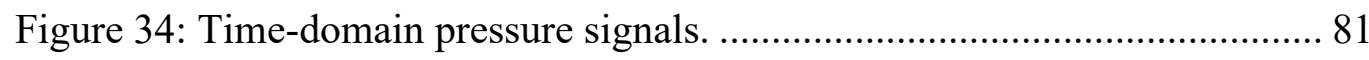

Figure 35: Frequency-domain noise signals of $\mathrm{C} 1.2, \mathrm{C} 2.1, \mathrm{C} 2.2$ and $\mathrm{C} 2.3 \ldots \ldots . .81$

Figure 36: Frequency-domain noise signals of $\mathrm{C} 2.3$ using different meshes...... 81

Figure 37: Frequency-domain noise signals of $\mathrm{C} 1.2$ and $\mathrm{C} 3$............................. 81

Figure 38: Frequency-domain pressure signals for $\mathrm{C} 2.3, \mathrm{C} 3$ and $\mathrm{C} 4$. ................ 81

Figure 39: Frequency-domain pressure signals for $\mathrm{C} 2.1, \mathrm{C} 2.3$ and $\mathrm{C} 5$............... 81

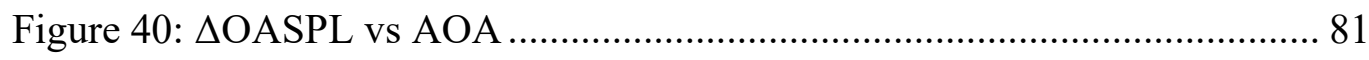

Figure 41: Experimentally obtained SPL, AOA $=0^{\circ}, 5^{\circ}$ and $-5^{\circ} \ldots \ldots \ldots \ldots \ldots \ldots \ldots . . . . . . . . . .11$

Figure 42: Experimentally obtained SPL, $\mathrm{AOA}=10^{\circ}$ and $-10^{\circ} \ldots \ldots \ldots \ldots \ldots \ldots \ldots \ldots . . . . . . . . . . . .81$

Figure 43: Experimentally obtained SPL, straight TE ....................................... 81

Figure 44: Experimentally obtained SPL, finlets …………………………….... 81

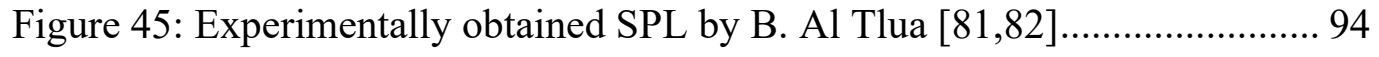




\section{Nomenclature}

$$
\begin{aligned}
& A O A=\text { angle of attack } \\
& C F D=\text { computational fluid dynamics } \\
& c \quad=\quad \text { airfoil chord length, } m \\
& C_{p}=\text { pressure coefficient } \\
& B L=\text { boundary layer } \\
& T B L=\text { turbulent boundary layer } \\
& d t \quad=\quad \text { time step, } s \\
& t \quad=\quad \text { time, } s \\
& f_{s} \quad=\quad \text { sampling frequency, } \mathrm{Hz} \\
& \varepsilon \quad=\quad \text { serration root bluntness, } m \\
& 2 h=\text { serration amplitude, } m \\
& \lambda=\text { serration wave length, } m \\
& f \quad=\quad \text { frequency, } \mathrm{Hz} \\
& u, v, w=\quad \text { fluid velocity, } \mathrm{m} / \mathrm{s} \\
& P \quad=\quad \text { fluid pressure, } P a \\
& M \quad=\quad \text { Mach number } \\
& N S=\text { Navier Stokes equations } \\
& R e \quad=\quad \text { Reynolds number } \\
& R e_{c}=\text { chord-based Reynolds number } \\
& R M S=\text { root mean square } \\
& \mu \quad=\quad \text { dynamic viscosity, } \mathrm{Ns} / \mathrm{m}^{2} \\
& v=\text { kinematic viscosity, } \mathrm{m}^{2} / \mathrm{s} \\
& L \quad=\quad \text { length, } m \\
& \text { LES = large eddy simulation }
\end{aligned}
$$




$\begin{array}{lll}E L E S & = & \text { embedded large eddy simulation } \\ Q & = & \text { second invariant of velocity gradient tensor } \\ R A N S & = & \text { Reynold's averaged Navier Stokes equations } \\ \rho & = & \text { density, } \mathrm{kg} / \mathrm{m}^{3} \\ S & = & \text { airfoil span, } \mathrm{m} \\ \mathrm{SGS} & = & \text { Sub-grid-scale } \\ \mathrm{TE} & = & \text { trailing-edge } \\ \mathrm{LE} & = & \text { leading-edge } \\ T_{F T} & = & \text { through-flow time, } \mathrm{s} \\ V M & = & \text { Vortex method } \\ \omega & = & \text { vorticity, } 1 / \mathrm{s}\end{array}$

Subscripts

$\infty=$ free-stream quantity

$r m s=$ root mean square 


\section{Chapter 1}

\section{Introduction}

With the world's noise pollution levels increasing, aerodynamic noise reduction has been steadily gaining the attention of the research community. Over the past decades, noise pollution from aviation has increased, disturbing the integrity of natural ecosystems and putting them at risk [1]. Elevated noise levels can lead to stress and sleep disruption, which can harm the mental and physical well-being of humans [2]. Governments and organizations are progressively applying stricter regulations that aim to reduce the environmental noise footprint. The United Nations International Civil Aviation Organization (ICAO) identified aircraft noise as the most significant cause of adverse community reactions related to the operation and expansion of airports [3]. The Committee of Aircraft Noise (CAN) developed the first standard on aircraft noise (CAN 1971) in order to reduce overall noise pollution and promote the best noise reduction for new aircraft entering services. [4]. When considering the overall noise generated by the numerous aircraft components, the airfoil trailing-edge (TE) was found to be a major contributor [5].

In parallel, global warming has led a universal push towards sustainability. Wind turbines have been growing in popularity as a sustainable form of energy generation. However, a major downside of wind turbines which prevents their widespread use is the noise pollution created by the rotating blades. The largest contributor to this noise pollution is that generated by the TE of wind turbine blades [6]. In general, TE noise is produced when turbulent air within the turbulent boundary layer (TBL) is convected past the airfoil TE and into the wake.

For TE noise reduction, barn owls have been used as a biomimicry candidate. Biomimicry is the practice of using nature as inspiration when attempting to create new designs. Since barn owls are known to be nature's most 
silent bird during flight [7], the morphological features of their wings are applied on NACA0012 airfoils in order to reduce TE noise. The advancement and design of new, quieter airfoils requires proper modeling and understanding of the aerodynamic flow mechanisms responsible for sound generation, as it relates the pressure fluctuations of the near-field to the radiated noise of the far-field. The Ffowcs Williams-Hawkings (FW-H) model [8] was developed to decouple sound generation from its propagation, replacing complex acoustic processes by equivalent acoustic sources, thus eliminating the need to fully resolve the far-field. This revolutionary approach allowed for the development of hybrid computational methods that can serve as practical alternatives to the computationally expensive full resolution of the flow field. Embedded Large Eddy Simulations (ELES) are chosen and employed in tandem with the FW-H model to understand the flow around bio-inspired airfoils and relate it to the radiated far-field noise. Experimental work is done to verify the applicability of the proposed method and provide accurate acoustic measurements.

\subsection{Motivation}

In 1934, R. R. Graham recognized the potential of using birds as a reference to improve the design of modern airplanes and identified owls as a biomimicry candidate to achieve silent flight [7]. Soon after, numerous researchers and engineers followed in his footsteps and worked on developing practical, bioinspired airfoil designs. Noise prediction models are needed in the design phase of those airfoils, to help ensure that the finished manufactured products have acceptable characteristics. The development of such prediction models is not a trivial task. In the past, mainly statistical and semi-empirical models were used for this purpose. The development of accurate computational models for the simulation of air flow around bio-inspired airfoils remains a challenging task, despite the recent technological advancements. This is mainly due to the large computational 
power required. To be useful as a design tool, such models must be sufficiently simple, yet comprehensive enough to capture all the physical mechanisms of interest, as well as provide reliable short-response-time noise prediction methods for industrial application and optimization.

In this context, the objective of this thesis is to provide an alternative to the currently-used computationally prohibitive simulation models, and use it to advance the fundamental understanding of bio-inspired airfoil TE designs. ELES was chosen and its ability to predict far-field noise and near-field hydrodynamics is assessed. Results are compared against published literature, and mesh convergence tests are performed to establish faith in the predictions. The model is then used to investigate the flow and noise characteristics of seven different TE configurations on a NACA0012 airfoil.

\subsection{Literature Review}

This section presents the state-of-the art review related to the development of highfidelity computational fluid dynamics (CFD) simulations, as well as the design of new, quieter airfoils. This review also discusses the primary concepts needed for the development of a suitable computational model to predict the far-field radiated noise for the designed airfoils.

\subsubsection{A Review of Airfoil Noise Generation Mechanisms}

In order to develop fundamental understanding of the numerous airfoil selfnoise mechanisms, research has been conducted and supported by the NASA Langley Research Center. In 1989, Thomas F. Brooks, D. Stuart Pope and Michael A. Marcolini [5] published an interpretation of the physical mechanisms 
responsible for noise generation. Airfoil self-noise is primarily caused by the interaction between the airfoil itself and the turbulence produced in its boundary layer and near wake. Five airfoil self-noise generating mechanisms were identified and listed in Table 1. Figure 1 further illustrates the flow conditions responsible for self-noise.

Table 1: Airfoil noise-generating mechanisms

\begin{tabular}{cl}
\hline $\begin{array}{c}\text { At high Re } \\
\text { (Figure 1a) }\end{array}$ & $\begin{array}{l}\text { Turbulent boundary layers (BLs) develop over most of } \\
\text { the airfoil and noise is generated as this turbulence goes } \\
\text { over the trailing edge. }\end{array}$ \\
\hline $\begin{array}{c}\text { At low Re } \\
\text { (Figure 1b) }\end{array}$ & $\begin{array}{l}\text { Predominantly laminar BLs develop over the airfoil } \\
\text { surface and their instabilities result in vortex shedding } \\
\text { and noise radiation, as flows go past the trailing edge. }\end{array}$ \\
\hline $\begin{array}{c}\text { If the trailing } \\
\text { edge is blunt } \\
\text { (Figure 1c) }\end{array}$ & $\begin{array}{l}\text { Vortex shedding in the small separated flow region is } \\
\text { another source of noise. }\end{array}$ \\
\hline $\begin{array}{c}\text { Near the wing tips } \\
\text { (Figure 1d) }\end{array}$ & $\begin{array}{l}\text { The formation of vortices near the wing tips produces } \\
\text { highly turbulent flows can emit noise. }\end{array}$ \\
\hline $\begin{array}{c}\text { At nonzero angles } \\
\text { of attack }\end{array}$ & $\begin{array}{l}\text { Separation may occur near the trailing edge, producing } \\
\text { noise due to the shed turbulent vorticity. At high angles } \\
\text { of attack and in stall, large-scale flow separation causes } \\
\text { the flow to emit low frequency noise similar to that of a } \\
\text { bluff body in flow. }\end{array}$ \\
\hline
\end{tabular}

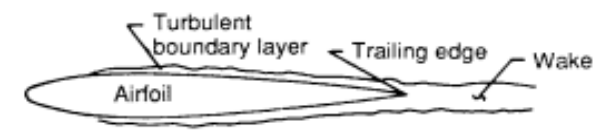

a) At high $\mathrm{Re}$

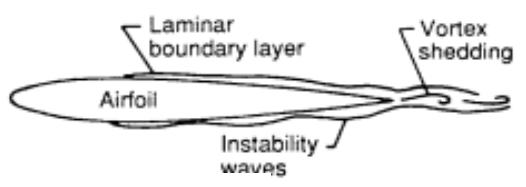

b) At low $\mathrm{Re}$

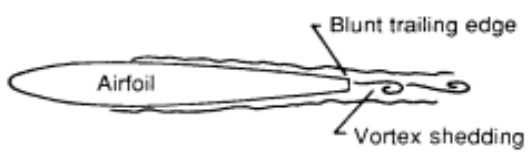

c) If the TE is blunt

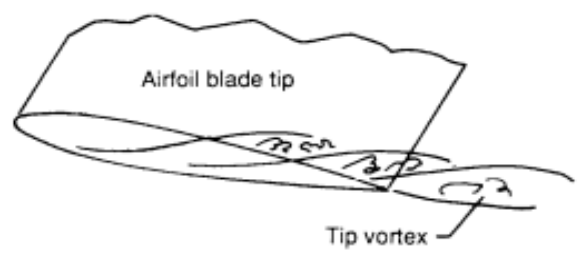

d) Near the wing tips

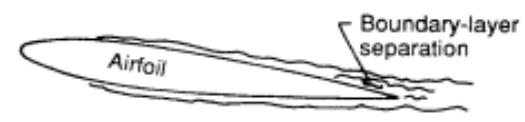

e) At nonzero AOAs

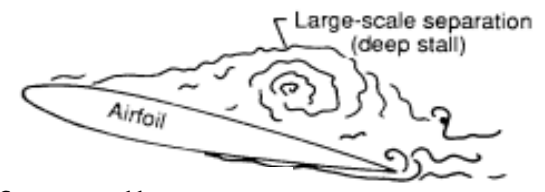

f) In stall

Figure 1: Flow conditions producing airfoil self-noise, taken from [5]. 
By combining their understanding of the underlying physics with biomimicry, researchers and engineers were able to develop many creative designs with better noise characteristics. The following subsection discusses the use of biomimicry for TE noise reduction purposes, and why barn owls were specifically chosen as biomimicry candidates.

\subsubsection{The Silent Flight of Owls}

Biomimicry investigates the structure and working principles of biological creatures in nature, and tries to translate these concepts to render engineering technologies more efficient, and/or to invent superior instruments aiming to solve complex human problems. It is difficult to predict how the flow of air will act in a certain setup, or how it should act, in order to decrease the radiated noise. The reason behind the complexity is the high nonlinearity of the governing equations. Therefore, engineers were drawn to nature looking for innovative ideas. In 1934, R. R. Graham [7] recognized the potential of using living birds as a reference to render modern airplanes more efficient. By observing the differences and flight tactics of Barn Owls (Tyto alba) and other birds, Graham concluded that noisesupressing designs are indeed possible, emphasizing the importance of using birds as a guide to future developments.

The wings of barn owls differ from those of all other groups of birds, due to three main noise reducing peculiarities [7]:

i. The leading-edge (LE) comb: A remarkably stiff comb-like fringe exists on the front margin of every feather.

ii. The trailing-edge (TE) fringe: A fringe resembling that of a shawl spans along the trailing edge of the main wing. 
iii. The downy upper surface: Certain parts of the upper surface are covered with a short, fine down.
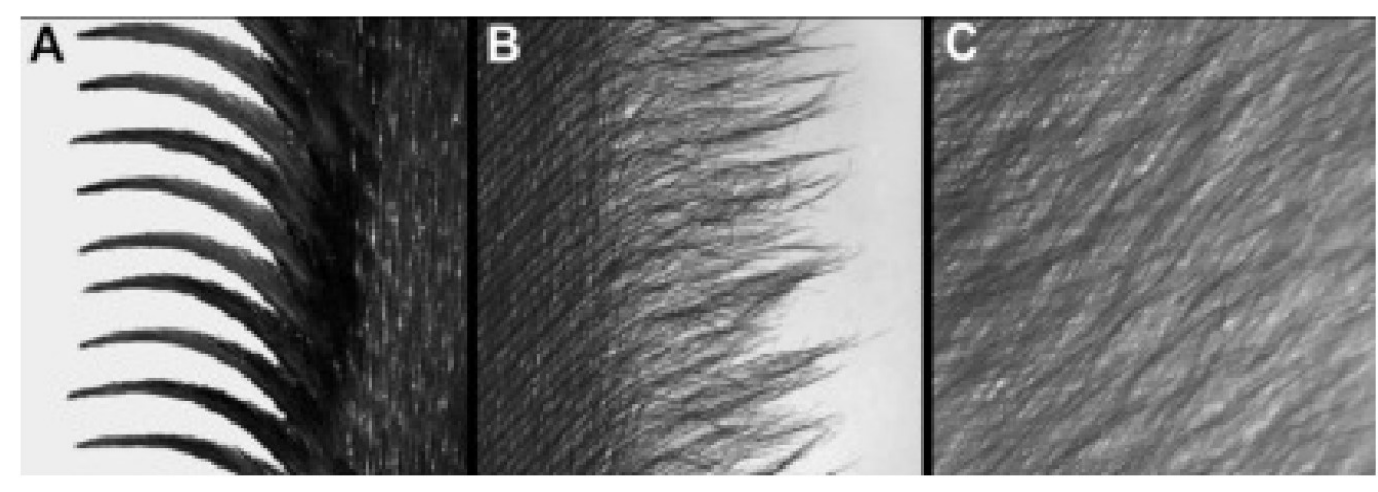

Figure 2: Details of the three special feather adaptions of the barn owl taken from [13]: (A) leading-edge serrations; (B) trailing-edge fringes; and (C) soft downy coating surface.

Hertel [8] theorized that the "compliant" downy upper surface could be acting as a cushion allowing the feathers to slide silently thus preventing noise. Gruschka Borchers and Coble [10] observed that the sound pressure level produced by the "Strix varia" owl is not audible to human beings at a distance of 3 metres or more. With the help of Kroeger and Helvey, Gruschka [11] led an extensive aerodynamic, aeroacoustic and bionics investigation looking to discover novel mechanisms to reduce the noise associated with aircraft flight. The proposed findings were later confirmed by the interpretation provided by Brooks et al. in 1989 [5]. In 1998, Lilley [12] confirmed the three main noise suppression mechanisms in owl wings previously addressed by Graham [7]. Lilley then discussed the aerodynamic characteristics of each of these devices and proposed explanations as to how the flow dynamics vary, which leads to noise reduction in the range of frequencies above $2000 \mathrm{~Hz}$. According to him:

i. The comb-shaped LE feathers behave as closely spaced co-rotating vortex generators, creating streamwise vortices which lead to a reduction in BL thickness and in the volume of turbulence crossing the trailing edge.

ii. The TE fringe resembles a serrated edge, which suggests the reduction or even elimination of TE scattering. 
iii. Thanks to the small diameter of its fibers, the compliant velvety surface of the wing acts as a bypass mechanism for energy dissipation at frequencies smaller than the conventional dissipation range of frequencies associated with viscous damping. In other words, the fibers absorb energy from the small-scale noiseemitting eddies, thus silencing them.

In 2007, Bachmann et al. [13] initiated a systematic analysis of wing morphology and verified the aforementioned specializations in owl wings, recommending their use as a source for noise-reducing applications on any surfaces subjected to flow fields. Having recognized the potential of mimicking barn owl feather structures in the aviation industry, Wagner and Bachmann [14] presented a detailed quantitative three-dimensional characterization of natural wing serrations. In 2017, Wagner et al. [15] published a comprehensive review of the morphological adaptations of owl that are responsible for their silent flight and discussed their aerodynamic properties, from early observations to recent advancements. It was concluded that the LE serrations and the velvet-like surface of the wing influence noise reduction and improve aerodynamic performance. Furthermore, the fringed trailing edge of the wing has been shown to reduce trailing edge noise. The following two subsections aim to isolate and analyse these morphological features and explore how they can be practically applied on solid airfoils.

\subsubsection{Leading and Trailing Edge Serrations}

After the noise-suppressing features of owl wings were better understood, the following step was and has been to implement them onto airfoils. This allows their efficiency as noise suppressors and their effects on aerodynamic flow properties to be studied.

Arndt \& Nagel experimentally showed that 'reduction in noise is possible with specific LE configurations and running conditions' [16], by introducing LE serrations to rotors. Results showed that LE serrations increase turbulence on the airfoil's upper surface, which in turn aids to reduce the peak root mean square 
(RMS) pressures that would otherwise be produced at low Reynold's number (Re) by 2D laminar bubbles very close to a flat LE. Alan Hersh et al. [17] presented a series of extensive studies on the application of LE serrations as a device for noise reduction in low $R e$ flows, and showed tonal noise suppression at low angles of attack with the serrations properly located, as well as broadband noise attenuation at high angles of attack corresponding to stall. Klän, Klaas and Schröder [18] showed that the magnitude of the impact of LE serrations on the flow field depends on different parameters, like the Re, angle of attack (AOA), the length of the serrations and the angle between them. The authors confirmed the effect of the LE serrations on flow transition at low $R e$, supporting the findings of Schwind \& Allen [19]. Narayanan et al. [20] investigated the use of sinusoidal LE serrations to reduce the broadband noise generated from the interaction between an airfoil's LE and the impinging turbulence. Results showed insignificant noise reduction at low frequencies but a significant one in the mid frequency range $(500 \mathrm{~Hz}-8 \mathrm{kHz})$ for all the cases studied. The noise reduction results were also compared to a NACA65 airfoil with the same LE serrations and they were found to be lower for the airfoil with a maximum reduction of around $7 \mathrm{~dB}$ compared with about $9 \mathrm{~dB}$ for the flat plates. It was concluded that the sound power reduction level is sensitive to the amplitude of the LE serrations but less sensitive to the serration wavelength. Thus, this paper identified the LE serration amplitude as a key parameter for improving noise reduction levels.

Herr and Dobrzynski [21] experimentally investigated the aerodynamic and aeroacoustic characteristics of TE brush extensions in an open jet wind tunnel. For solid TE configurations, the authors recognized two different noise spectra compositions; broadband noise from TBL flow across the TE and spectral narrowband peaks related to periodic vortex shedding at the TE. The flow permeable edge brush extensions used were found to be an effective means of reducing both the narrowband bluntness noise as well as the broadband TBL-TE noise generated through mitigating the "discontinuity", which is the abrupt change in boundary conditions of a solid TE interacting with the TBL flow. The authors assumed that this reduction is attributed to viscous damping of turbulent flow 
pressure amplitudes in the flow-permeable brush area, and that a transformation of spanwise vorticity into streamwise vorticity might be another contributor. This is an indication that the noise reduction capabilities of the brush edges are induced by local effects on the flow field in the source area, rather than a reduced sound radiation efficiency. Longer brushes were more effective and the model chord length had no significant impact on the brush devices' effectiveness. The authors also attributed the observed spectral peaks that are the result of vortex shedding in cases having blunt TE configurations.

In 2014, Gharali et al. [22] used PIV to study the effects of TE serrations on an oscillating SD7037 airfoil with a Re of 40,000 during deep stall. The goal was to investigate the effect of TE serrations on vortices. The results showed that TE serrations had no effect on the LE vortices, meaning that primary effects lie within another structure, such as TE vortices. It was also observed that the vortex shedding frequency is decreased when the airfoil is modified by serrations, and that the vortices themselves are diffused faster. Through wind tunnel testing, Liang et al. [23] examined the effect of different fan-vane trailing edge serrations on the noise produced. It was confirmed that TE serrations are a suitable noise reduction device even for fan-vane noise. Gruber et al. [24] experimentally showed that more complex TE serrations could improve broadband TE noise reduction. Three different trailing edges were analysed, slits, sawtooth serrations with holes and slitted sawtooth serrations. All treatments were manufactured from stiff cardboard and attached to the trailing edge of a NACA65(12)-10 airfoil. The sawtooth serration with holes provided relatively poor noise reductions of up to maximum 2 $\mathrm{dB}$, while an effective broadband noise reduction of $5 \mathrm{~dB}$ was reported for slitted serrations, which also suppressed the expected high frequency noise increase that was observed in the case of sawtooth serrations. The results show that minimizing both the width of the slits, and the distance between adjacent slits per sawtooth also improved noise attenuation. Chong et al. [25] experimentally investigated the feasibility of employing different serrated TE configurations to reduce the noise produced by a NACA0012 airfoil. For the case of an untripped boundary layer, testing velocities of up to $60 \mathrm{~m} / \mathrm{s}$ and an angle of attack of $4.2^{\circ}$, it was shown that 
sound power reduction of up to $30 \mathrm{~dB}$ is possible for the instability tonal noise. When the airfoil surface is tripped, broadband sound power reduction of $6.5 \mathrm{~dB}$ was achieved. However, more significant narrowband noise is generated by the vortex shedding at the serration roots. The authors concluded even though smaller serration angles lead to better broadband noise reduction, larger angles are recommended to account for the unavoidable narrowband vortex shedding noise $[26,27]$. In addition, serration length of the sawtooth should be equal to or greater than the turbulent boundary-layer thickness, and having boundary-layer separation close to the root of the sawtooth serrations is optimum to maximize their impact.

In 2019, Chen et al. [28] presented an aeroacoustic analysis of a NACA0018 airfoil with TE serrations. Experiments were conducted in an open jet wind tunnel for serrations having variable amplitudes, variable slanting angles and a constant wavelength at a Re of 500,000 and angles of attack ranging from $0^{\circ}$ to $3^{\circ}$. The obtained data confirmed that noise reduction can be achieved in the moderate frequency range at the cost of producing narrowband tonal noise associated with vortex shedding. The aforementioned tonal noise decreases when decreasing the serration amplitude. The authors also showed that the narrowband peak can be suppressed by slanting the serration roots.

The application of owl wing features on airfoils is not a straight-forward task. Thanks to the fundamental understanding of the aeroacoustic noise generation mechanisms, researchers and engineers were able to replicate some of the flow features observed around owl wings using leading and trailing edge serrations. LE serrations were proven to be a pragmatic way to imitate the aerodynamic and aeroacoustic effects of the comb-shaped LE, by destabilizing, i.e. tripping, the BL and reducing the maximum $P_{r m s}$ on the airfoil surface. Finally, TE serrations were shown to mimic the effect of TE fringe seen on owl wings, improving mixing and increasing the streamwise coherence, at the expense of emanating a narrowband tonal peak in some cases. 


\subsubsection{Surface Finlets}

Clark et al. [29] designed surface treatments that would replicate the surface-pressure attenuating effects of the downy upper surface of owl wings, in a form suitable for application on an actual airfoil. Treatments, such as finlets and rails, were installed upstream of the TE with the objective of modifying the boundary layer turbulence before acoustic scattering at the sharp edge. Aeroacoustic wind-tunnel measurements were performed on a tripped DU96-W180 airfoil at a $\operatorname{Re}$ of 3,000,000. 14 finlet and 6 rail designs were looked into, with varying heights, spacings, thicknesses (diameters) and extension beyond the TE. These configurations provided a noise attenuation of up to $10 \mathrm{~dB}$ compared to an unmodified airfoil. Observed performance was improved when the finlets did not go past the TE. The authors concluded that the extent to which the finlets can deform and decorrelate turbulent structures in the BL is the primary driver of this mechanism.

Afshari et al. [30] investigated the effect of different types of surface treatments on surface pressure fluctuations, eddy convection velocity and spanwise length scale on a flat plate model, where the BL was tripped. The experimental results revealed that having coarser finlet spacing reduces the surface pressure power spectral density at mid to high frequency and increases the spanwise length scale, while finer spacings effectively suppress higher frequency fluctuations with a penalty in the low to mid frequency range, and a reduction in the spanwise length scale. Lastly, the finlet-spacing to boundary layer thickness ratio was found to be a critical parameter for achieving optimum surface pressure power spectral density reduction. Shi et al. [31] conducted a comprehensive trend analysis of finlets. The effects of height, spacing, thickness, position and incoming flow velocity were considered. The authors concluded that TE noise is further reduced as finlet height is increased, that finlet thickness has no significant impact on the generated noise and that small finlet spacings are more effective for high frequency noise suppression while wider spacings perform better at low frequencies. There was no 
clear trend with length variation. Afshari et al. [32] undertook a comprehensive experimental investigation using a flat plate. The authors demonstrated that finlets reduce the surface pressure fluctuation power spectral density, which can be attributed to the "channeling phenomenon" and the subsequent reorganization of the turbulent structures within the boundary layer.

In 2020, Gstrein, et al. [33] performed a parametric study comprising different placement locations and spacings for a range of conventional finlet treatments applied on a flat plate. To obtain minimal power spectral density of surface pressure fluctuations, application of the treatment further upstream the TE was favored, which allows the BL to evolve and settle rather than being scattered immediately when leaving the finlets in a disturbed state. Furthermore, the efficiency of the treatment was observed to improve by the variation of the finlet profile. Parameters like finlet spacing, height and position were varied and far-field noise was recorded. The treatment efficiency was shown to be dependent on the ratio of finlet height to BL thickness. Since the BL thickness changes with the AOA, the treatment efficiency is also a function of the airfoil AOA. It was deduced that the prevailing noise reduction mechanism in this case is the detachment of smallscale turbulence structures from the airfoil surface, which also agrees with the findings of Bodling and Sharma [34]. In conclusion, the authors think that the setting of critical configuration parameters in the case of airfoil finlets is problem dependent and is to be identified by the user accordingly. Finlets are proven to affect the flow field in a manner similar to that of the owl wing surface, which makes them an attractive TE modification for noise suppression.

Finlets are shown to be another effective means of reducing TE noise by replicating the surface effect of owl wings. On a fundamental level, finlets make use of different noise-attenuating mechanisms than serrations. 


\subsubsection{Aeroacoustic Analogies}

Given the problem-dependence of the aforementioned analyses and the high nonlinearity of fluid flow behavior, the need for practical, short response-time noise prediction methods remains crucial in the design phase. This subsection discusses the aeroacoustic analogies that laid the foundation for the development of numerical noise prediction methods.

In 1952, Lighthill [35] made an important distinction between aerodynamically generated sound and sound produced from the vibration of solids. Lighthill's contribution was a revolutionary step for the field of aeroacoustics, as he is the first to isolate aerodynamic noise, which had claimed a great deal of research at that time. Lighthill attempted to estimate the intensity of the generated sound in terms of the details of the fluid flow producing it. He deduced that the sound radiated by a fluid flow is equivalent to that which would be produced by a static distribution of acoustic quadrupoles. Physically, this means that sound is generated by fluid flow the same way as in a uniform medium being acted upon by externally applied fluctuating stresses. Following this analogy, the generated sound can be evaluated if the fluid flow is known, and complex fluid processes can be represented by equivalent acoustic sources. In his second paper in 1954, Lighthill [36] further extended his theory and made a detailed theoretical examination of the sound field produced by a turbulent jet. However, his theory was still limited to application where no solid boundaries exist in the flow, or in which the effect of such boundaries can be neglected.

In 1955, mathematician N. Curle [37] considered the part played by solid boundaries, making the analogy more complete. In a physical sense, solid boundaries make their presence felt in two ways:

i. Solid boundaries will reflect and deflect the sound generated by the quadrupoles in Lighthill's theory.

ii. The quadrupoles will only be distributed throughout the region external to the solid boundaries. 
Curle added a surface integral representing a modification to Lighthill's theory, necessitated by the presence of solid boundaries, and showed it to be equivalent to the sound generated by the distribution of dipoles representing the fluctuating forces with which the solid boundary acts on the fluid. Furthermore, a dimensional analysis was used to show that dipoles become increasingly more dominant as the flow Mach number decreases. Some years later in 1969, engineers John Ffowcs Williams and David L. Hawkings extended the Lighthill-Curle model one more time by adding a third source term to include the sound generated by arbitrarily moving surfaces [8].

As a result of the combined work of these pioneers, sound in general can be predicted by three source distributions. The first is of acoustic quadrupoles (Lighthill 1952) distributed throughout the external region surrounding the surfaces. These quadrupoles are supplemented by a surface distribution of acoustic dipoles (Curle 1955), emerging from the unsteady loading on the solid surfaces, and a surface distribution of acoustic monopoles (J. E. Ffowes Williams and D. L. Hawkings, 1969), if the surfaces are moving relative to the flow. Using these analogies, pressure fluctuations within a simulation domain can be propagated to receivers located outside that domain at a fraction of the computational cost. In other words, the aerodynamic and acoustic fields are decoupled. The Ffowcs Williams-Hawkings (FW-H) model is explained in more detail in subsection 2.4.

\subsubsection{Aerodynamic Noise Prediction}

This subsection discusses different methods that can be used to analyse and predict the noise radiated by airfoils. The majority of the methods used in industrial applications are based on semi-empirical correlations obtained by different investigators. The level of accuracy obtained by these methods is usually enough for the early phases of the design, but once the initial phase is done, more complex methods are needed to solve specific problems and gain an improved understanding of the underlying flow mechanisms. However useful this is, the corresponding 
computational power needed often makes the implementation impractical for the early design stages.

Current TE noise prediction methods include analytical, semi-empirical and numerical models. Naturally, early efforts were mainly focused on analytical solutions that quantify and predict noise radiation. Chase [38,39], Chandiramani [40], Amiet [41] and Howe [42] tackled aerodynamic sound generation as a diffraction problem, where the near field pressure fluctuations are scattered by the discontinuity at the TE. The aim was to explicitly relate the magnitude of the scattered sound in the far-field to the wall-pressure fluctuations characterizing the turbulent boundary layer (TBL). Howe's work [42] included a critical review of the theory of sound generation by the interaction of a low Mach number turbulent flow with a semi-infinite rigid plate. In another publication in 1991, Howe [43] argues that despite the increase in the airfoil physical wetted length when introducing TE serrations, the spanwise wetted length that actually contributes to noise generation is effectively reduced. Howe also concluded that optimal attenuation would be achieved when using serrations with a sawtooth profile with edges inclined at less than $45^{\circ}$ to the mean flow. Even though Howe's results are in the limit of infinitesimal mean Mach number flows, he finds it unlikely that the conclusions will significantly differ at finite Mach numbers. Amiet's model [41] simplifies the airfoil as a flat plate. Amiet used the pressure characteristics of the surface of the plate and evaluated the Kirchoff radiation integral to calculate the far-field noise. This model was later expanded by Moreau et al. to include the effect of the LE on the radiated noise, which in turn improved the low-frequency predictions [44].

Alternatively, semi-empirical models can be applied. An example includes the BPM model developed by Brooks at al. [5]. Despite being relatively practical, semi-empirical models can be of questionable accuracy when the geometry and flow parameters deviate from those in the primary investigation. Recent advancements include the coupling of such models with steady-state ReynoldsAveraged Navier-Stokes (RANS) simulations. Mayer et al. [45] used the mathematical model developed by Lyu et al. [46] to create a semi-analytical model that is fed boundary layer parameters from steady-state RANS CFD simulations. 
The objective was to estimate the noise reduction performance of serrated airfoils. It was shown that different boundary layer (BL) extraction locations significantly influence the predicted noise reduction, which indicates that the proposed method is not mature yet. In 2018, Faßmann et al. [47] used a similar approach to predict broadband TE noise. A RANS simulation was used to provide the time-averaged solution of a turbulent flow, and fluctuating sound sources were generated with the Fast-Random Particle-Mesh Method to then stochastically generate sound sources and simulate the broadband sound generation. The method was also extended to include the effects of porous materials near the trailing edge. Shi el al. used RANS simulations to provide BL parameters near the airfoil TE as input for an empirical wall pressure spectrum model [48]. The output pressure fluctuations were then used to predict the far-field noise is using Howe's diffraction model [49].

Chen et al. [50] presented a parametric airfoil design tool that combines the same empirical wall-pressure spectrum model developed by Lee [48] and Howe's diffraction model [49] to analyse the effects of different design parameters on airfoil TE noise. It was found that parameters like the airfoil camber, thickness and TE camber significantly affect the generated noise. More sophisticated methods are still needed to terminate, or significantly reduce, the dependence of the output solution on statistical information, and provide turbulent flow information to be coupled with the aeroacoustic predictions. In 2020, Cao et al. [51] proposed a novel modeling method to replicate the aerodynamic and aeroacoustic features of TE serrations. The method consists of substituting extra lift and drag sources into the momentum equation of the flow around a baseline airfoil. One of the interesting observations made when inspecting the flow field was the existence of a pair of counter rotating vortices induced by the spanwise pressure gradient of the serrations.

Computational aeroacoustics (CAA) simulations simultaneously calculate the hydrodynamic and acoustic fields. This can be done using direct numerical simulations (DNS) [52] or using high fidelity flow simulations coupled with acoustic noise propagation models [31,53-55]. Since noise is nothing but pressure fluctuations that reach the observer, explicitly solving the governing equations of 
fluid flow is the most accurate numerical approach available. CAA approaches are often used in the design stage to gain a better understanding of the physics of noise generation. An alternative approach is using Large Eddy Simulations (LES), which are cheaper than DNS but still considerably costly.

In 2000, Manoha, Troff and Sagaut [56] successfully predicted the far-field noise generated by turbulence flowing over the blunt trailing edge of a thick flat plate by coupling a LES with Curle's solution to the Lighthill equation, then the Ffowcs-Williams and Hawkings (FW-H) analogy. Agrawal and Sharma [57] used LES to simulate the interaction between the wake of a cylindrical rod and a downstream airfoil. Tang et al. [58] performed simulations employing LES and the Lighthill-Curle method in an attempt to simulate the hydrodynamic field and sound sources associated with TE serrations on a NACA0012 airfoil. It was confirmed that TE serrations reduce the radiated noise, given that serrations impede the growth of spanwise vortices, i.e. decrease spanwise coherence, and promote streamwise ones near the wake. It was also observed that the velocity fluctuations perpendicular to the mainstream decrease, resulting in weaker pressure fluctuations near the TE. Lastly, it was confirmed that applying serrations upstream of boundary layer reattachment is effective at reducing the strength of the sound source and subsequently the generated noise [58].

Bodling et al. [34] presented a numerical investigation of bio-inspired airfoils finlets. The used geometries were characterized by an array of finlets applied near the TE and aligned with the flow. LES simulations were carried out on a NACA0012 airfoil for two different fence heights. Results showed a far-field SPL reduction for the frequency range $500 \mathrm{~Hz}-5 \mathrm{kHz}$ using shorter fence, while the longer one's impact on the radiated noise is limited to frequencies higher than $1.5 \mathrm{kHz}$. Above $2 \mathrm{kHz}$, greater noise reduction is observed with the taller fence. Finlets were shown to lift the turbulent eddies away from the scattering TE, thereby increasing the source-scattering edge distance. This mechanism decreases the noise scattering efficiency and the far-field noise produced.

Zilstra and Johnson [59] demonstrated the ability of LES, combined with the FW-H acoustic analogy, to predict the flow field and acoustic results for a 
SD7037 airfoil at a Re of 43,000 and different angles of attack. The method was proven to be an effective airfoil self-noise prediction tool at static AOA. In addition, the zero-degree AOA model successfully predicted two narrowband peaks corresponding to $2 \mathrm{D}$ vortex shedding and the flow transition from $2 \mathrm{D}$ to $3 \mathrm{D}$ boundary layer behavior.

LES remains prohibitively expensive and impractical in most industrial applications. To address the pertinent need for reliable, short-response-time noise prediction methods, several researchers have attempted to develop new, suitable simulation techniques that would notably decrease the required computational effort. In 2002, Quéméré and Sagaut [60] presented a novel zonal multi-domain RANS/LES method (also known as Embedded LES or ELES), where the full domain configuration was decomposed into several subdomains that can be treated with either RANS or LES. The same concept was later adopted by Teraccol [61], who investigated using ELES to represent aerodynamic noise sources. The method was applied to a flat plate with a blunt trailing edge and a NACA0012 airfoil. In this approach, zonal LES is only performed close to the main elements responsible for sound generation, while the overall configuration is treated by a RANS. The most critical point was the numerical treatment performed at the inlet of the LES domain, where two main approaches were tested and implemented: (1) the use of a recycling strategy, and (2) synthetic coherent structures generation through an analytical model. CPU time reductions in the order of 40 were obtained and the method was found to be an attractive compromise between accuracy and computational cost. In 2008, Fröhlich and von Terzi [62] presented a generic review of the various hybrid RANS/LES approaches along with different interface treatment strategies. The review also provided information on how to distinguish between different hybrid methods. Successful simulation results demonstrated the high potential of the approach.

Mathey [63] evaluated the use of LES for the prediction of broadband and tonal noise generated by the flow past an airfoil trailing edge at a high Reynold's number (Re). Two simulations were performed for a free stream velocity of 30.5 $\mathrm{m} / \mathrm{s}$ and a chord based Re of $1,800,000$. The first one used a random forcing method 
at the RANS/LES interface, and the second one used the Vortex Method (VM). The far-field noise was calculated using the FW-H model. The results showed that the technique is capable of capturing the separated flow and reproducing the main characteristics of the aeroacoustic sources. Lastly, it was shown that the use of the VM for the generation of a synthetically turbulent flow field significantly improved the accuracy of the simulation.

Kim et at. [64] used ELES approach to predict the aeroacoustic and aerodynamic properties of different flatback airfoils at high Re and compared the results to semi-empirical and experimental data. Synthetic turbulence was generated at the RANS/LES interface using the VM, and far-field acoustics were computed using the FW-H analogy. ELES successfully predicts a tonal peak frequency of $150 \mathrm{~Hz}$ and experimental results show a peak at $200 \mathrm{~Hz}$. The hybrid RANS-LES method was found to be adequate for predicting aerodynamic noise generation by vortical flow in the vicinity of a blunt TE airfoil. Lane, Croaker and Ding [65] tested and implemented ELES for the prediction of TE noise due to flow around a NACA0012 airfoil. The obtained results were compared to a full LES simulation and to experimental data. Both simulations used the same mesh resolution and the same wall-modeled LES approach. For ELES, the mesh size was only about 13 million cells, compared to 40 million cells for the full LES. It was found that the results of both simulations were in good agreement. The ELES approach resulted in an impressive saving of $55 \%$ of the computational cost of a full LES.

Zuo et al. [66] performed flow simulations using ELES to analyse the aerodynamic and noise characteristics of a serrated-TE NACA0018 airfoil at a Re of 160,00 and an angle of attack of $6^{\circ}$. Two airfoils having the same serration wave length and different amplitudes were considered and compared to a straight TE case. Predictions based on the FW-H acoustic analogy showed that longer serrations are more effective in decreasing the overall sound pressure levels. Longer sawtooth serrations were also shown to reduce the amplitudes of pressure fluctuations near the airfoil surface. 
In 2021, Romani et al. [67] investigated the flow field around a NACA64618 airfoil at different angles of attack and serration flap-angles. The numerical flow solution was obtained by solving the fully explicit lattice Boltzmann equation and the generated aerodynamic noise was estimated using a model based on Farassat's formulation of the FW-H analogy [68]. It was observed that most of the noise in the case of a serrated TE is generated near the serration roots. It's worth noting that the simulations were run on an Intel Xeon E5-2690 2.90 GHz platform of 360 cores, where the CPU runtime per flow passage for different mesh resolutions is up to 2100 hours and 15600 hours.

To summarize, numerous techniques were found suitable for the prediction of far-field noise. High-fidelity techniques like DNS or LES can be fundamentally more accurate, but the increased accuracy comes with a substantial surge in computational cost. On the other hand, far-field noise can be inexpensively predicted from low cost statistical and semi-empirical models. The first drawback of these models is the dependence of the output solution on statistical data, which is a significant constraint when researchers are working on innovative designs for which no recorded data is available. Furthermore, such models cannot provide the information needed to visualize and understand the underlying physics of the noisegenerating flow. In between the extremes lies segregated modeling, which has acquired a great deal of attention as it constitutes a potentially good compromise between accuracy and cost. It will always be up to the user to choose the most convenient approach, depending on the flow parameters of interest, required accuracy and the available computational resources. 


\subsubsection{Objectives of the Thesis}

This thesis aims to utilize ELES and experimental wind tunnel testing to investigate the fundamental noise attenuating mechanisms of different bio-inspired airfoil TE designs. More specifically, efforts include:

1. Development of a working ELES framework to be coupled with the FW-H aeroacoustic analogy, which allows for the extraction of accurate turbulent flow data and providing far-field noise predictions by leveraging aeroacoustic analogies, thus eliminating the need for full domain resolution.

2. Demonstration of mesh convergence, and comparison of computational results with experimental data.

3. Application of the proposed method to simulate the flow around different noise attenuating TE designs, such as standard serrations and finlets, providing an understanding of the underlying flow mechanisms using contours of turbulent statistics obtained from the transient numerical simulations.

4. Investigation of the effect of different standard serration parameters on the radiated tonal noise.

5. Examination of finned serrations, a new TE design developed with the purpose of superimposing the noise reducing effects of finlets and standard serrations.

6. Evaluation of the use of slanted-root serrations for tonal peak suppression, and analysis of their effect on the airfoil wake.

7. Experimental investigation of the effect of airfoil AOA on noise reduction in TE finlets, relative to a straight TE airfoil. 


\subsubsection{Research Contributions}

The research contributions presented in this thesis are summarized in this section.

1. (Chapter 3 \& Chapter 4) Successful implementation of ELES in tandem with the FW-H aeroacoustic analogy to predict flow-field around NACA0012 airfoils equipped with different bio-inspired noise-attenuating TE designs. Far-field noise is also estimated at a fraction of the computational cost needed for full LES or DNS resolution.

2. (Chapter 3 \& Chapter 4) Replication of different owl wing features on NACA0012 airfoils using practical designs, such as serrations to mimic the effect of the TE fringe, and finlets to mimic that of the downy upper surface.

3. (Chapter 4) Prediction of the fundamental flow characteristics over and past serrated TE airfoils and bio-inspired TE designs.

4. (Chapter 4) Establishment of a relationship between the flow aerodynamic mechanisms and the radiated far-field noise.

5. (Chapter 4) Successful suppression of the tonal peaks associated with standard serrations using slanted-root serrations, and interpretation of their effect on wake vortex shedding.

6. (Chapter $2 \&$ Chapter 4) Demonstration of the noise reduction dependence on airfoil AOA for TE designs using finlets, and subsequently its dependence on the BL thickness. The obtained overall noise reduction is shown to increase as the airfoil AOA is increased.

The work from this thesis has resulted in the following publications:

1. Salama, Y., and Rocha, J. (2021, August). ELES and Tonal Noise Prediction of Slanted-Root Trailing-Edge Serrations. Conference: 50th International Congress and Exposition on Noise Control Engineering (InterNoise 2021, Washington, DC, USA.

2. Salama, Y., Al Tlua, B., and Rocha, J. (2021). ELES and Tonal Noise Prediction of Serrated-TE Airfoils. MDPI Aerospace (Under Review). 
3. Salama, Y., and Rocha, J. (2021). ELES and Experimental Testing of TE Finlets (In Preparation).

\subsubsection{Structure of the Thesis}

Chapter 1 provides a detailed literature review on TE noise reduction and prediction. Aeroacoustic noise generation is discussed, with a focus on TE noise. The morphological features of owl wings used in biomimicry are also shown and explained. The history of aeroacoustic analogies is summarized.

Chapter 2 presents the numerical methods employed in ELES, in terms of governing equations, ELES interface treatment and aeroacoustic noise propagation.

Chapter 3 describes the wind tunnel facility used for experimental testing, providing details on the chosen airfoil TE design and its fabrication, the wind tunnel setup and experimental sampling techniques.

Chapter 4 provides details about the ELES setup, the computational mesh characteristics, the process followed for each of the simulated TE designs and the necessary flow information and boundary conditions.

Chapter 5 presents the results obtained from ELES and experimental testing. Surface pressure statistics, turbulence kinetic energy distribution, vorticity magnitude, Q-Criterion iso-surfaces and far-field noise spectra are presented and discussed.

Conclusion and recommendations for future work are provided in

\section{Chapter 6.}




\section{Chapter 2}

\section{Numerical Methods}

This chapter explains the numerical methods used in ELES, including: the governing equations, LES, RANS/LES interface treatment and the FW-H aeroacoustic analogy. In this study, the fluid flow is solved using a numerical solution of the Navier-Stokes (NS) equations. The entire fluid domain is broken up into small elements, then equations are discretized and iteratively solved using the ANSYS Fluent commercial software. Fluent follows an element based Finite Volume Method (FVM), where the spatial fluid domain is split into a finite set of control volumes, over which the conservation equations are solved. Seeing that most of these equations are non-linear partial differential equations, they are discretized into a system of algebraic equations then numerically solved to render a solution. Subsequently, the approximate value of each variable at specific points throughout the domain is calculated. Subsection 2.1 provides the general governing equations of fluid flow, i.e. the NS equations, 2.2 focuses on LES and turbulence resolution, 2.3 discusses the RANS/LES treatment and 2.4 presents the FW-H aeroacoustic model.

\subsection{Governing Equations}

The main equations needed to fully describe a fluid in motion are the conservation of mass, also known as the continuity equation, and the conservation of momentum. The continuity equation is simply a scalar equation that reflects the fact the mass is a conserved property in any continuum mechanics analysis, such that 


$$
\frac{\partial \rho}{\partial t}+\nabla \cdot(\rho \boldsymbol{v})=0
$$

where $\rho$ is the fluid density, $v$ is the fluid velocity and $t$ is time. On the other hand, the conservation of momentum equation (equation 2) is a set of vector equations that can be expanded depending on the coordinate system in use. When broken down, they simply state that " $\mathrm{F}=$ ma", which is Newton's second law of motion.

$$
\rho\left(\frac{\partial \boldsymbol{v}}{\partial t}+\boldsymbol{v} \cdot \nabla \boldsymbol{v}\right)=-\nabla P+\mu \nabla^{2} \boldsymbol{v}+\rho \boldsymbol{f}
$$

$P$ is the fluid pressure, $\mu$ is the kinematic viscosity and $\boldsymbol{f}$ represents the body forces acting on the fluid. Together, these equations are termed the Navier-Stoke equations. In principal, these equations suffice to describe both laminar and turbulent flows without the need for any additional information. However, the DNS of these flows requires extraordinary computing power, many orders of magnitude higher than available in the near future. This is due to the large span of turbulent length and time scales that exist in a turbulent flow. As the flow Re increases, the computational power required also increases.

To overcome this problem, statistical turbulence models were developed to approximate the effects of turbulence without recourse to DNS. The original unsteady NS equations are modified to only represent the mean flow quantities. The resulting equations are called the RANS equations. RANS equations greatly reduce the computational effort compared to DNS. Subsequently, equations (1) and (2) can be expanded for cartesian coordinates to become

$$
\begin{gathered}
\frac{\partial \rho}{\partial t}+\frac{\partial}{\partial x_{j}}\left(\rho U_{j}\right)=0 \\
\frac{\partial\left(\rho U_{i}\right)}{\partial t}+\frac{\partial}{\partial x_{j}}\left(\rho U_{i} U_{j}\right)=-\frac{\partial P}{\partial x_{i}}+\frac{\partial}{\partial x_{j}}\left(\tau_{i j}-\rho \overline{u_{i} u_{j}}\right)
\end{gathered}
$$

where $\tau_{i j}$ is the molecular stress tensor. The continuity equation remains unaltered but the momentum equations contain a new turbulent flux component, $\rho \overline{u_{i} u_{j}}$. This term a property of the flow, not the fluid, and represents the so-called Reynold's stresses. Then, following the eddy viscosity hypothesis [69], the Reynolds stresses 
can be related to the mean velocity gradients with the introduction of a new term, $\mu_{t}$, called the eddy or turbulent viscosity. The most popular two equation turbulence models, $k-\epsilon$ and $k-\omega$, use this variable. Following this procedure, equations (4) becomes

$$
\frac{\partial\left(\rho U_{i}\right)}{\partial t}+\frac{\partial}{\partial x_{j}}\left(\rho U_{i} U_{j}\right)=-\frac{\partial P^{\prime}}{\partial x_{i}}+\frac{\partial}{\partial x_{j}}\left(\mu_{e f f}\left(\frac{\partial U_{i}}{\partial x_{j}}-\frac{\partial U_{j}}{\partial x_{i}}\right)\right)
$$

where $\mu_{e f f}=\mu+\mu_{t}$ and $P^{\prime}$ is the modified pressure.

The $k-\epsilon$ turbulence model introduces two new variables to which the turbulent viscosity is linked through equation (6). $k$ is the turbulence kinetic energy and is defined as the change in the velocity fluctuations, having the units $L^{2} T^{-2}$. The second term, $\epsilon$, is the turbulence dissipation rate. It is an indication of the rate at which velocity fluctuations are dissipated and has the units $L^{2} T^{-3}$.

$$
\left.\mu_{t}=C_{\mu} \rho \frac{k^{2}}{\epsilon} \frac{\partial U_{j}}{\partial x_{i}}\right)
$$

The values of $k$ and $\epsilon$ are obtained directly by solving differential transport equations for the turbulence kinetic energy and turbulence dissipation rate $[70,71]$. It is important to note that production limiters, which are damping functions, can also be defined to avoid the build-up of kinetic energy near stagnation regions. This model is generally suitable when predicting flow far from the boundaries.

On the other hand, he $k-\omega$ model [72] does not include the non-linear damping functions used in the $k-\epsilon$ model. It is therefore more accurate and more robust, having the advantage of near wall treatment for low Reynold's Number problems. It relates the kinetic energy and turbulence frequency to the turbulence viscosity through equation (7).

$$
\mu_{t}=\rho \frac{k}{\omega} \frac{\partial U_{j}}{\partial x_{i}}
$$

where the variable $\omega$ is the specific dissipation, which dictates the scale of the turbulence, while $k$ determines the energy contained in the turbulence. 
In 1994, Menter [73] developed the Shear Stress Transport (SST) model, which is fundamentally based on the $k-\omega$ model but combines the advantages of $k-\omega$ and $k-\epsilon$. Two new blending functions, $F_{1}$ and $F_{2}$, are introduced. The first one allows the switching between the $k-\varepsilon$ and $k-\omega$ models depending on how far each cell is from the closest wall. When $F_{1}=0$, the $k-\varepsilon$ equation is solved, thus avoiding the $k-\omega$ oversensitivity to inlet free-stream turbulence properties. When $F_{1}=1$, the $k-\omega$ formulation is used. $F_{2}$ is called the viscosity limiter and is useful to avoid wall shear overprediction by reducing the turbulent viscosity near the walls. $F_{1}$ and $F_{2}$ are scalar fields that take values between zero and 1 for every cell in the mesh. Thanks to aforementioned blending functions, the SST model has the added advantage accounting for the transport of turbulent shear stresses, thus giving accurate predictions of flow separation under adverse pressure gradients [73].

The SST model is chosen to initialize the flow in the presented simulations, and the simplified equations are iteratively solved using the commercial software ANSYS Fluent. Although very useful, the discussed models are not sufficient for the prediction of radiated noise, as they yield time-averaged (steady-state) solutions. Given the fluctuating nature of noise, transient simulations are needed. The initialized flow provides an initial guess for the necessary transient models, which are discussed in subsection 2.2. The initialization stage is also crucial to convey correct statistical turbulence information to the RANS/LES interface discussed in subsection 2.3.

\subsection{Large Eddy Simulation (LES)}

After flow initialization, the next step is to compute the hydrodynamic field using a time-dependent model, LES [74]. LES was originally proposed for the simulation of atmospheric flows and has since become one of the most powerful tools used to simulate turbulent flows [75]. 
In this method, only the large energy-containing eddies are resolved. This is based on the observation that larger eddies are problem dependent. They contain most of the energy and momentum, while the smaller eddies behave in a somewhat universal way, for which the chances of finding a turbulence model are much higher $[76,77]$. This approach allows a much coarser mesh than DNS and provides a good compromise between accuracy and computational cost. The governing equations, termed the spatially-filtered NS equations, are obtained by applying a low pass filter on the time dependent NS equations in the physical space, and the flow is assumed incompressible. In order to increase efficiency, the filter width is the same size as the mesh spacing used in the computational domain. The resulting equations describe the dynamics of large eddies [76,77]. Field variables, such as pressure and velocity, are defined by their convolution with a filter function over the fluid domain:

$$
\bar{\phi}(x)=\int_{D} \phi\left(x^{\prime}\right) G\left(x, x^{\prime}\right) d x^{\prime}
$$

where $\mathrm{D}$ is the fluid domain and $\mathrm{G}$ is the filtering function. The overbar indicates spatial filtering and not temporal averaging. After implicitly applying the filter to the mass and momentum conservation equations using the computational mesh, the governing equations become:

$$
\begin{gathered}
\frac{\partial \rho}{\partial t}+\frac{\partial}{\partial x_{i}}\left(\rho \bar{u}_{i}\right)=0 \\
\frac{\partial}{\partial t}\left(\rho \bar{u}_{i}\right)+\frac{\partial}{\partial x_{j}}\left(\rho \bar{u}_{i} \bar{u}_{j}\right)=\frac{\partial}{\partial x_{j}}\left(\sigma_{i j}\right)-\frac{\partial \bar{p}}{\partial x_{i}}-\frac{\partial \tau_{i j}}{\partial x_{j}}
\end{gathered}
$$

where $\sigma_{i j}$ is the molecular viscosity stress tensor, while $\tau_{i j}$ represents the subgridscale stresses (SGS), and is defined as:

$$
\tau_{i j}=\rho \overline{u_{\imath} u_{\jmath}}-\rho \bar{u}_{i} \bar{u}_{j}
$$


In order to obtain a closed system of equations, the unknown SGS stresses are modeled by applying the Boussinesq eddy viscosity hypothesis [69], thus computing the subgrid-scale turbulent stresses from

$$
\tau_{i j}-\frac{1}{3} \tau_{k k} \delta_{i j}=-2 \mu_{t} \bar{S}_{i j}
$$

where $\mu_{t}$ is the SGS turbulent viscosity and $\tau_{k k}$ is the isotropic part of the SGS stress tensor. The latter part is not modeled as it is added to the filtered static pressure term. $S_{i j}$ is the strain-rate tensor of the resolved scale calculated from equation (13) using the filtered velocity components.

$$
\bar{S}_{i j}=\frac{1}{2}\left(\frac{\partial \overline{u_{l}}}{\partial x_{j}}+\frac{\partial \bar{u}_{j}}{\partial x_{i}}\right)
$$

For the Wall Adapting Local Eddy Viscosity (WALE) model [78], $\mu_{t}$ is modeled as:

$$
\mu_{t}=\rho L_{s}^{2} \frac{\left(S_{i j}^{d} S_{i j}^{d}\right)^{\frac{3}{2}}}{\left(\overline{S_{l j}} \overline{S_{l j}}\right)^{\frac{5}{2}}\left(S_{i j}^{d} S_{i j}^{d}\right)^{\frac{5}{4}}}
$$

$L_{s}$, the mixing length of the sub-grid scale, and $S_{i j}^{d}$, which is a function of the strain and rotation rate tensors, are defined in equations (15) and (16) as

$$
\begin{gathered}
L_{s}=\min \left(\kappa d, C_{w} V^{\frac{1}{3}}\right) \\
S_{i j}^{d}=\frac{1}{2}\left(\bar{g}_{i j}^{2}+\bar{g}_{j i}^{2}\right)-\frac{1}{3} \delta_{i j} \bar{g}_{k k}^{2}
\end{gathered}
$$

And $g_{i j}$ is defined in equation (17) as

$$
\bar{g}_{i j}=\frac{\partial \bar{u}_{i}}{\partial x_{j}}
$$

$\mathrm{d}$ is the distance to the closest wall, $\mathrm{V}$ is the volume of the computational cell, $\kappa=0.41$ is the von Kármán constant and $C_{w}=0.325$ is the WALE constant.

It is relatively cheaper to resolve time-dependant flow quantities using LES than DNS. However, LES is still prohibitively time-consuming, and limited to 
certain applications. In order to address the need for reliable short-response-time noise prediction methods for industrial application, segregated modeling is employed. The next subsection presents the concept of ELES and discusses the RANS/LES interface treatment.

\subsection{ELES and RANS/LES Interface Treatment}

In the presented approach, the entire flow domain is decomposed into clearly identifiable regions for RANS and LES before the simulation is started. This is usually referred to as segregated modeling. The goal is to use each model where it is best suited. The RANS equations provide stationary field statistics and LES resolves the unsteady high-resolution perturbations where it is needed.

The used technique is termed an Embedded Large Eddy Simulation (ELES). The main encountered difficulty is defining proper interface conditions, seeing that inappropriate coupling could lead to results contamination in the LES or RANS subdomains. At the inflow interface, mass, momentum and energy are convected into the LES subdomain from the RANS region. The latter provides mean values which are to be coupled with the LES equations. To obtain correct LES results, fluctuations must be provided at the interface and added to the mean flow computed by RANS. The fluctuations can be real, provided by precursor simulations or databases of similar flows, or synthetic, provided by Fourrier modes, digital filters, random vortices...etc. The goal is to make the imposed fluctuation as close as possible to those present in a real physical flow.

The VM [79] is chosen as a means of adding artificial resolved turbulence at the RANS/LES interface. In this approach, a fluctuating vorticity field is added to the mean flow, consequently creating perturbations similar in behavior to realistic ones. The VM is based on the Biot-Savart law and the 2D evolution equation of vorticity. Vortex points, or particles, are distributed over the inlet interface perpendicular to the streamwise direction and are randomly convected, carrying information about the vorticity field. The amount of vorticity carried by a 
given particle "i" is represented by the circulation, $\Gamma$, according to equation (18), and the assumed spatial distribution is given by equation (19).

$$
\begin{aligned}
\Gamma_{i}(x, y) & =4 \sqrt{\left(\frac{\pi A k}{3 N(2 \ln (3)-3 \ln (2))}\right)} \\
\eta(\vec{x}) & =\frac{1}{2 \pi \sigma^{2}}\left(2 e^{\frac{-|x|^{2}}{2 \sigma^{2}}}-1\right) 2 e^{\frac{-|x|^{2}}{2 \sigma^{2}}}
\end{aligned}
$$

$N$ is the number of vortex points, $A$ is the inlet section area, $k$ is the turbulence kinetic energy and $\sigma$ controls the size of the vortex particles. The resulting discretization for the velocity field is given by

$$
\vec{u}(\vec{x})=\frac{1}{2 \pi} \sum_{i=1}^{N} \Gamma_{i} \frac{\left(\left(\overrightarrow{x_{l}}-\vec{x}\right) \times \vec{z}\right)}{\left|\vec{x}-\vec{x}_{l}^{\prime}\right|^{2}}\left(1-e^{\frac{\left|\vec{x}-\vec{x}^{\prime}\right|^{2}}{2 \sigma^{2}}}\right) e^{-\frac{\left|\vec{x}-\vec{x}^{\prime}\right|^{2}}{2 \sigma^{2}}}
$$

where $\vec{z}$ is a unit vector in the streamwise direction and $x_{i}$ is the location of the i-th vortex particle. The value of $\sigma$ is calculated from a known profile of mean turbulence kinetic energy and mean dissipation rate at the inlet, such that

$$
\sigma=\frac{c k^{3 / 2}}{2 \varepsilon}
$$

where $c=0.16$. The minimum value of $\sigma$ is determined by the local mesh size to ensure that the vortices will always belong to the resolved scale. Furthermore, the sign of the circulation of each vortex is randomly changed every characteristic time scale, which is the time needed for a $2 \mathrm{D}$ vortex to travel $n$ times its mean characteristic 2D size in the boundary normal direction, where $n$ is set to equal 100 from numerical testing. Finally, a rescaling model is used, and the velocity fluctuations are expressed as 


$$
u_{i}^{\prime *}=u_{i}^{\prime} \frac{\sqrt{<u_{i} u_{i}>}}{\sqrt{\frac{2}{3 k}}}
$$

where $u_{i}{ }^{*}$ and $u_{i}{ }^{\prime}$ are the scaled and unscaled velocity fluctuations, and $<u_{i} u_{i}>$ represents the normal statistic velocity fluctuations.

In summary, the simulation domain is decomposed into 2 regions; RANS and LES. The VM is used to inject artificial turbulence to the transient domain based on the solution of the steady-state one. Following the resolution of the flow field in the vicinity of the airfoil, pressure fluctuations need to be resolved at the location of a set receiver to obtain the far-field noise spectrum. Since receivers are often meters away from the airfoil, expanding the simulation domain to include the receiver is impractical and computationally expensive. The following subsection discusses the aeroacoustic analogies used to overcome this challenge.

\subsection{The Ffowes Williams and Hawkings (FW-H) Aeroacoustic Analogy}

In 1952, Lighthill was the first researcher to distinguish between aerodynamically generated sound and sound produced from the vibration of solids. Lighthill's work is revolutionary in the sense that it allowed for the estimation of the intensity of generated sound in terms of the details of the fluid flow producing it. Using Lighthill's acoustic analogy $[35,36]$, the near-field flow is computed using the appropriate flow-governing equations, and the far-field noise can be predicted with the aid of an analytically derived integral solution to the wave equation. The acoustic analogy decouples sound generation from its propagation, thus allowing the separation of the flow solution from the acoustic analysis. In terms of numerical simulations, acoustic sources can be extracted from the CFD domain, and the flow domain no longer needs to be expanded to include the receivers.

The Ffowcs Williams and Hawkings (FW-H) formulation [8] adopts the most general form of Lighthill's acoustic analogy. The FW-H equation is an 
inhomogeneous wave equation derived by manipulating the continuity and NS equations. The FW-H equation can be expressed as

in which

$$
\begin{gathered}
\frac{1}{a_{\infty}^{2}} \frac{\partial^{2} p^{\prime}}{\partial t^{2}}-\nabla^{2} p^{\prime}=\frac{\partial}{\partial t}\left\{\left[\rho_{\infty} v_{n}+\rho\left(u_{n}-v_{n}\right)\right] \delta(f)\right\}-\frac{\partial}{\partial x_{i}}\left\{\left[P_{i j} n_{j}+\right.\right. \\
\left.\left.\rho u_{i}\left(u_{n}-v_{n}\right)\right] \delta(f)\right\}+\frac{\partial^{2}}{\partial x_{i} \partial x_{j}}\left\{T_{i j} H(f)\right\}
\end{gathered}
$$

$$
T_{i j}=\rho u_{i} u_{j}+P_{i j}-a_{\infty}^{2}\left(\rho-\rho_{\infty}\right) \delta_{i j}
$$

where $p^{\prime}=p-p_{0}$ is the sound pressure at the far-field, $u_{i}$ is the fluid velocity component in the $x_{i}$ direction, $u_{n}$ is the velocity component normal to the surface, $v_{i}$ is the surface velocity component in the $x_{i}$ direction, $v_{n}$ is the surface velocity component normal to the surface, $\delta(f)$ is the Dirac delta function and $H(f)$ is the Heaviside function. The subscript " $\infty$ " denotes free-stream parameters. The $f=0$ surface is a mathematical surface representing the source surface. $n_{i}$ is a unit vector normal pointing towards the exterior region of the source $(f>0), a_{\infty}$ is the speed of the sound at the far field, $T_{i j}$ is the Lighthill stress tensor defined in equation (23) and $P_{i j}$ is the compressive stress tensor.

The first term on the RHS of equation (23) represents the monopole or thickness source, modeling the sound generated by the displacement of a fluid as a body passes through it (example: fluid injection or fluctuating fluid volumes). The second term is the dipole or loading source, resulting from the unsteadiness of the forces acting on the body's surface. The third term is the quadrupole source term, representing the non-linear fluctuations in the local sound speed and fluid velocity near the body surface (example: turbulence and fluctuating shear of fluid particles). Monopole and dipole sources are dominant in low Mach number flows. By integrating equation (23) assuming free-space flow and no obstacles between the sound source and receiver, a full solution consisting of surface and volume integrals is obtained. In the present case, the volume integral is neglected as it is only significant in high Mach number flows. Thus, the far-field sound pressure can be expressed as 
in which

$$
p^{\prime}(\vec{x}, t)=p_{T}^{\prime}(\vec{x}, t)+p_{L}^{\prime}(\vec{x}, t)
$$

$$
\begin{gathered}
4 \pi p_{T}^{\prime}(x, t)=\int_{f=0}\left[\frac{\rho_{\infty}\left(\dot{U}_{n}+U_{\dot{n}}\right)}{r\left(1-M_{r}\right)^{2}}\right] d S \\
+\int_{f=0}\left[\frac{\rho_{\infty} U_{n}\left\{r \dot{M}_{r}+a_{\infty}\left(M_{r}-M^{2}\right)\right\}}{r^{2}\left(1-M_{r}\right)^{3}}\right] d S \\
4 \pi p_{L}^{\prime}(x, t)=\frac{1}{a_{\infty}} \int_{f=0}\left[\frac{\dot{L_{r}}}{r\left(1-M_{r}\right)^{2}}\right] d S+\int_{f=0}\left[\frac{L_{r}-L_{M}}{r^{2}\left(1-M_{r}\right)^{2}}\right] d S \\
+\frac{1}{a_{\infty}} \int_{f=0}\left[\frac{L_{r}\left\{r \dot{M}_{r}+a_{0}\left(M_{r}-M^{2}\right)\right\}}{r^{2}\left(1-M_{r}\right)^{3}}\right] d S \\
U_{i}=v_{i}+\frac{\rho}{\rho_{\infty}}\left(u_{i}-v_{i}\right) \\
L_{i}=P_{i j} \widehat{n}_{j}+\rho u_{i}\left(u_{n}-v_{n}\right)
\end{gathered}
$$

A dot over a variable indicates the source-time derivative of that variable, while the subscripts " $n$ ", " $r$ " and " $M$ " denote the dot product with the unit normal vector, the unit radiation vector and surface velocity vector normalized by the speed of sound, respectively.

Using the FW-H aeroacoustic analogy, noise generation can be decoupled from its propagation and pressure fluctuations can be propagated from the CFD domain to a receiver placed outside of it at a fraction of the computational cost, which is favorable in many fields and applications as it allows for faster simulations while maintaining satisfactory accuracy. 


\section{Chapter 3}

\section{Experimental Setup and Techniques}

This subsection describes the experimental facility in which the wind tunnel tests were performed, including: a description of the Carleton University medium speed wind tunnel, the experimentally tested TE design, and the flow setup. Information on data acquisition and post-processing is also provided.

\subsection{Experimental Facility}

Experimental testing was carried out in the medium-speed, closed-loop wind tunnel facility at Carleton University, seen in Figure 3. The airflow is supplied by an axial propeller with an outer diameter of $1.2 \mathrm{~m}$, which is driven by a $37.3 \mathrm{~kW}$ (50 HP) DC motor that operates at speeds up to speeds of 900 RPM. A variable frequency drive (VFD) is used to modulate the rotational frequency of the fan at a resolution of $1.0 \mathrm{~Hz}$. It was determined that the VFD can control the flow speed in increments of $0.9 \mathrm{~m} / \mathrm{s}$, up to a maximum speed of approximately $45 \mathrm{~m} / \mathrm{s}$ [80]. A series of turbulence grids precede a 9:1 contraction, which reduces the turbulence intensity levels in the middle of the test section to less than $0.27 \%$. 


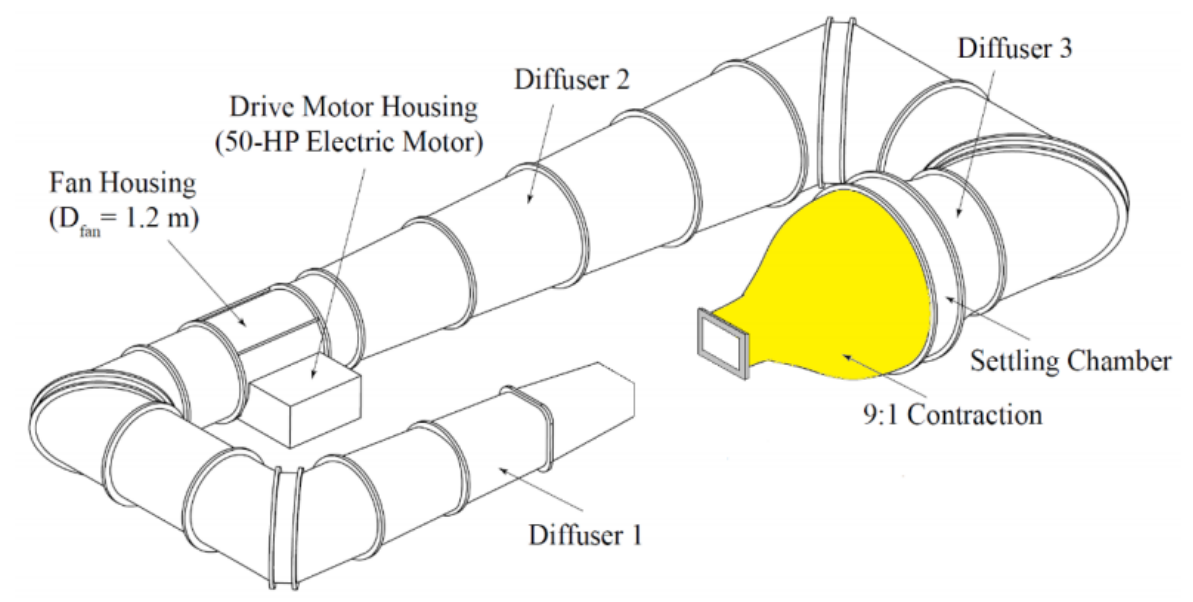

Figure 3: Carleton University medium speed wind tunnel configuration.

The wind tunnel has a removable, $0.78 \mathrm{~m}$ by $0.51 \mathrm{~m}$ rectangular test section, $1.83 \mathrm{~m}$ long. The test section is surrounded by anechoic chambers on both sides to make it useful for aeroacoustic testing (Figure 4). Both sides of the test section walls are made of stretched, thin-weave cloth extending for $1.83 \mathrm{~m}$ in the streamwise direction, which allows for a smooth flow surface. The cloth windows also allow sound to pass through the walls and into the anechoic chambers with minimum attenuation. The chambers and the rectangular test section are joined together using clamps and bolt to enforce a pressure seal and eliminate leakage. The acoustic chambers have different depths of $1.2 \mathrm{~m}$ and $0.8 \mathrm{~m}$, respectively, for the left and right sides. Both chambers have the same streamside length of $1.83 \mathrm{~m}$. The chambers are also lined with $0.015 \mathrm{~m}$ thick carpet bed, and $0.05 \mathrm{~m}$ acoustic wedges made of foam. Thanks to the absorptivity of foam and the shape of the wedges, the anechoic chambers have reduced reflection (echoes) from their walls, thus simulating free-field conditions. The aeroacoustic test section has been previously characterized and used for other acoustic experiments [81,82]. The chambers have lateral doors used to access the inside and install mics or other data acquisition equipment. 


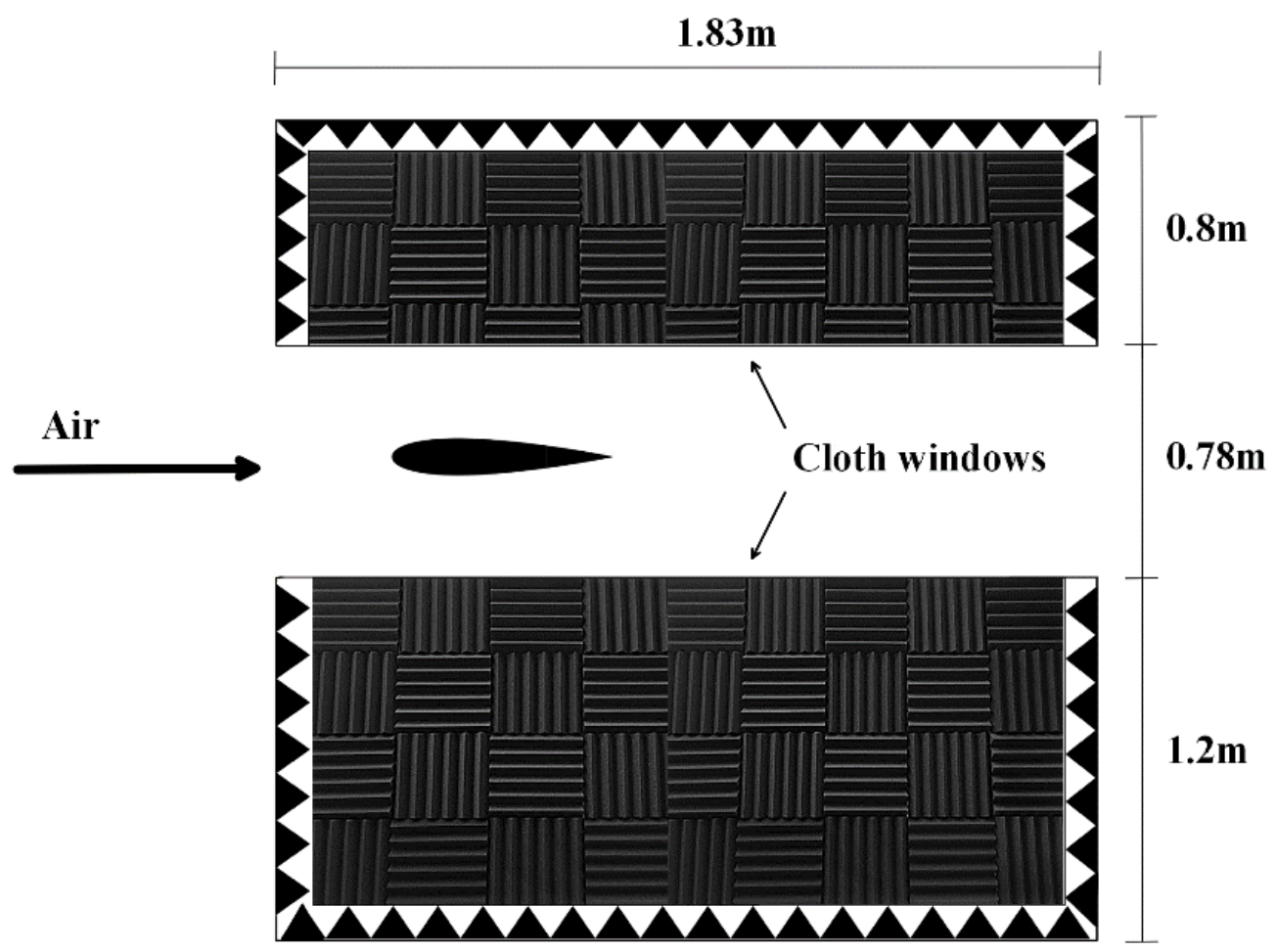

Figure 4: The aeroacoustic test section and anechoic chambers, as seen from above.

The upper and lower walls of the test section are composed of two aluminum sheet panels, and contain flushed, circular aluminium panels for the vertical mounting of a two-dimensional airfoil. The circular panels can be rotated in the clockwise or counter-clockwise directions to simulate the effect of having different AOAs. The tested airfoils are placed in the middle of the test section, and $0.45 \mathrm{~m}$ from the upstream end of the test section.

\subsection{Airfoil Design}

The airfoils under experimental investigation are NACA0012 airfoils equipped with different TE designs. The chord length of the airfoil is $300 \mathrm{~mm}$, and the span is $510 \mathrm{~mm}$. Between the LE, $x / c=0$, and $x / c=0.73$, the original NACA0012 airfoil profile is unmodified, where $\mathrm{x}$ is the streamwise direction. Further downstream, $0.73 \leq x / c \leq 1.0$, is a section that can be removed and 
replaced by either an unmodified or modified TE profiles. The lateral sides of the airfoil have holes that can be used to fix the airfoil to the circular rotating aluminium panel previously described. The airfoil was covered with sand-paper strip of thickness $5 \mathrm{~cm}(x / c=1.3 \%$ to $x / c=3 \%)$ to trip the BL and ensure developed turbulence at the airfoil TE.

Following the numerical analysis of TE finlets detailed in Chapter 5, and since finned airfoils haven't been tested in the Carleton University medium speed wind tunnel, TE finlets were chosen for experimental investigation. The TE of interest was machined out of a $3.2 \mathrm{~cm}$ by $11.4 \mathrm{~cm}$ aluminium square bar of length $2 \mathrm{ft}$ at the Carleton University machine shop using a 3 -axis computerized numerical control $(\mathrm{CNC})$ milling machine. The designed finlets are symmetric on both sides of the airfoil, extending from $x / c=0.73$ to $x / c=1$. They have a maximum height, $\mathrm{h}=10 \mathrm{~mm}$, measured from the $\mathrm{x}$-axis, at $x / c=0.96$. The addition of finlets does not increase the frontal projected area of the airfoil, thus minimizing the effect of finlets on pressure-drag. The thickness of the finlets is $1 \mathrm{~mm}$ and the spacing between each two consecutive finlets is $3.83 \mathrm{~mm}$, as seen in the drawing sheet shown in Figure 5. The manufactured finned TE is shown in Figure 6. Once attached to the main body, the finned TE section forms a continuous profile giving the appearance that the finlets are added directly to the main body of the NACA0012 airfoil, as seen in Figure 7. 

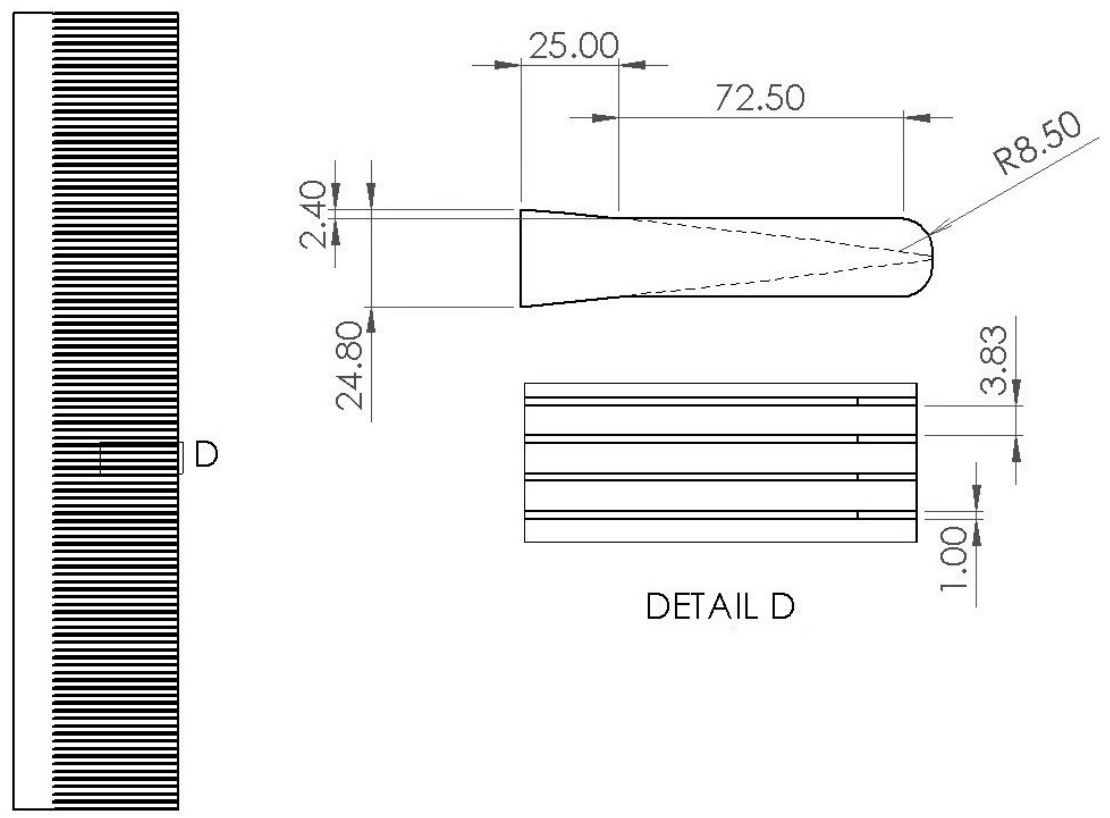

Figure 5: Drawing sheet showing details of the manufactured finned TE, units in $\mathrm{mm}$, not to scale.
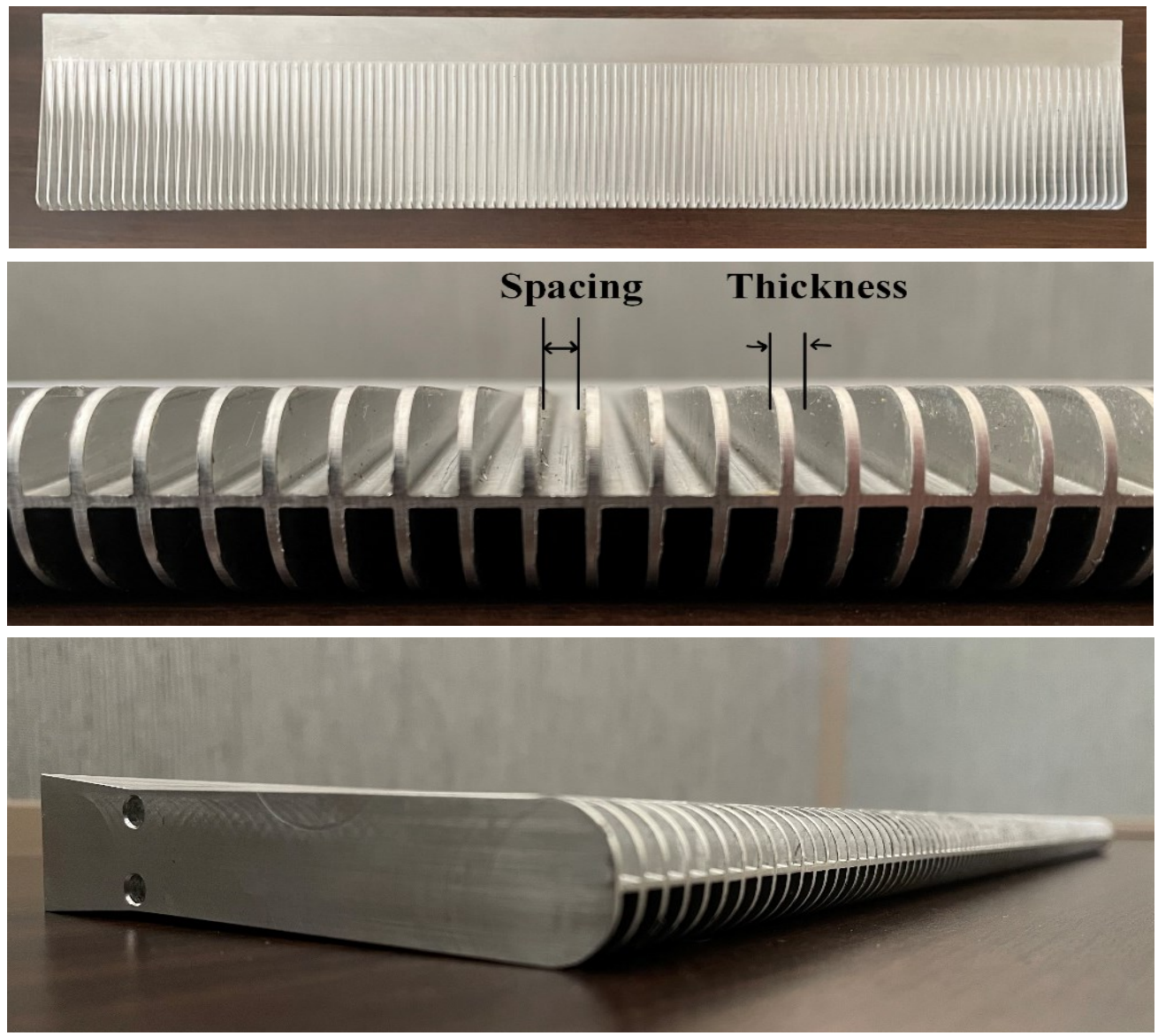

Figure 6: Manufactured finned NACA0012 airfoil TE. 


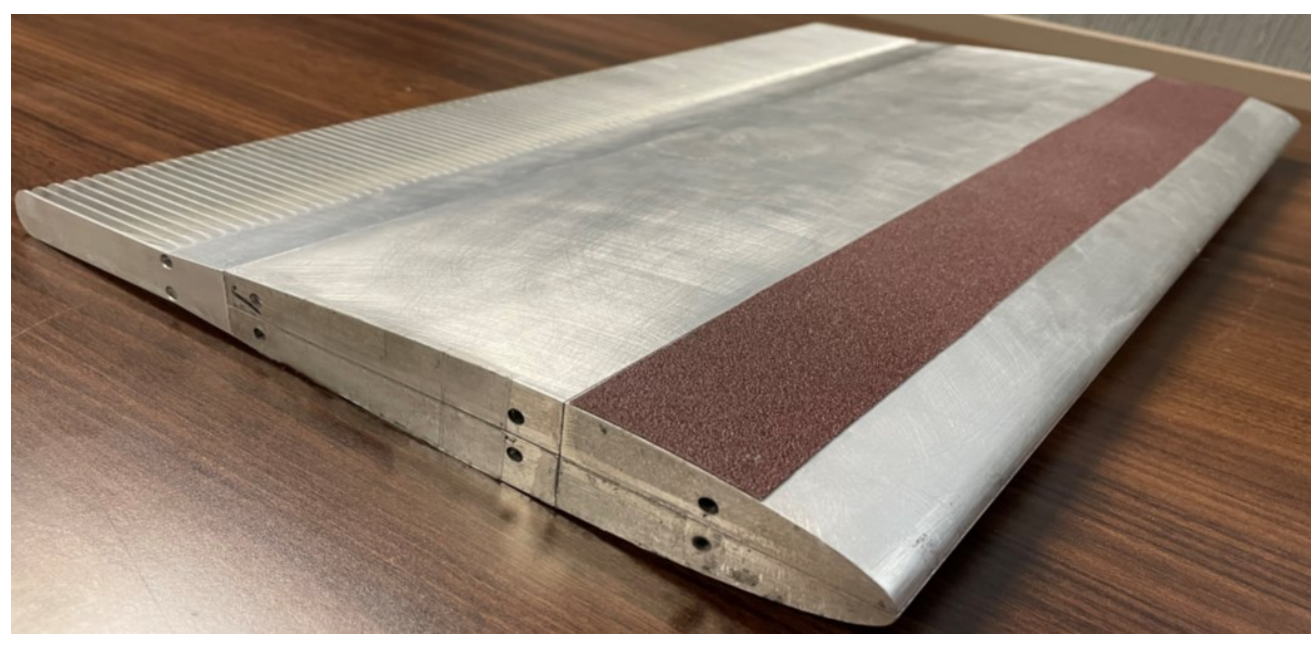

Figure 7: Fully assembled NACA0012 airfoil with TE finlets.

\subsection{Wind Tunnel Setup and Experimental Techniques}

The designed airfoil is vertically mounted in the test section, $0.45 \mathrm{~m}$ downstream of the test section entrance, between two rotating aluminium sidepanels. The airfoil AOA can be modified by rotating the circular side-panels around their axis. For the current study, the airfoil was tested at AOAs of $-10^{\circ},-5^{\circ}, 0^{\circ}, 5^{\circ}$ and $10^{\circ}$. The airfoil of interest is a NACA0012 airfoil modified with TE finlets. The inlet free jet velocity, $u=24 \mathrm{~m} / \mathrm{s}$, and the turbulence intensity is around $0.27 \%$. The corresponding chord-based Reynold's number $\left(\operatorname{Re}_{c}\right)$ is approximately 500,000. The BL is tripped using a sand-paper strip covering a portion of the airfoil LE, as discussed in the previous subsection. Tripping the BL ensures flow transition to turbulence before reaching the TE, and is similar in effect to the VM used to inject artificial turbulence in the numerical simulations. Figure 8 shows the airfoil at $0^{\circ}$ $\mathrm{AOA}$, as seen from the top of the test section with the upper rotating aluminium panel removed. 


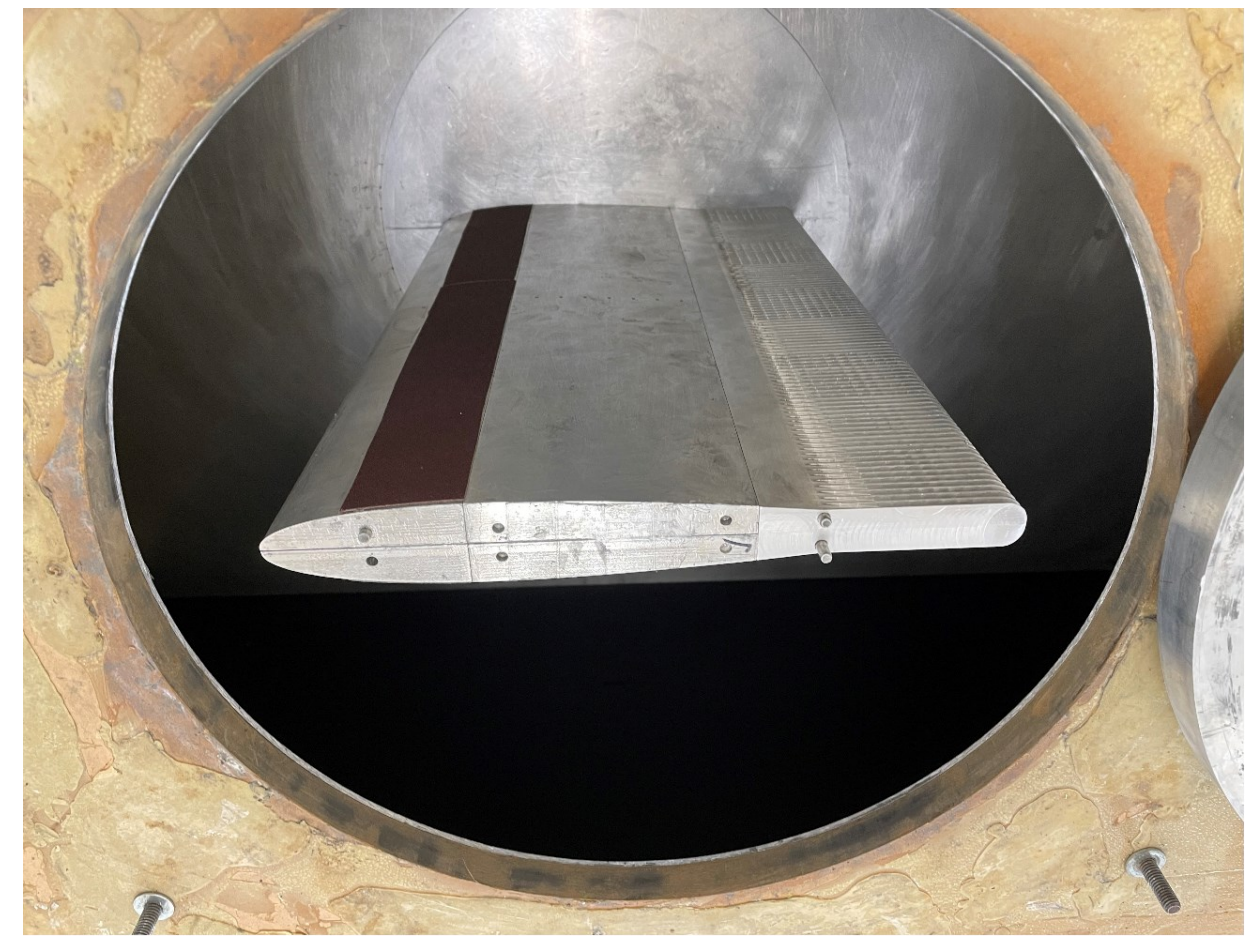

Figure 8: NACA0012 airfoil with the finned TE attached, as seen with the upper aluminum panel removed. Flow direction is from left to right.

Far-field noise measurements are taken using a Brüel \& Kjær microphone. The microphone is placed mid-span, directly above the airfoil TE at a distance of $1.5 \mathrm{~m}$. Figure 9 demonstrates the AOA and microphone configurations. Signals from the microphone were amplified using a B\&K 122 Nexus amplifier, before digitally stored through an analogue to digital converter having a resolution of 24bits. 


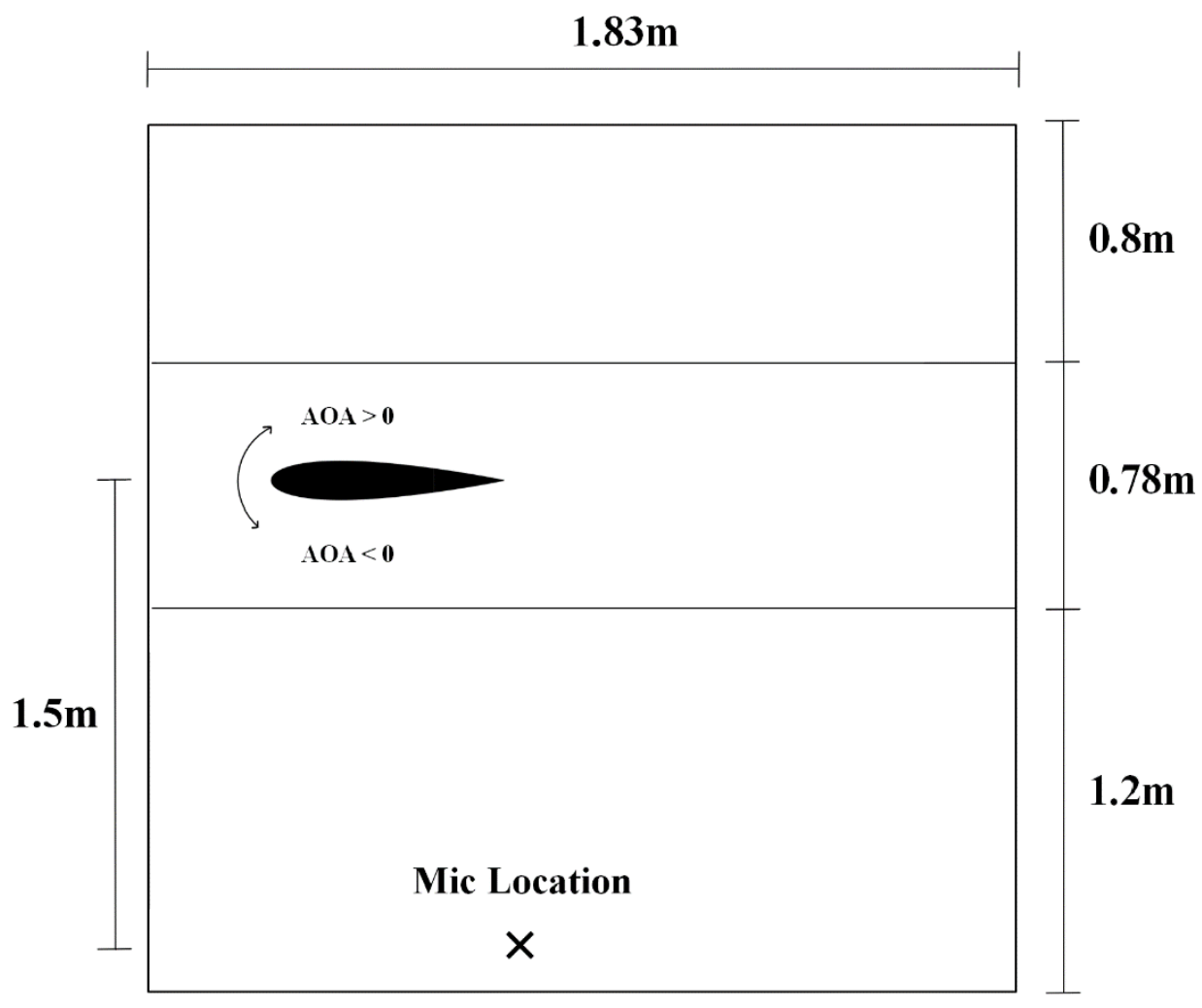

Figure 9: AOA configuration and microphone location.

The acoustic data sampling frequency, $f_{s}$, is set to $20 \mathrm{kHz}$ and the sampling period is 30 seconds. The corresponding frequency resolution, defined as the inverse of the sampling period, is equal to $0.033 \mathrm{~Hz}$. The acoustic data was sampled three times for each case to ensure consistency. The obtained signal is passed through a time-domain filter to remove the low and high frequency contamination, caused by the microphone's low frequency roll off and high-frequency aliasing. The band-pass filter used is a Butterworth filter with the first and second stopband frequencies of 200 and $f_{s} / 2 \mathrm{~Hz}$ respectively, where $f_{s}$ is the sampling frequency. The sound pressure level (SPL) is computed from the RMS of the filtered pressuretime signal, such that:

$$
S P L=10 \log _{10}\left(\frac{P_{R M S}^{2}}{P_{r e f}^{2}}\right)
$$

where $P_{\text {ref }}$ is approximately the lower limit of sensitivity of the human ear, $20 \mu \mathrm{Pa}$. 


\section{Chapter 4}

\section{Simulated TE Geometries and ELES Setup}

This chapter provides details about the simulated TE designs and their geometrical parameters. Details of the computational mesh are provided, as well as the simulation setup, domain segregation and boundary conditions. The purpose behind each simulation is outlined.

\subsection{TE Geometries}

The selected airfoil is a symmetric zero-lift NACA0012 airfoil, thanks to its popularity in literature and the availability of published results to compare the numerical predictions to. The base airfoil is supplemented with various noisesuppressing TE configurations.

Cases $\mathrm{C} 1.1$ and $\mathrm{C} 1.2$ are used to test two different ELES configurations. The first case, C1.1, where the letter " $\mathrm{C}$ " stands for computational, is a flat TE NACA0012 airfoil having a span of $18 \mathrm{~mm}$. The second case, C1.2, has a $30 \mathrm{~mm}$ span. For C1.1, the LES domain extends from $x / c=0.5$ to $x / c=1$, covering $50 \%$ of the airfoil chord while for C1.2, the LES domain extends from $x / c=0.7$ to $x / c=1$, covering $30 \%$ of the chord length. By reducing the streamwise length included in the LES zone in C1.2, a larger airfoil span can be simulated while keeping the number of elements under control. The origin is specified at the airfoil LE. Table 2 summarizes the geometric parameters of cases C1.1 and C1.2. The results are presented and compared in Chapter 5 , and the configuration used in C1.2 is selected for the proceeding simulations. 
Table 2: Characteristics of flat TE airfoils.

\begin{tabular}{cccc}
\hline Case & $\boldsymbol{c}(\boldsymbol{m m})$ & $\boldsymbol{s}(\boldsymbol{m m})$ & $\begin{array}{c}\text { \% of chord resolved using } \\
\text { LES }\end{array}$ \\
\hline $\mathbf{C 1 . 1}$ & 300 & 18 & $50 \%$ \\
\hline $\mathbf{C 1 . 2}$ & 300 & 30 & $30 \%$ \\
\hline
\end{tabular}

Standard serrations are commonly used to mimic the effect of the TE fringe found on barn owl wings. In order to study the effect of standard TE serrations and supplement experimental data previously acquired by B. Al Tlua [81,82], three different standard serration configurations are simulated. All of the serrations are cut directly into the TE of a realistic airfoil. This configuration offers better structural strength and integrity, but introduces partial bluntness, $\epsilon$, at the serration roots. A general model of standard serration characteristics is presented in Figure 10. Table 3 summarizes the geometric parameters of cases C2.1, C2.2 and C2.3. Among the three configurations, the serrations amplitude, $2 \mathrm{~h}$, and the serration wavelength, $\lambda$, are varied and their effect on the radiated tonal noise [25] is looked into. Changing $2 \mathrm{~h}$ translates into a change in $\epsilon$, and the same value of $2 \mathrm{~h}$ will give different values of $\epsilon$ for different types of airfoils. Furthermore, case C2.3 is meshed and simulated twice to demonstrate mesh convergence. 


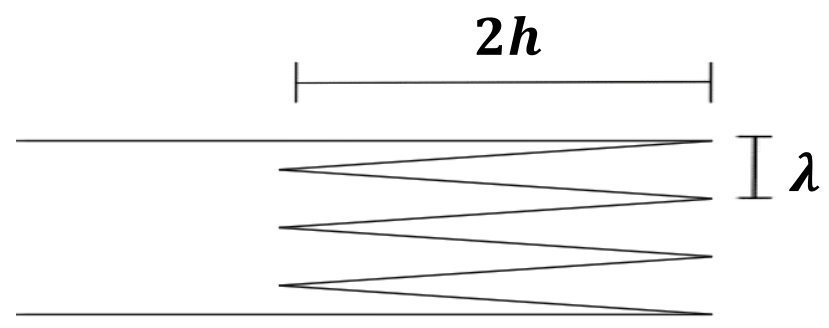

a) Top view

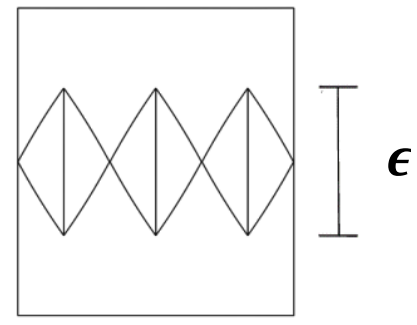

b) Back view

c) Isometric view

Figure 10: General model of standard TE serrations, not to scale.

Table 3: Geometric characteristics of standard TE serrations.

\begin{tabular}{cccccc}
\hline Case & $\boldsymbol{c}(\boldsymbol{m m})$ & $\boldsymbol{s}(\boldsymbol{m m})$ & $\mathbf{2 h}(\boldsymbol{m m})$ & $\boldsymbol{\lambda}(\boldsymbol{m m})$ & $\boldsymbol{\epsilon}(\boldsymbol{m m})$ \\
\hline $\mathbf{C 2 . 1}$ & 300 & 30 & 30 & 10 & 7.4 \\
\hline $\mathbf{C 2 . 2}$ & 300 & 30 & 60 & 7.5 & 16.3 \\
\hline $\mathbf{C 2 . 3}$ & 300 & 30 & 60 & 10 & 16.3 \\
\hline
\end{tabular}

Finlets are another noise-attenuating TE design that mimics the effect of the downy coat found on the surface of barn owl wings. Case C3 comprises a finned TE airfoil. To minimize the effect of the added finlets on the aerodynamics of the airfoil and avoid any substantial increase in pressure drag, the finlets are kept symmetric on both size of the airfoil, and are designed such that the frontal projected area does not increase. The thickness of the finlets, $t$, is $1 \mathrm{~mm}$ and the spacing between each two consecutive finlets, $S$, is $3.9 \mathrm{~mm}$. The finlets do not extend past the airfoil TE, and their LE falls at $x / c=0.73$. The maximum height of the finlets $(H)$, as measured from the airfoil surface, is $8 \mathrm{~mm}$, and is reached at $x / c=0.975 .(H)$. The origin is defined at the airfoil LE. The design parameters are 
inspired by the work of Bodling et al. [34] and Shi et al. [31]. Figure 11 shows a general model of TE finlets.

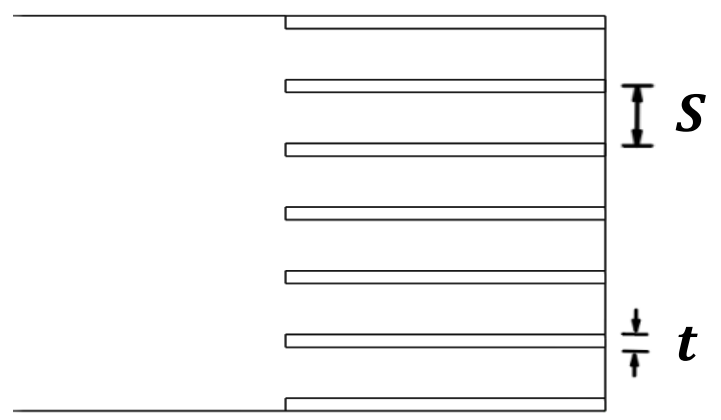

a) Top view

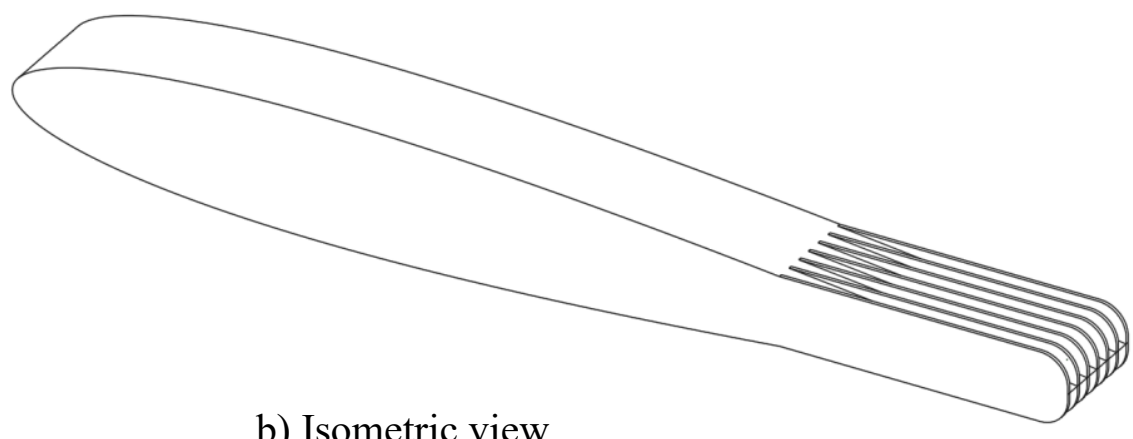

Figure 11 : General model of TE finlets, not to scale.

To achieve silent flight, owl wings utilize different noise-reducing TE concepts simultaneously. This poses an excellent example of how the different noise-reducing mechanisms observed by scientists can co-exist, and potentially supplement each other. Following the successful simulations of finlets and serrations, the concept of superimposing their noise-attenuating mechanisms is explored. By introducing finned serrations, case $\mathrm{C} 4$ is an attempt to mimic the combined effects of low-noise owl wings. First, serrations are cut directly into the airfoil TE, then finlets are added. The serration amplitude, $2 \mathrm{~h}=60 \mathrm{~mm}$ and the wave length, $\lambda=10 \mathrm{~mm}$. The serration root bluntness, $\epsilon$, is $16.3 \mathrm{~mm}$. The introduced finlets have a spacing, $S=5 \mathrm{~mm}$ and a thickness $t=1 \mathrm{~mm}$. A general model of the finned serration characteristics is presented in Figure 12. 


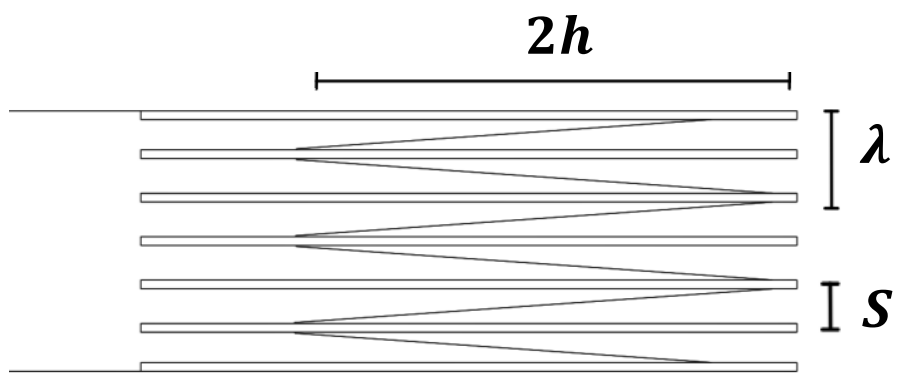

a) Top view

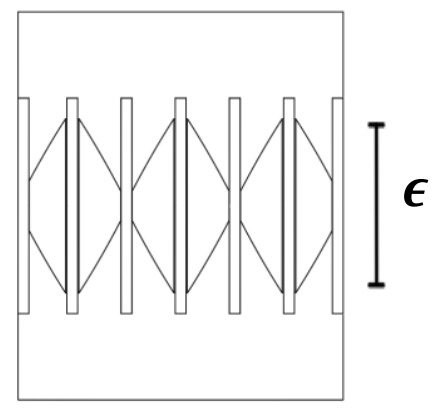

b) Back view

c) Isometric view

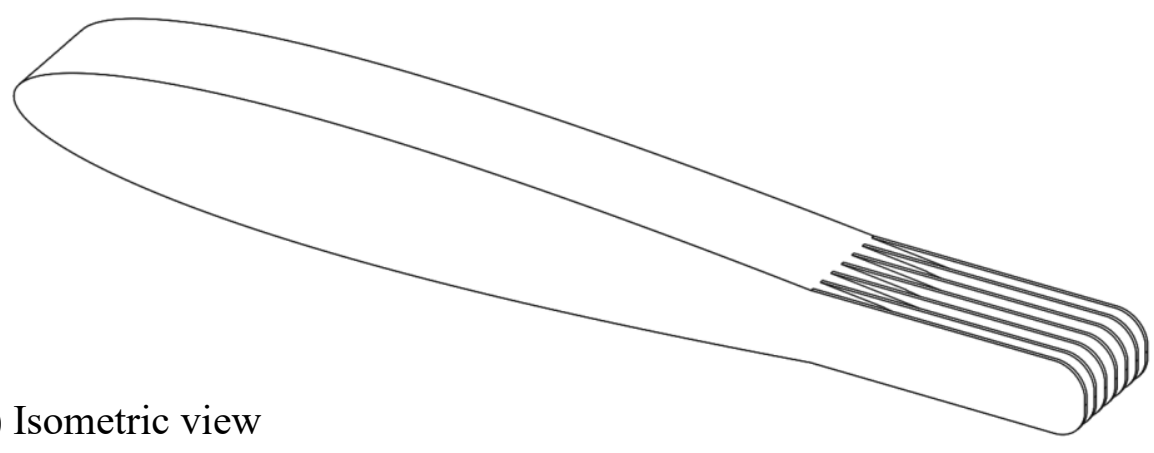

Figure 12: General model of finned TE serrations, not to scale.

It was observed in many promising noise-reducing TE designs that the most significant drawback is a narrowband tonal peak that appears. To address this issue, case C5 looks into the suppression of that tonal peak. Slanted-root serrations, initially proposed by Chen et al. [28], are simulated and their effects on the airfoil wake investigated. The results are compared to those of standard serrations. In the initial design phase, slanted-root serrations (C5) are very similar to the standard serrations found in C2.3. They have a serration amplitude, $2 h=60 \mathrm{~mm}$ and a serration wavelength, $\lambda=10 \mathrm{~mm}$. The unique parameter of slanted-root serrations is the slanting ratio, $h_{2} / h_{1}$. Figure 13 demonstrates the geometrical characteristics of slanted-root serrations, in which $h_{1}$ and $h_{2}$ take turns with the values $30 \mathrm{~mm}$ and $60 \mathrm{~mm}$ every wavelength, $\lambda$. Thus, the value of the slanting ratio, $h_{2} / h_{1}$, alternates between 0.5 and 2 . 


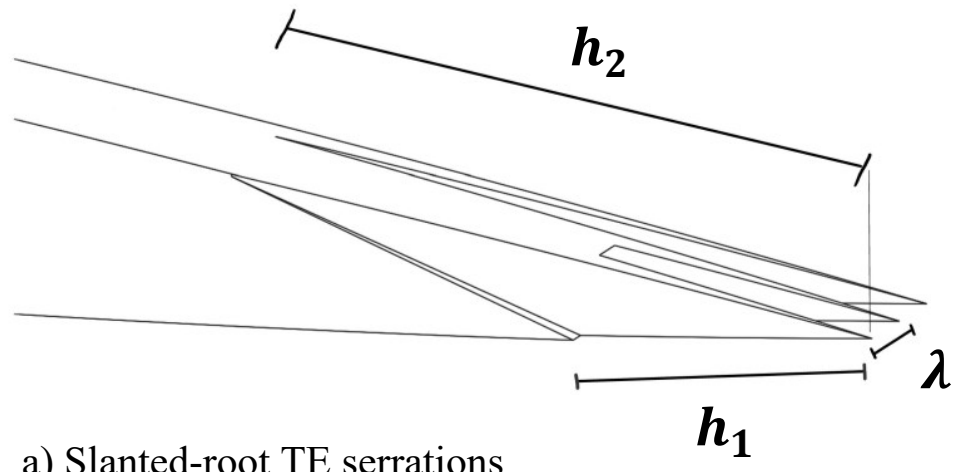

a) Slanted-root TE serrations

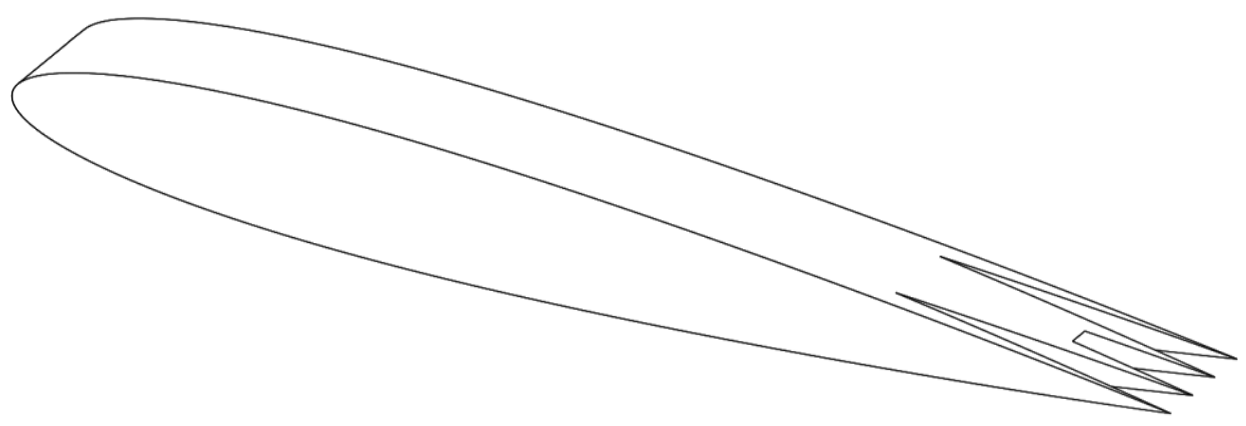

b) Isometric view

Figure 13: General model of slanted-root TE serrations, not to scale.

Bio-inspired TE designs are simulated for different purposes. Table 4 summarizes all the simulated cases, their most prominent geometric features, and the collective objective of each group of simulations. The following subsection explains the used meshing technique and the corresponding mesh parameters, then subsection 3.3 details the flow configuration and the setup of ELES in detail.

Table 4: Summary of all presented simulations.

\begin{tabular}{|c|c|c|c|}
\hline Case & $\begin{array}{c}\text { TE } \\
\text { Design } \\
\end{array}$ & $\begin{array}{c}\text { Main } \\
\text { Characteristics }\end{array}$ & Objective(s) \\
\hline \multirow{2}{*}{$\begin{array}{l}\text { C1.1 } \\
\text { C1.2 }\end{array}$} & \multirow{2}{*}{ Flat TE } & $\begin{array}{c}c=300 \mathrm{~mm} \\
s=18 \mathrm{~mm}\end{array}$ & \multirow{2}{*}{$\begin{array}{l}\text { - Develop the ELES } \\
\text { framework. } \\
\text { - Compare different ELES } \\
\text { configurations and determine } \\
\text { the best one. } \\
\text { - Assess the applicability of } \\
\text { ELES for the proposed }\end{array}$} \\
\hline & & $\begin{array}{c}c=300 \mathrm{~mm} \\
s=30 \mathrm{~mm}\end{array}$ & \\
\hline
\end{tabular}




\begin{tabular}{|c|c|c|c|}
\hline & & & $\begin{array}{l}\text { problem and compare with } \\
\text { experimental data. }\end{array}$ \\
\hline \multirow{3}{*}{$\begin{array}{l}\mathrm{C} 2.1 \\
\mathrm{C} 2.2 \\
\mathrm{C} 2.3\end{array}$} & \multirow{3}{*}{$\begin{array}{l}\text { Standard } \\
\text { TE } \\
\text { serrations }\end{array}$} & $\begin{array}{c}2 \mathrm{~h}=30 \mathrm{~mm} \\
\lambda=10 \mathrm{~mm} \\
\epsilon=7.4 \mathrm{~mm}\end{array}$ & \multirow{3}{*}{$\begin{array}{l}\text { - Assess the ability of ELES to } \\
\text { predict tonal noise } \\
\text { components. } \\
\text { - Supplement experimental data } \\
\text { previously acquired by B. Al } \\
\text { Tlua [81,82]. } \\
\text { - Investigate the effects of } \lambda \text {, } \\
2 h \text { and } \epsilon \text { on flow features and } \\
\text { narrowband peaks. } \\
\text { C2.3 is meshed and simulated } \\
\text { twice to demonstrate mesh } \\
\text { convergence. }\end{array}$} \\
\hline & & $\begin{array}{c}2 h=60 \mathrm{~mm} \\
\lambda=7.5 \mathrm{~mm} \\
\epsilon=16.3 \mathrm{~mm}\end{array}$ & \\
\hline & & $\begin{array}{c}2 h=60 \mathrm{~mm} \\
\lambda=10 \mathrm{~mm} \\
\epsilon=16.3 \mathrm{~mm}\end{array}$ & \\
\hline C3 & TE finlets & $\begin{array}{c}S=3.9 \mathrm{~mm} \\
t=1 \mathrm{~mm}\end{array}$ & $\begin{array}{l}\text { Identify the noise reducing } \\
\text { mechanisms of finlets on a } \\
\text { fundamental level and } \\
\text { compare them to those } \\
\text { observed in standard } \\
\text { serrations cases. } \\
\text { Use the results as to determine } \\
\text { whether to experimentally test } \\
\text { TE finlets and investigate } \\
\text { their performance at different } \\
\text { AOAs. }\end{array}$ \\
\hline C4 & $\begin{array}{c}\text { Finned } \\
\text { TE } \\
\text { serrations }\end{array}$ & $\begin{array}{c}2 h=60 \mathrm{~mm} \\
\lambda=10 \mathrm{~mm} \\
\epsilon=16.3 \mathrm{~mm} \\
S=5 \mathrm{~mm} \\
t=1 \mathrm{~mm}\end{array}$ & $\begin{array}{l}\text { Investigate the possibility of } \\
\text { superimposing the different } \\
\text { noise-attenuating flow } \\
\text { mechanisms observed in } \\
\text { standard serrations and finlets, } \\
\text { as seen with owl wings. }\end{array}$ \\
\hline C5 & $\begin{array}{l}\text { Slanted- } \\
\text { root TE } \\
\text { serrations }\end{array}$ & $\begin{array}{c}2 h=60 \mathrm{~mm} \\
\lambda=10 \mathrm{~mm} \\
h_{1}=30 \sim 60 \mathrm{~mm} \\
h_{2}=60 \sim 30 \mathrm{~mm} \\
h_{2} / h_{1}=0.5 \sim 2\end{array}$ & $\begin{array}{l}\text { - Attempt to eliminate or reduce } \\
\text { the tonal noise observed in } \\
\text { standard serrations. } \\
\text { - Understand and visualize the } \\
\text { effect of slanted-roots on } \\
\text { vortex shedding. }\end{array}$ \\
\hline
\end{tabular}




\subsection{Computational Mesh}

In the initial phases of this work, several meshing techniques were considered, and after some trial and error, the cartesian cut-cell method was found most appropriate for the current studies. The cut-cell method has received significant development in recent years [83], and results in predominantly hexahedral meshes. The generated hexahedral elements yield a smaller number of elements for the same mesh resolution, when compared to other mesh types, thus significantly reducing the required computational power. In addition, the resulting elements are characterized by their high orthogonal quality and low skewness, which minimizes truncation errors $[83,84]$.

One of the key ideas of the ELES method is to predefine different zones with different treatments of turbulence in the pre-processing stage of the simulation. ELES an infrastructure that combines two existing methods in a zonal approach. The flow domain is divided to RANS and LES zones. Since RANS and LES are fundamentally different models, as explained in Chapter 2, the mesh recommendations for each zone are the same as those applicable to the individual models [85]. The part of the domain where the turbulence is modeled using RANS equations is covered by a suitable RANS grid. For acoustic simulations, it is important to resolve the small-scale turbulent structure in the vicinity of the airfoil surface, which poses stricter mesh requirements on the LES zone when compared to RANS. The conversion from modeled turbulence to resolved turbulence is achieved by the introduction of synthetic turbulence at the RANS/LES interface. For that purpose, the VM [79] is chosen. Element size is restricted to $25.6 \mathrm{~mm}$ in the coarse RANS zone, $0.8 \mathrm{~mm}$ in the refined LES zone and $0.2 \mathrm{~mm}$ on the airfoil surface in the vicinity of the TE. Elements in the airfoil wake of the RANS zone have a size of $5 \mathrm{~mm}$. 40 inflation layers are generated around the airfoil with the thickness of the first layer set to $7.6 * 10^{-3} \mathrm{~mm}$ and a growth factor of 1.08 , thus ensuring $y^{+}<0.5$ everywhere on the airfoil surface, at least 3 layers in the viscous sublayer and overall accurate boundary layer resolution. The grid resolution in 
terms of wall-normal units is defined by $\Delta x^{+}=\frac{u_{t} \Delta x}{v}, \Delta y^{+}=\frac{u_{t} \Delta y}{v}$ and $\Delta z^{+}=$ $\frac{u_{t} \Delta z}{v}$, where $u_{\tau}$ is the frictional velocity and $v$ is the kinematic viscosity. The generated computational grids have a maximum resolution $\Delta x_{\max }^{+} \leq 20$ and $\Delta z_{\text {max }}^{+} \leq 20$ in the streamwise and spanwise directions, respectively $[85,86]$. A steady-state mesh convergence study was carried out by progressively refining the mesh and comparing the values of integrated output parameters (such as lift and drag coefficients). Three different meshes, A, B and C were generated as shown in Table 5. Figure 14 shows the element distribution of mesh A, and Figure 15 gives the instantaneous $y^{+}$values for the same mesh. The resulting meshes resolve $80 \%$ or more of the flow turbulent kinetic energy. The maximum error between integrated values, such as lift and drag coefficients, was less than $0.4 \%$, and the overall effect of the mesh on the results was found to be negligible.

Table 5: Characteristics of meshes used in mesh convergence study.

\begin{tabular}{cc}
\hline Mesh & Number of Elements \\
\hline $\mathrm{A}$ & $6,666,668$ \\
\hline $\mathrm{B}$ & $7,606,083$ \\
\hline $\mathrm{C}$ & $9,011,531$ \\
\hline
\end{tabular}

In addition, meshes $\mathrm{A}$ and $\mathrm{B}$ were carried over for a transient simulation analysis. The lift-history coefficients were evaluated for each mesh at every time step and their RMS values were computed. Both meshes yield the same liftcoefficient RMS value, $c_{L_{R M S}}=0.0013$, with a maximum error of less than $1 \%$. Since ELES is a two-step process, where a steady-state RANS simulation feeds information to a high-resolution transient LES simulation, it is important to ensure mesh convergence in both scenarios. Obtaining consistent results in terms of integrated flow parameters, for both steady-state and transient simulations, is a strong indication of the convergence of the used computational mesh, i.e. the mesh directly resolves enough flow structures for the results not to change with mesh refinement. In Chapter 5, results are compared against experimental data, and mesh convergence is further demonstrated as case C2.3 is simulated twice and quantities of interest, such as lift-coefficient history and tonal peak occurrence and frequency, 
are looked into and compared. Table 6 lists the number of elements corresponding to each of the presented simulations.

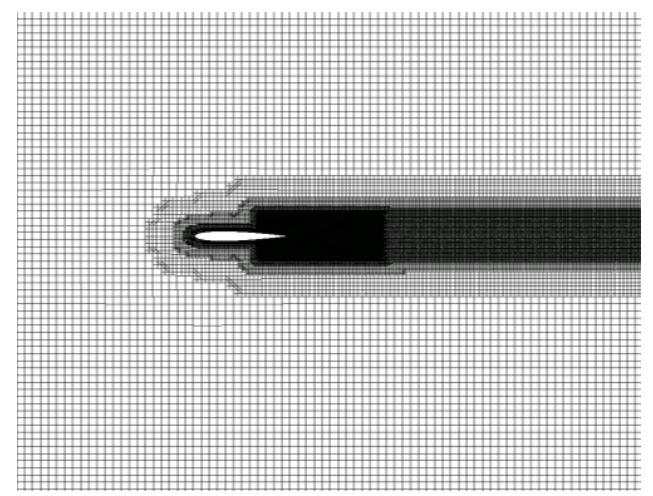

a) Mesh

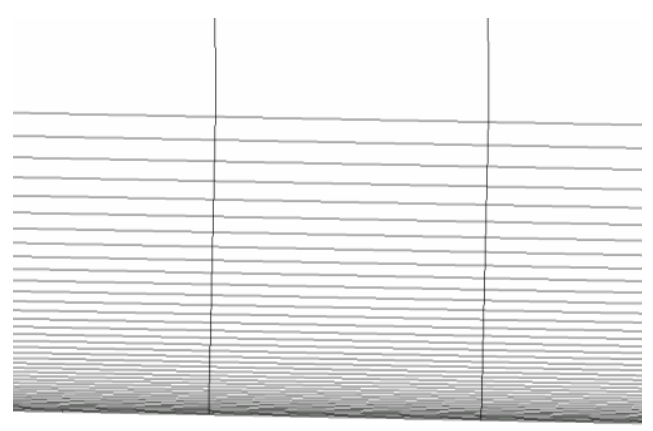

b) Inflation layers around airfoil

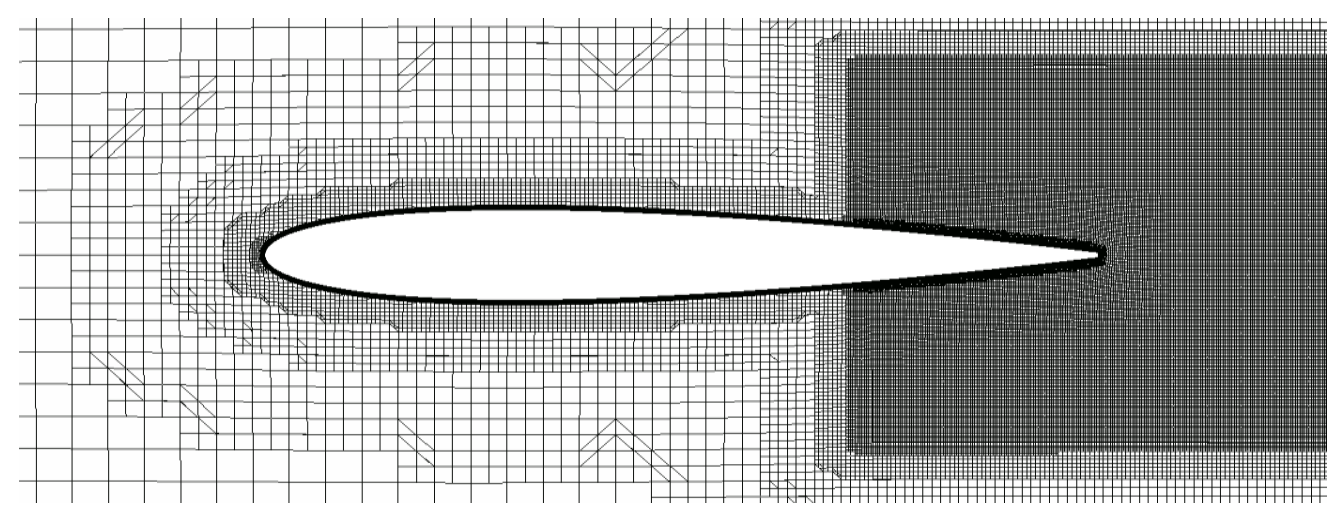

c) Mesh near airfoil surface

Figure 14: Computational mesh.
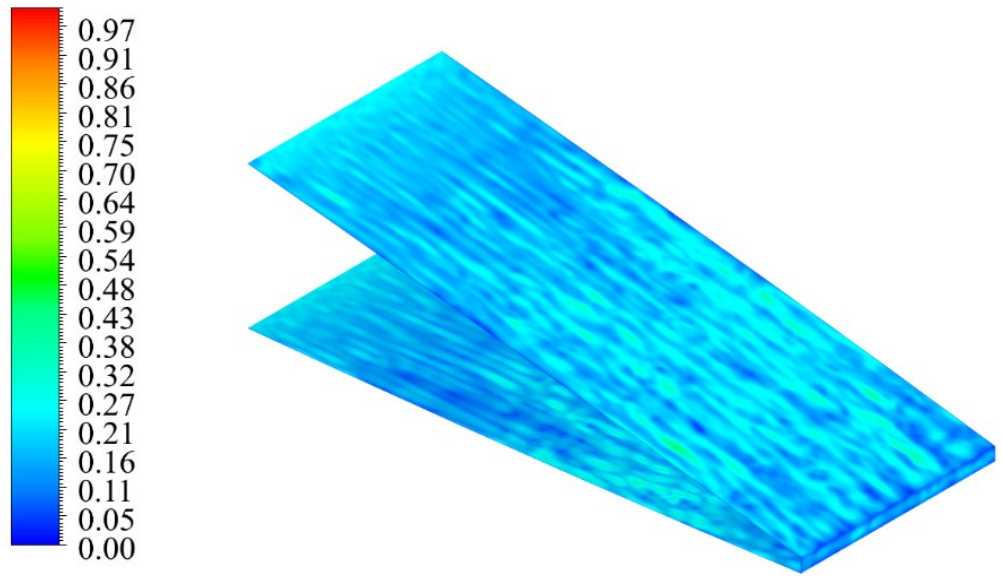

Figure 15: Instantaneous $y^{+}$distribution. 
Table 6: Number of elements in each computational mesh.

\begin{tabular}{cccc}
\hline Case & RANS & LES & Total \\
\hline $\mathrm{C} 1.1$ & 456,353 & $4,586,780$ & $5,043,133$ \\
\hline $\mathrm{C} 1.2$ & 976,300 & $5,690,368$ & $6,666,668$ \\
\hline $\mathrm{C} 2.1$ & 976,300 & $6,134,898$ & $7,111,198$ \\
\hline $\mathrm{C} 2.2$ & 976,300 & $5,896,422$ & $6,872,722$ \\
\hline $\mathrm{C} 2.3$, Mesh 1 & 976,300 & $5,647,833$ & $6,624,133$ \\
\hline $\mathrm{C} 2.3$, Mesh 2 & $1,050,433$ & $6,173,725$ & $7,224,158$ \\
\hline $\mathrm{C} 3$ & 976,300 & $7,772,301$ & $8,748,601$ \\
\hline $\mathrm{C} 4$ & 976,300 & $7,936,811$ & $8,913,111$ \\
\hline $\mathrm{C} 5$ & 976,300 & $6,462,529$ & $7,438,829$ \\
\hline
\end{tabular}

\subsection{ELES Setup and Flow Configuration}

To ensure the accuracy of CFD simulations, it is important to specify a simulation domain large enough to mimic free-stream air flow conditions. If the flow field is too small, the prescribed boundary conditions can adversely affect the simulation results [87]. At the same time, the span of the simulated domains must be kept smaller than that of experimental testing to avoid excessive computational power requirements. All the performed simulations are three-dimensional in nature.

The simulated airfoils are placed in a square $10 c \times 10 c$ domain. The flow domain is divided into two regions as seen in Figure 16. RANS equations are employed in a coarse RANS domain, while LES equations are employed in a refined LES region near the TE. Two embedded domain configurations are initially tested through $\mathrm{C} 1.1$ and $\mathrm{C} 1.2$. For cases C1.1 and C1.2, the LES domains in the streamwise direction extend from $x / c=0.5$ and $x / c=0.7$, respectively, to $1 c$ downstream of the TE. The origin is defined at the airfoil LE. In the transverse direction, the LES domain extends $0.25 c$ above and below the airfoil. Computations are carried out at $0 \mathrm{AOA}$, a free stream velocity $u_{\infty}=24 \mathrm{~m} / \mathrm{s}$ and a free stream Mach number $M_{\infty}=0.071$, resulting in a chord-based Reynolds number, $R e_{c}=$ $\frac{\rho u_{\infty} D}{\mu}$, of approximately 500,000 , where $\rho$ is the fluid density, $\mu$ is the dynamic 
viscosity and $D$ is the characteristic length, which is the airfoil chord " $c$ " in this case. To simulate higher AOAs, the LES domain size will have to be increased, which would require more computational power, and the RANS/LES interface would have to be strategically placed to avoid having it in regions of high gradients. Table 7 summarizes the flow parameters used in the simulations.

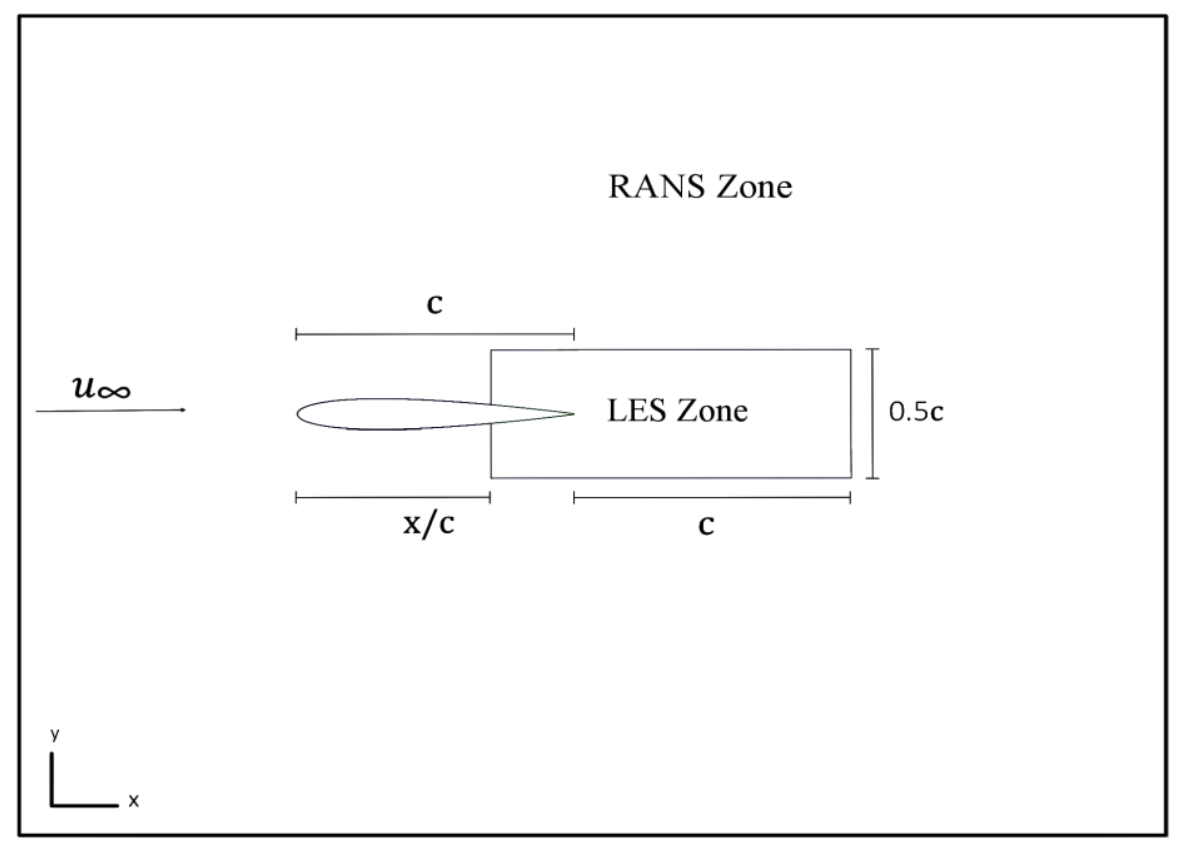

Figure 16: Schematic illustration of the segregated modeling domains.

Table 7: Flow Parameters.

\begin{tabular}{cc}
\hline$u_{\infty}$ & $24 \mathrm{~m} \mathrm{~s}^{-1}$ \\
\hline$M_{\infty}$ & 0.071 \\
\hline$R e_{c}$ & 500,000 \\
\hline$\mu$ & $1.7894 * 10^{-5} \mathrm{~kg} \mathrm{~m}^{-1} \mathrm{~s}^{-1}$ \\
\hline$\rho$ & $1.225 \mathrm{~kg} \mathrm{~m}^{-3}$ \\
\hline$A O A$ & 0 \\
\hline
\end{tabular}

The boundary conditions used are shown in Figure 17. A velocity inlet boundary condition is specified at the domain entrance, where $u_{\infty}=24 \mathrm{~m} / \mathrm{s}$. The selected velocity ensures that the obtained Re is around 500,000 and turbulent, and matches the speed limitations for acoustic testing at the Carleton University medium speed wind tunnel. Periodic boundary conditions (PBCs) are applied on 
the right and left side walls of the domain in the spanwise direction to allow the flow to develop naturally. No-slip boundary conditions are applied on the airfoil surface and a zero gauge-pressure outlet boundary condition is used. To mimic the effect of low-turbulence external air flow, the inlet turbulence intensity is set to $0.3 \%$. The SIMPLE pressure-velocity coupling scheme is used. This segregated algorithm is less computationally demanding that other coupled algorithms, and is recommended for iterative time advancement simulations, in which equations are solved iteratively for a given time-step until the convergence criteria are met. All results are second order accurate in time and space.

The flow is initialized using the k- $\omega$ SST model developed by Menter [73], as it gives accurate separation predictions for external flows. The VM [79] is then used to inject turbulence at the RANS/LES interface and the simulation is run for $4 T_{T F}$ "through-flow time" to obtain a fully developed flow, where $T_{T F}=\frac{L_{C F D}}{u_{\infty}}[85]$. $L_{C F D}$ is the LES domain length in the streamwise direction. WALE subgrid-scale (SGS) modelling is employed in the LES region as it is designed to return correct asymptotic wall behavior for wall-bounded flows [76]. The time step $d t=1.2 *$ $10^{-5}$ seconds. With these values, the Courant-Friedrichs-Lewy (CFL) number achieved is $\leq 1$ everywhere in the domain, meaning the flow particles don't travel

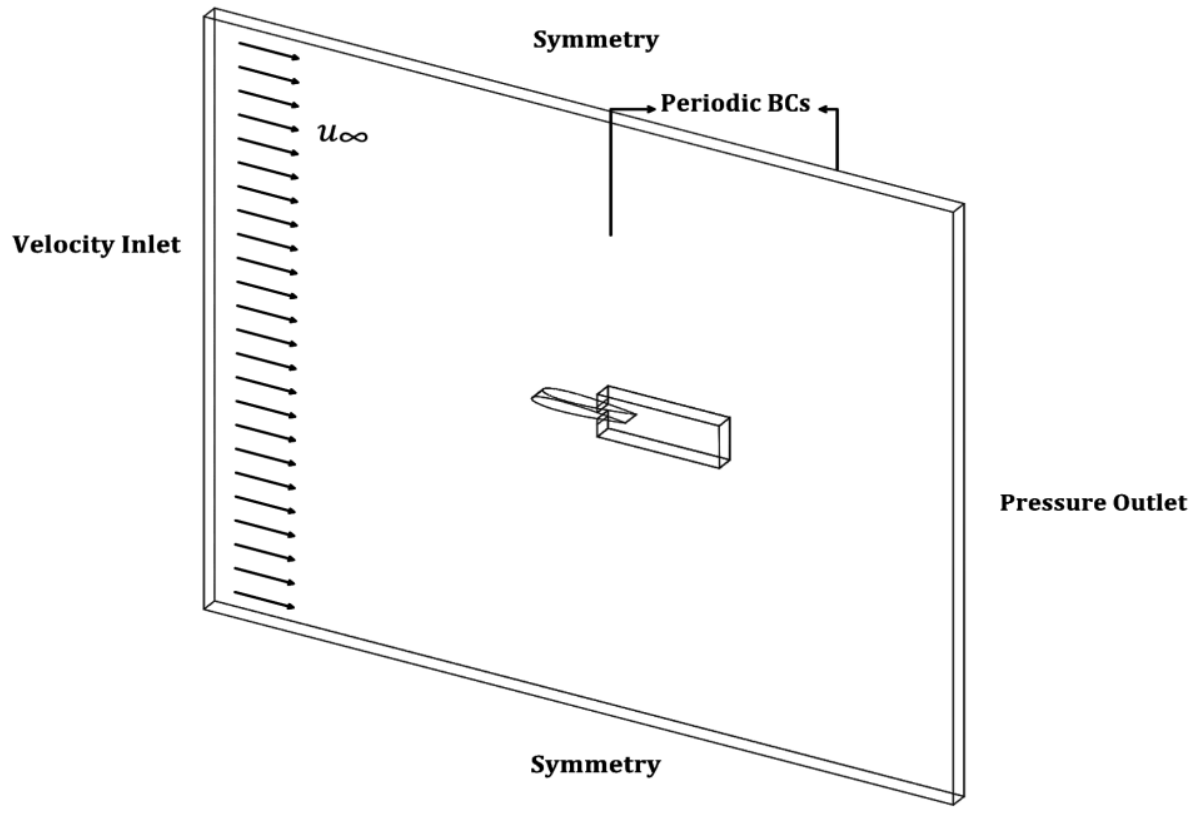

Figure 17: Boundary conditions. 
more than the length of one mesh element every time step. Residuals are reduced by three orders of magnitude each time step. Lastly, acoustic data is gathered for $3 T_{T F}$. All convergence residuals are set to $10^{-5}$. Pressure and velocity monitoring points are placed in the airfoil wake and statistical convergence is achieved. Statistical convergence is also achieved, in terms of the coefficients of lift and drag.

The FW-H aeroacoustic analogy [8] is used to compute the radiated far-field noise for all computational cases. Pressure fluctuations are propagated to receivers placed midspan at a distance of $1.5 \mathrm{~m}$ directly above the airfoils' TEs. Acoustic data is sampled every 2 flow-timesteps and data sampling is performed for $3 T_{T F}$ after the flow is fully developed, resulting in a sampling frequency of $41.67 \mathrm{kHz}$ and a frequency resolution of $28.4 \mathrm{~Hz}$, where the frequency resolution is defined as the inverse of the sampling period. Fast Fourier Transform (FFT) is performed on the resulting time signals to compute the SPL signal in the frequency domain. The Hanning window is applied to the time signals to reduce the numerical leakages associated with the discrete FFT [88]. All simulations are carried out using the commercial CFD software FLUENT 2019R3 and run on an Intel Xeon L5410 2.33 $\mathrm{GHz}$ platform of 60 cores at Carleton University.

In summary, ELES is adopted to reduce computational cost associated with high resolution aeroacoustic CFD simulations. The flow domain is split into 2 distinct zones: RANS and LES. The first only captures the average flow quantities, based on which artificial turbulence is introduced at the RANS/LES interface. The LES zone is responsible for the resolution of the small-scale turbulent structures responsible for sound generation. The flow simulation is divided into flow initialization, which lasts $4 T_{T F}$, and turbulence data sampling, which lasts $3 T_{T F}$. The time step is chosen to keep the CFL number below 1. The simulation results converge, and mesh convergence is tested and achieved. The FW-H aeroacoustic analogy is used to propagate the radiated noise to a receiver placed $1.5 \mathrm{~m}$ directly above the airfoil. The following chapter presents and discusses the numerical results and experimental data. 


\section{Chapter 5}

\section{Results and Discussion}

This chapter presents and discusses the numerical and experimental results. Subsection 5.1 shows the numerical predictions for the different TE designs simulated using ELES, for the purpose of analyzing and comparing the aerodynamic and aeroacoustic features of the different bio-inspired configurations. Surface pressure statistics are presented in 5.1.1. The turbulence kinetic energy (TKE) distributions at streamwise locations of interest in the vicinity of the airfoil surface and TE are shown in 5.1.2. The effect of each design on the wake characteristics and vorticity is shown in 5.1.3, followed by the far-field noise spectra in 5.1.4. Subsection 5.2 presents the results of the medium speed wind tunnel experiments, to analyse the effect of the AOA on noise reduction for TE finlets. Finally, a comparison between the numerical and experimental results is given subsection 5.3 .

\subsection{Numerical Results}

\subsubsection{Surface Pressure}

The pressure coefficient $\left(C_{p}\right)$ distribution around the airfoil is an important parameter in terms of boundary layer behavior because the pressure gradient affects the development of both laminar and turbulent boundary layers [89-91]. In addition, the TBL is responsible for the majority of the generated sound. $C_{p}$ distributions for cases $\mathrm{C} 1.1$ and $\mathrm{C} 1.2$ are computed and compared against experimental results obtained by Lee and Kang [92] for a NACA0012 airfoil at a $\operatorname{Re}=600,000$, and full 
LES results published by Marsden, Bogey and Bailly [93] at $\mathrm{Re}=500,000$, as seen in Figure 18. The LES domain in C1.1 is longer in the streamwise direction than $\mathrm{C} 1.2$, as it starts at a $x / c=0.5$. To keep the number of elements under control and simulate a larger span, the LES domain for C1.2 starts at $x / c=0.7$. The span of the simulated domains is kept smaller than that of experimental testing to avoid prohibitive computing power and simulation run-time. The airfoil span is $18 \mathrm{~mm}$ $(6 \% * c)$ in $\mathrm{C} 1.1$ and $30 \mathrm{~mm}(10 \% * c)$ in $\mathrm{C} 1.2$. Excellent agreement is achieved between the computational and experimental results; hence, both $\mathrm{C} 1.1$ and $\mathrm{C} 1.2$ are validated against existing literature. Although both configurations yield acceptable results, the configuration used in $\mathrm{C} 1.2$ is chosen as the base configuration and carried over for the proceeding simulations. Both configurations follow the general recommendation that RANS/LES interface should be more than 3 boundary layer thicknesses upstream from any strong non-equilibrium zones [85], but the spanwise domain of $\mathrm{C} 1.2$ covers more than 3 boundary layer thicknesses, which is important to avoid inaccuracies caused by the spanwise periodicity condition [85].

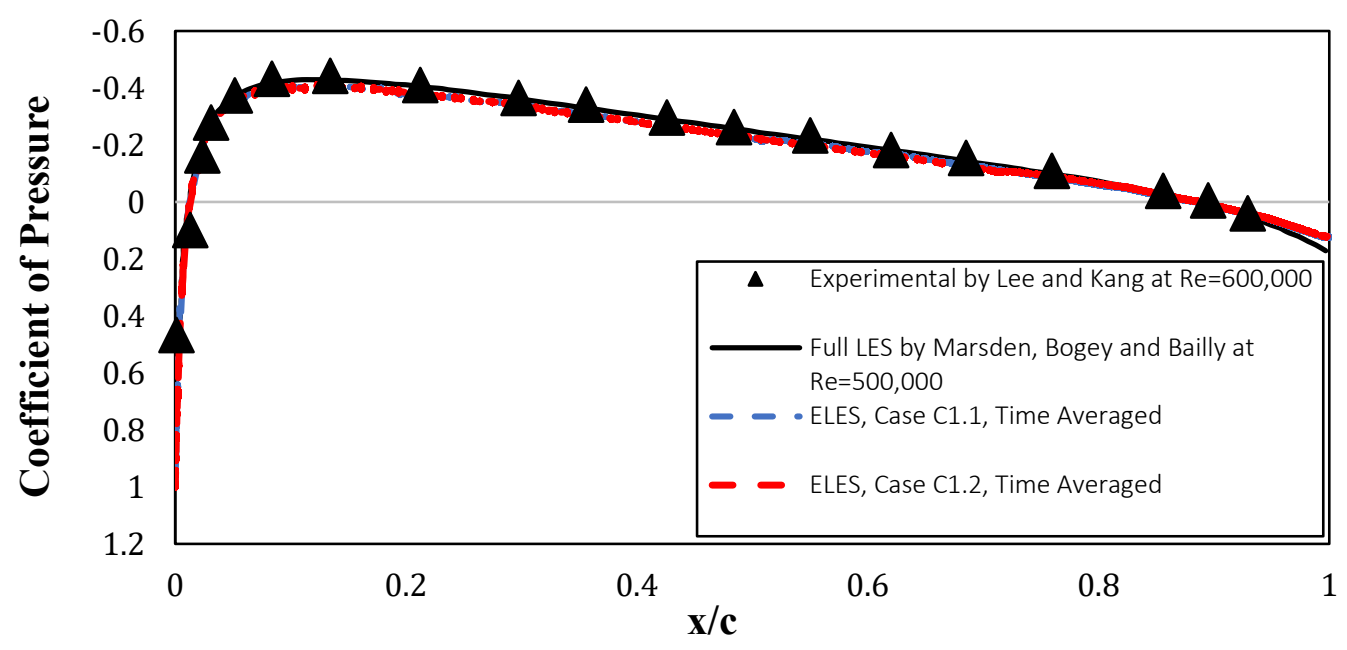

Figure 18: $C_{p}$ distribution on airfoil surface. 
Figure 19 shows the $P_{r m s}$ distribution on the airfoil surface for cases C1.2, $\mathrm{C} 2.1, \mathrm{C} 2.2, \mathrm{C} 2.3$ and $\mathrm{C} 5$. For case $\mathrm{C} 1.2, P_{r m s}$ increases in the streamwise direction as the boundary layer grows, and is maximum close to the sharp TE. In C2.1, C2.2 and $\mathrm{C} 2.3$, the maximum pressure fluctuation region changes and is concentrated downstream of the serration roots. The amplitude of the observed unsteady pressure fluctuations is highest in $\mathrm{C} 2.2$ and $\mathrm{C} 2.3$, where $\epsilon$ and $2 \mathrm{~h}$ are equal and greater than C2.1 $\left(\epsilon_{C 2.2, C 2.3}=16.3 \mathrm{~mm}, 2 h_{C 2.2, C 2.3}=60 \mathrm{~mm}, \epsilon_{C 2.1}=7.4 \mathrm{~mm}\right.$ and $2 h_{C 2.1}=30 \mathrm{~mm}$ ). This is where the pressure fluctuations are most violent, which suggests that aeroacoustic improvements to this design would require a modification of the flow field at that area. In other words, the majority of sound is generated near the serration roots, which agrees with the findings of [66], and further improvement to the design would be most effective if they address this phenomenon. C2.1 is subject to weaker $P_{r m s}$ than $\mathrm{C} 2.2$ and $\mathrm{C} 2.3$, but the values are still higher than C1.2 (flat TE). Results suggest that the maximum $P_{r m s}$ value increases as the serration amplitude, and subsequently the root bluntness, are increased. Figure 19f shows the $P_{r m s}$ distribution for the case of finlets $(\mathrm{C} 3)$, where maximum $P_{r m s}$ values occur near the extremity of the finlets themselves. This is explained by the time-averaged velocity contours shown in Figure 20, taken at a plane parallel to the $z-x$ plane. Two independent BLs are formed on the opposite faces of the finlets, and they collide for the first time at $x / c=1$. The abrupt interaction of the opposite BLs is responsible for the detected maximum $P_{r m s}$ values. Slanted-root serrations (C5) are subject to a smaller maximum $P_{r m s}$ value compared to standard serrations (C2.1, C2.2 and C2.3), and the region of maximum fluctuations is pushed further downstream, closer to where the slanted roots end. Weaker surface pressure fluctuations near the TE suggest the weakening of the shed vortices. 

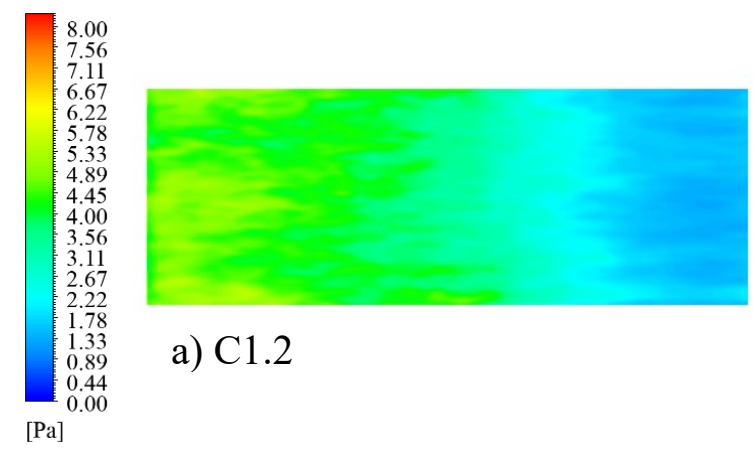

\section{a) $\mathrm{C} 1.2$}

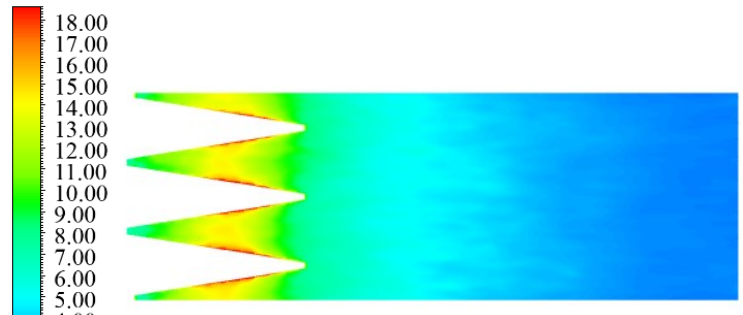

b) $\mathrm{C} 2.1$

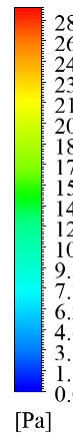

c) $\mathrm{C} 2.2$

[Pa]
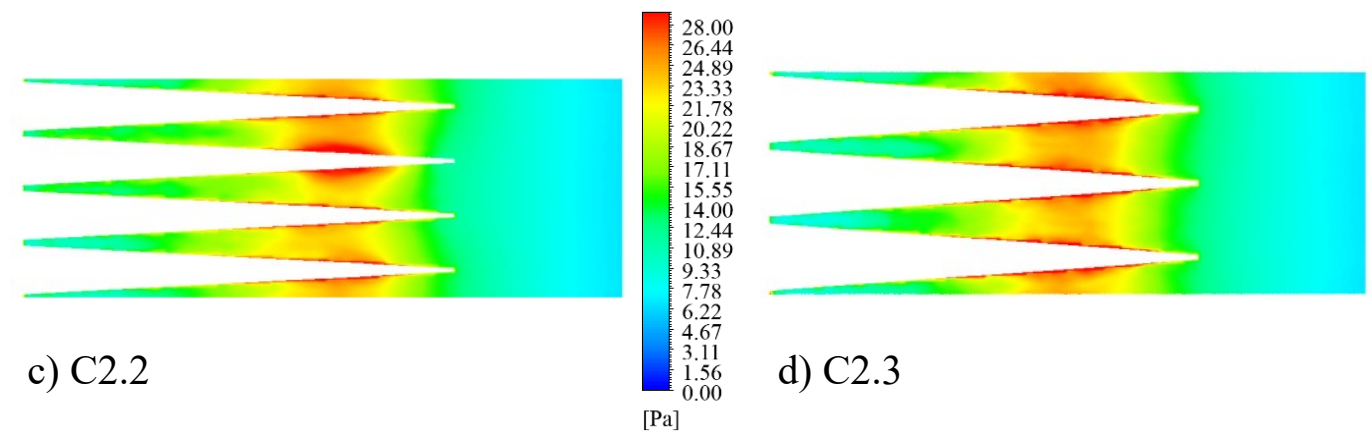

d) $\mathrm{C} 2.3$
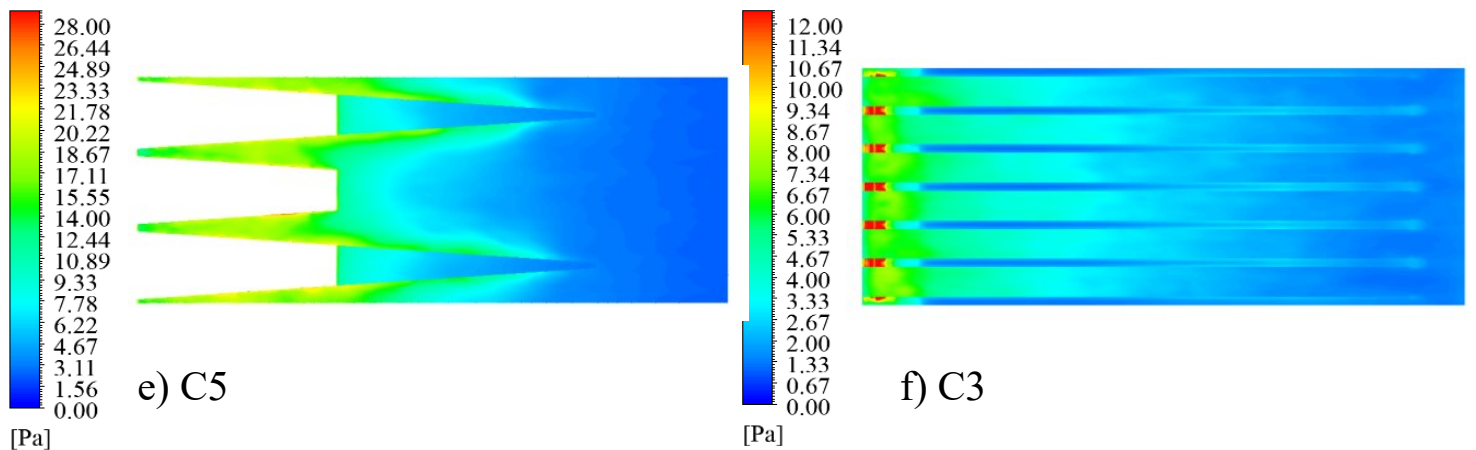

Figure 19: $P_{r m s}$ distribution on airfoil surface.
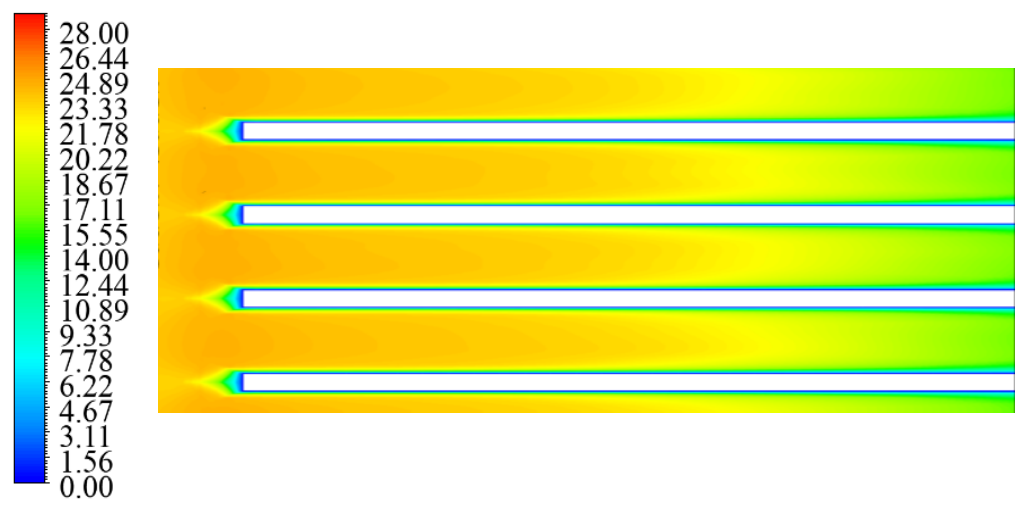

Figure 20: Time-averaged velocity contour (C3). 
The $P_{r m s}$ distribution indicates where the most violent pressure fluctuations occur. Identifying those locations is an important step for the applications of local aeroacoustic improvements to any TE design, since regions with the highest $P_{r m s}$ are likely to emit the most noise. Results show that the introduction of serrations concentrates the maximum pressure fluctuation region from the extremity of the TE to downstream of the serration roots. The serration amplitude and root bluntness affect the magnitude of pressure fluctuations, such that longer serrations cause stronger fluctuations. Slanted-root serrations decrease the $P_{r m s}$ relative to standard serrations and push its location further downstream. The introduction of TE finlets is shown to allocate the stronger pressure fluctuations close to the extremity of the finlets. The following subsection looks into the TBL as it is being convected downstream by means of TKE distribution.

\subsubsection{Turbulence Kinetic Energy Distribution}

The turbulence kinetic energy (TKE) of the flow is defined as the change in the velocity fluctuation, having the units $\mathrm{m}^{2} / \mathrm{s}^{2}$. The values of $k$ are obtained by directly solving the differential transport equations for the turbulence kinetic energy [70,71], as described in subsection 2.1. The instantaneous TKE distributions at

planes defined perpendicular to the free-stream direction are generated and displayed at locations of interest.

Figure 21 shows the TKE distribution for the case of a flat TE airfoil (C1.2). The TKE distribution remains undisturbed as the flow approaches the airfoil TE and the TBL grows in the streamwise direction. Figure 22 demonstrates the effect of standard serrations on the TKE distribution. Figure 22a shows the onset of mixing between the upper and lower airfoil sides for $\mathrm{C} 2.1$ at $x / c=0.95$. Figure $22 \mathrm{c}$ shows the same event taking place in $\mathrm{C} 2.2$, but earlier at $x / c=0.9$, because the serration amplitude is double $\left(2 h_{C 2.1}=30 \mathrm{~mm}\right.$ and $\left.2 h_{C 2.2}=60 \mathrm{~mm}\right)$. C2.1 shows a more uniform, less turbulent distribution compared to $\mathrm{C} 2.2$ and $\mathrm{C} 2.3$, 
which is an indication that longer serrations promote turbulence, and subsequently improve mixing. $\mathrm{C} 2.2$ and $\mathrm{C} 2.3$ exhibit similar behaviour at the same locations, as can be seen by comparing Figure 22c and 22e. At $x / c=1$ (Figures 22b, 22d and 22f), high TKE air surrounds the serration tips.

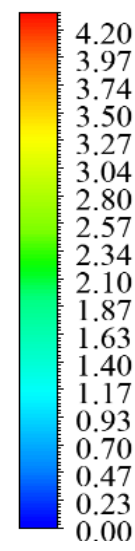

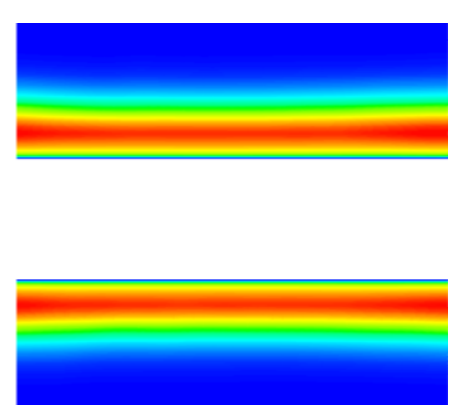

a) $\mathrm{C} 1.2$ at $x / c=0.9$

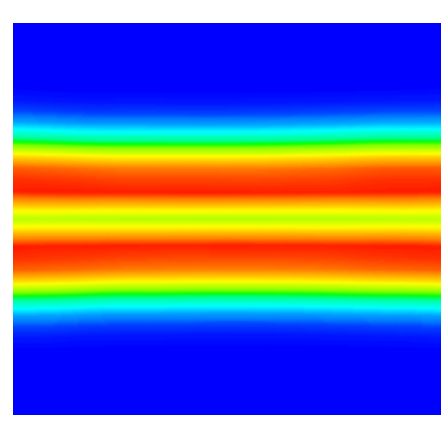

b) $\mathrm{C} 1.2$ at $x / c=1$

Figure 21: Instantaneous TKE distribution for a flat TE airfoil.

The effect of finlets on the instantaneous TKE distribution is shown in Figure 23. Air is channelled between the finlets; the initially undisturbed boundary layer is deformed and decorrelated, and the highly energetic fluid particles are kept away from the airfoil surface (Figures 23a to 23c), thus decreasing the efficiency of acoustic scattering $[95,96]$. Unlike in the case of serrations, the flow is not allowed to mix across the airfoil surface. The observed TKE magnitudes at $x / c=$ 1 are less than in all the previous cases. 


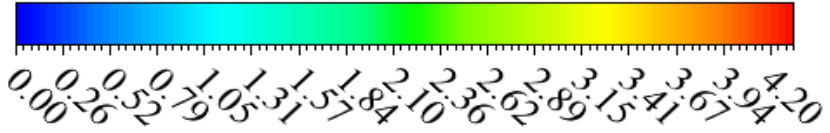

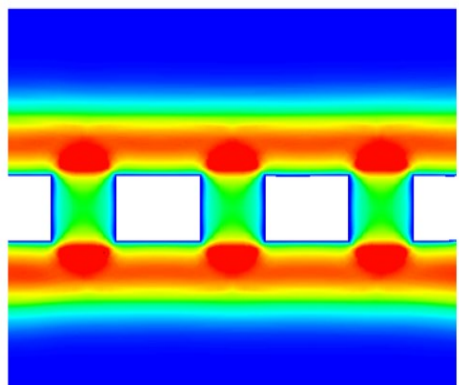

a) $\mathrm{C} 2.1$ at $x / c=0.95$

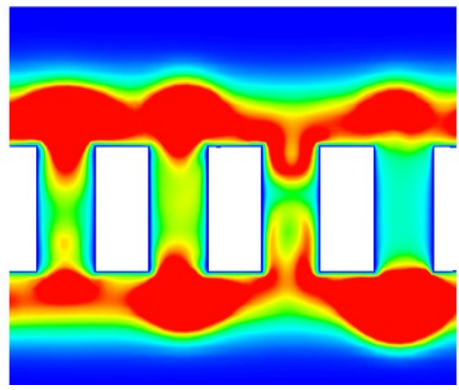

c) C2.2 at $x / c=0.9$

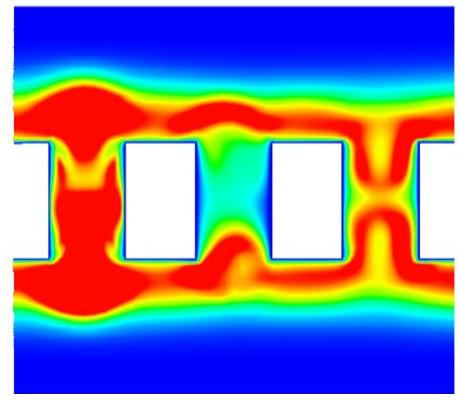

e) C2.3 at $x / c=0.9$

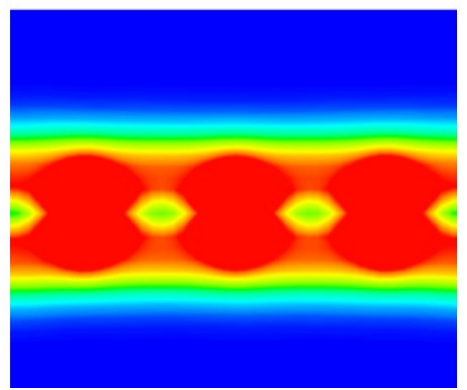

b) C2.1 at $x / c=1$

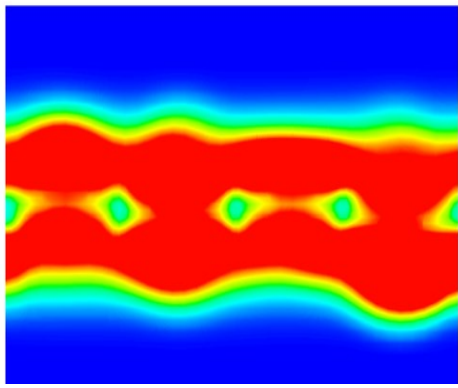

d) C2.2 at $x / c=1$

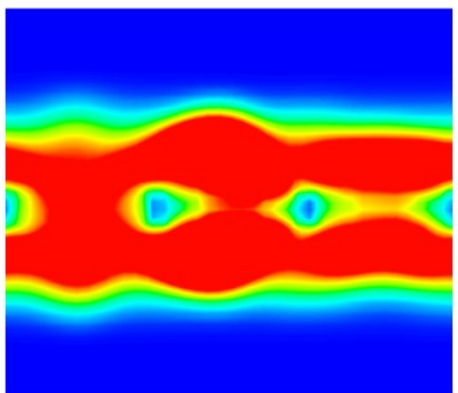

f) $\mathrm{C} 2.3$ at $x / c=1$

Figure 22: Instantaneous TKE distribution for standard TE serrations. 

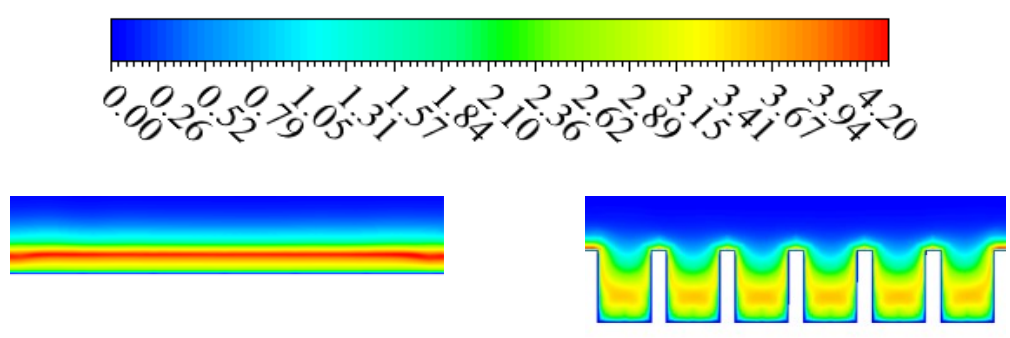

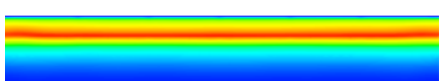

a) $\mathrm{C} 3$ at $x / c=0.73$

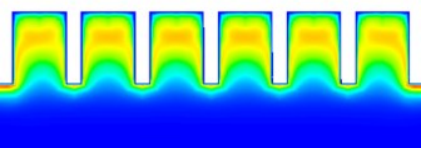

b) $\mathrm{C} 3$ at $x / c=0.9$

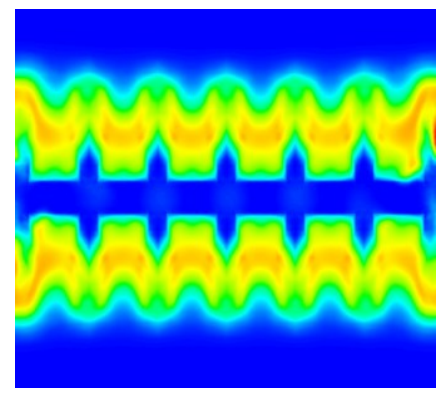

c) $\mathrm{C} 3$ at $x / c=1$

Figure 23: Instantaneous TKE distribution for TE finlets.

Figure 24 illustrates the effect of finned serrations on the instantaneous TKE distribution. The flow is progressively allowed to leak between the serrations as the introduced gaps get wider (Figure 24a), while being channelled by the finlets (Figure 24b). The TKE of the flow is higher than in the case of finlets (C3) and lower than the cases of standard serrations (C2.2 and C2.3). A phase shift observed between the flow channels, which is explained by the difference between the flow channels of C3 and C4, as seen in Figure 25. The surface of the finlets is coloured in gray for clarity. The flow channels in C3 are identical, unlike for C4. Instead of remaining convected away from the surface, like in case $\mathrm{C} 3$, the energetic eddies leak into the serration gaps and populate them. Flow channeling and decorrelation can be particularly useful in the design of low-noise airfoils, as proper phase control between the flow channels could be leveraged to induce destructive acoustic interference, which would reduce the generated noise. 


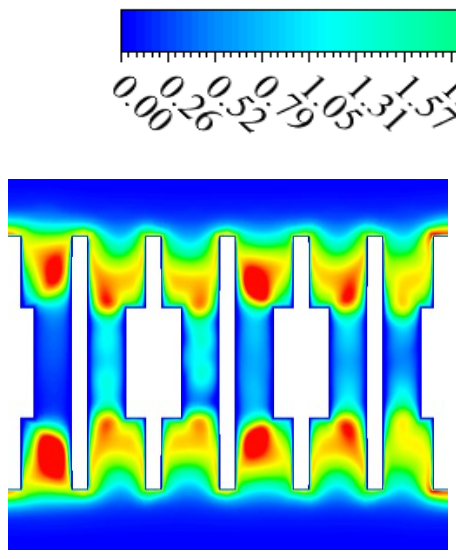

a) $\mathrm{C} 4$ at $x / c=0.9$

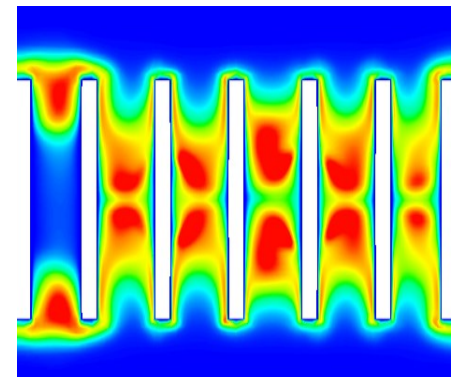

b) $\mathrm{C} 4$ at $x / c=0.96$

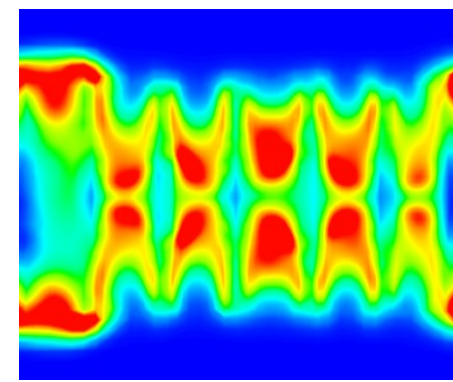

c) $\mathrm{C} 4$ at $x / c=1$

Figure 24: Instantaneous TKE distribution for finned TE serrations.

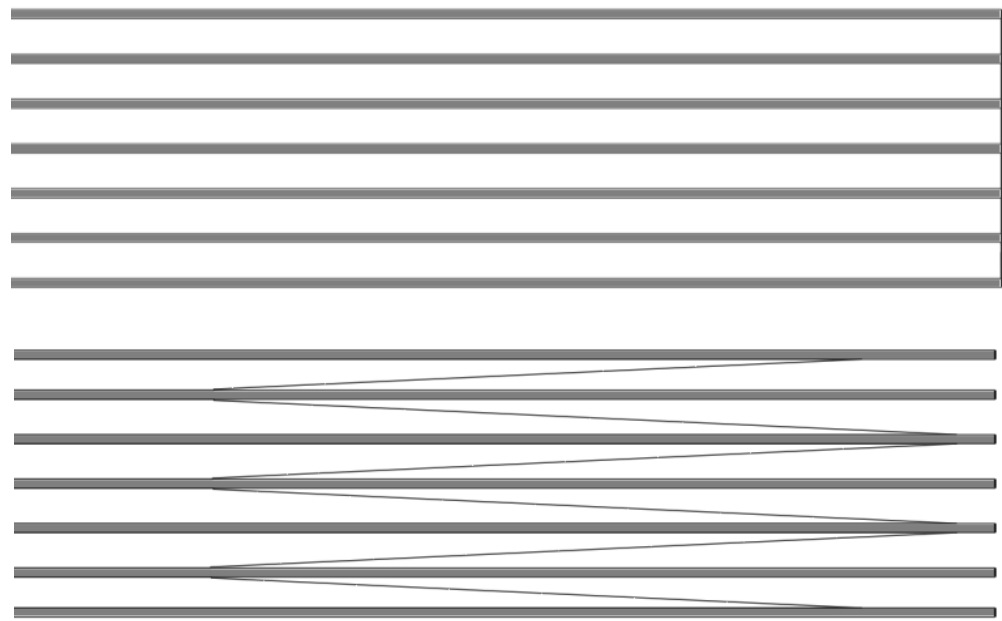

Figure 25: Difference in flow channels for cases C3 (top) and C4 (bottom). 
Figure 26 shows the effect of slanted-root serrations on the instantaneous TKE distribution. Figure 26a gives the TKE distribution right before the extremity of the slanted root. At $x / c=0.89$, the energetic flow particles progressively populate the introduced slanted slots as they grow. The flow is channeled between the slanted slots and the bluntness of standard serration roots is mitigated. This phenomenon is similar to the channeling effect of finlets seen in C3 and C4. Figure 26b shows the TKE distribution at $x / c=0.96$, where the flow is free to cross the gaps between the serrations. At $x / c=1$, the airfoil is exposed to less energetic fluid particles, compared to $\mathrm{C} 2.2$ and $\mathrm{C} 2.3$. This remark constitutes another resemblance between the effects of slanted-root serrations and finlets, where the energetic eddies are convected away from the airfoil surface to the reduce the TE-scattering efficiency.

Visualizing the instantaneous TKE distribution contours at different streamwise locations is helpful for understanding the behavior of the highlyenergetic, turbulent structures found in the BL. In the case of a flat TE (C1.2), the TKE distribution remains almost constant as the BL develops and the flow moves in the streamwise direction. Standard serrations (C2.1, C2.2 and C2.3) allow the energetic eddies to mix and move across the airfoil surface. Finlets (C3) channel the flow, lift the energetic eddies away from the airfoil surface and reduce the TKE near the airfoil TE. Finned serrations (C4) channel the flow while allowing it to mix between the serrations. Slanted-root serrations initially channel the flow between the introduced slots $(x / c \leq 0.9)$ then allow it to mix as it reaches the edge of the slanted root. 


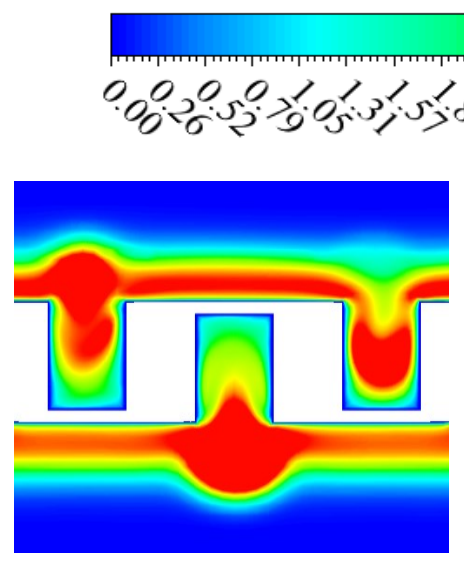

a) C5 at $x / c=0.89$

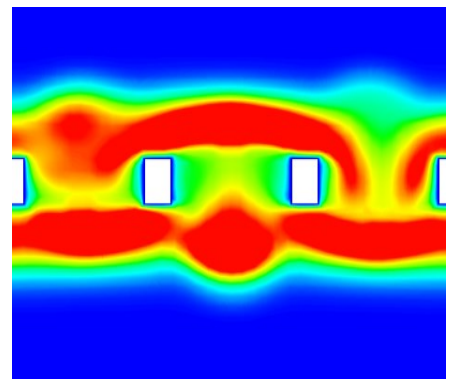

b) $\mathrm{C} 5$ at $x / c=0.96$

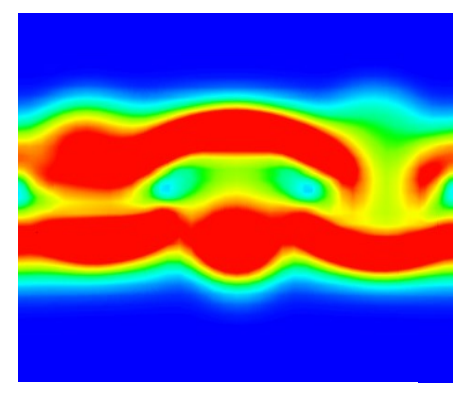

c) $\mathrm{C} 5$ at $x / c=1$

Figure 26: Instantaneous TKE distribution for slanted-root TE serrations.

\subsubsection{Wake Vorticity and Characteristics}

By calculating the coefficient of lift, $c_{L}=\frac{L}{\frac{1}{2} \rho v^{2} A}$, for every timestep of the flow simulation, the lift-coefficient history can be plotted. $L$ is defined as the lift force and $A$ is the airfoil area. The lift-coefficient history is commonly used as an indicator of statistical convergence in transient simulations. Furthermore, it's a nondimensional representation of the fluctuating forces acting normal to the airfoil surface. Figure 28 presents the lift-coefficient history for cases C1.2, C2.1, C2.2 and C2.3, as well as an overlay plot of all the cases. All axes are kept constant and aligned for clarity and comparison. As expected, for C1.2 the lift-coefficient history 
appears random and irregular, characterized by a relatively small amplitude, which is a sign of broadband behavior. With the introduction of standard serrations, the lift monitors adopt sinusoidal profiles, each dominated by a single frequency. The underlying frequency for every case is calculated by taking the FFT of the timehistory signals. C2.1 is subject to the highest frequency $(388 \mathrm{~Hz})$ and smallest amplitude, while $\mathrm{C} 2.2$ is subject to the lowest frequency $(218 \mathrm{~Hz})$. The amplitude is the same for C2.2 and C2.3, but the frequency is higher in C2.3 (248 Hz). The lift-coefficient history amplitudes are higher in all standard-serration cases when compared to the case of a flat TE, which means the airfoil is experiencing stronger fluctuating forces.

In order to demonstrate the convergence of the computational mesh, C2.3 was simulated twice using two different meshes having 6,624,133 and 7,224,158 elements, respectively. The resulting lift-coefficient history plots are shown in Figure 27. Both simulations yield the same lift-variation amplitude and frequency once the flow is initialized. The only observable difference is a phase shift, which is normal and means each simulation started from a different point in the periodic cycle. The observed common amplitude and variation frequency prove mesh convergence and show that the used mesh is fine enough to accurately resolve the flow, and further refinement of the mesh will not affect hydrodynamic results of interest.

Figure 29 shows the effect of finlets (C3) and finned serrations (C4) relative to a flat TE (C1.2). The behavior of $\mathrm{C} 3$ doesn't significantly change from $\mathrm{C} 1.2$, in terms of the random broadband fluctuations and their amplitudes, which means the fluctuating forces the airfoil itself is subject to with a finned TE (C3) are almost the same as for a clean one (C1.2). In $\mathrm{C} 4$, sinusoidal behavior is observed, similarly to standard serrations. C2.2, C2.3 and C4 share the same serration amplitude, $2 \mathrm{~h}=60$ $\mathrm{mm}$, and root bluntness, $\epsilon=16.3 \mathrm{~mm}$. The amplitude of variation of lift coefficient is less in $\mathrm{C} 4$ than in $\mathrm{C} 2.2$ and $\mathrm{C} 2.3$, which is considered an improvement as the airfoil experiences weaker fluctuating lift forces. The lift-coefficient variation frequency is equal to $210 \mathrm{~Hz}$. The observed sinusoidal behaviors are signs of vortex shedding in some of the airfoils. 
Figure 30 illustrates the lift-coefficient history of slanted-root serrations (C5) relative to standard serrations ( $\mathrm{C} 2.1$ and $\mathrm{C} 2.2)$. Since $\mathrm{C} 2.2$ is similar to $\mathrm{C} 2.3$, $\mathrm{C} 2.3$ was not plotted in the interest of clarity. Slanted-root serrations exhibit a unique behavior when compared to standard serrations, as well as all the other cases. The lift-coefficient history is no longer a clearly defined sinusoidal wave dominated by a single frequency. Instead, it's showing a more random, quasibroadband behavior. Furthermore, the accompanying fluctuating forces are less than in $\mathrm{C} 2.2$ and $\mathrm{C} 2.3$, as the lift variation amplitude is significantly reduced. This is a strong indicator of the deterioration of vortex shedding.

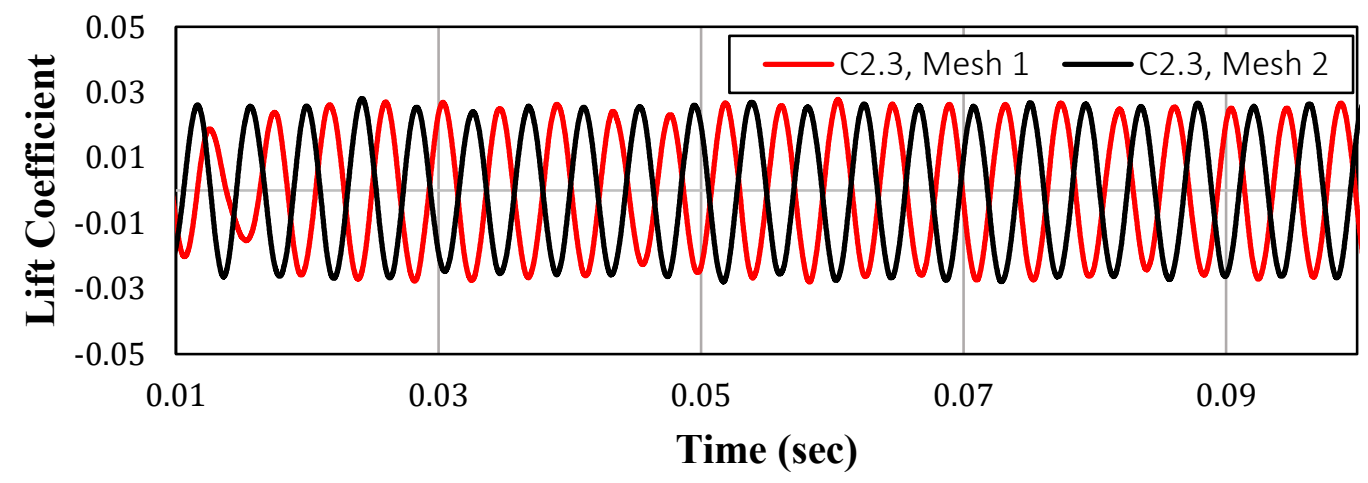

Figure 27: Lift-coefficient history of $\mathrm{C} 2.3$ using two different meshes. 

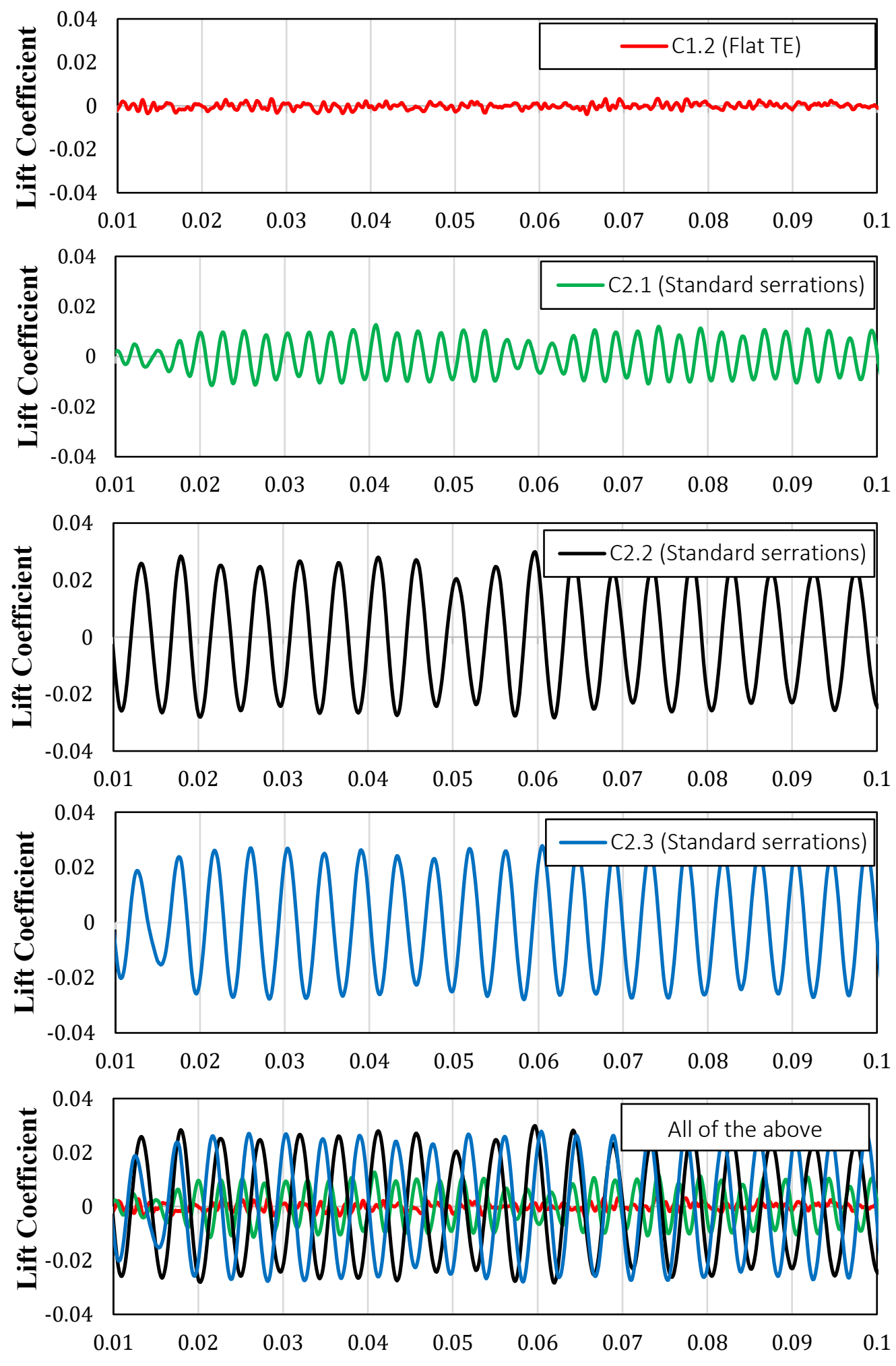

Time (sec)

Figure 28: Lift-coefficient history for C1.2, C2.1, C2.2 and C2.3. 

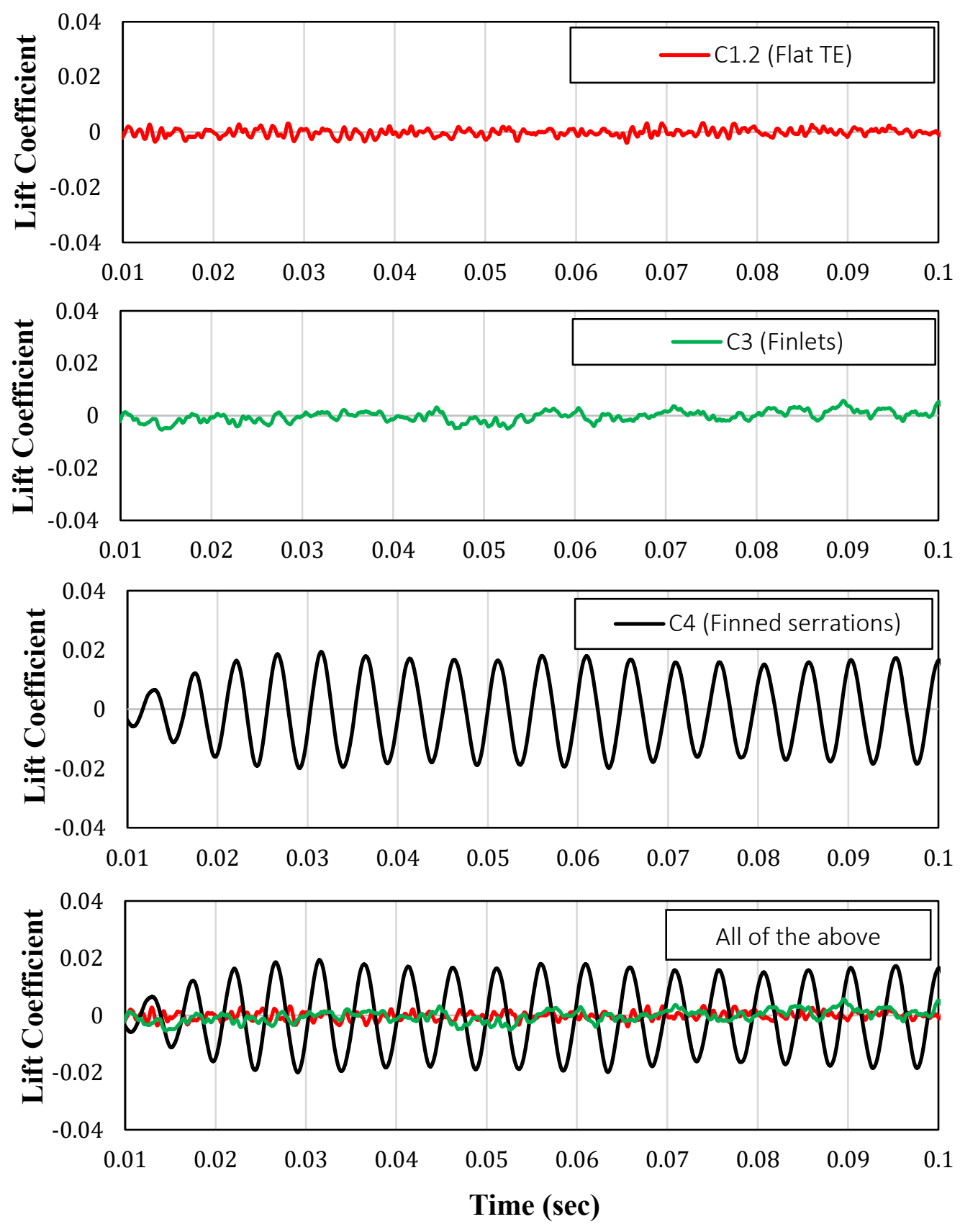

Figure 29: Lift-coefficient history for $\mathrm{C} 1.2, \mathrm{C} 3$ and $\mathrm{C} 4$. 

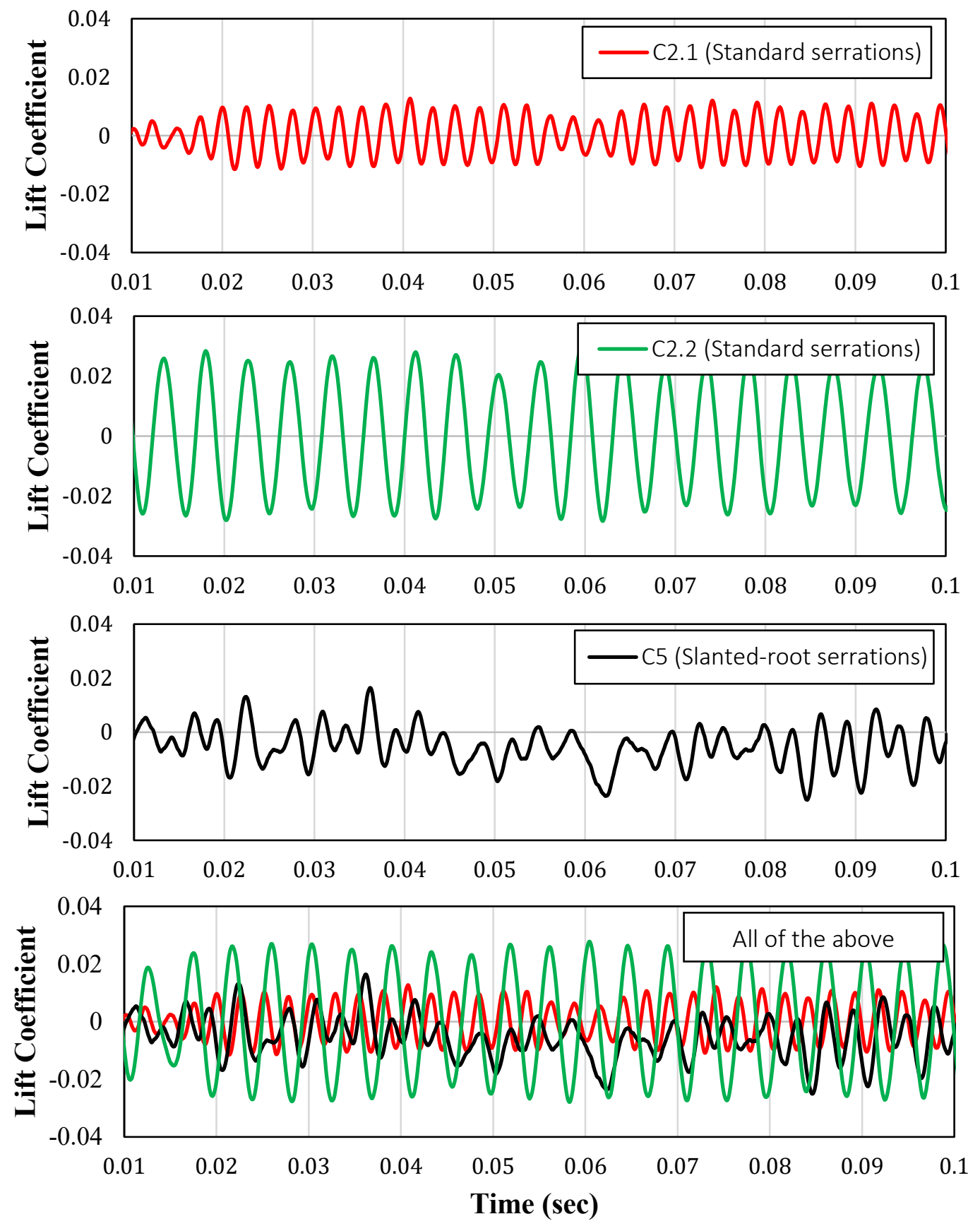

Figure 30: Lift-coefficient history for $\mathrm{C} 2.1, \mathrm{C} 2.2$ and $\mathrm{C} 5$.

The evolution and diffusion of TE vorticity can be illustrated using contours of iso-vorticity, where vorticity is defined as the curl of velocity, or two times the angular velocity, and thus having the units $\mathrm{s}^{-1}$. Vorticity describes how the infinitesimal units of flow rotate as they travel in the free-stream direction. 
Figure 31 presents the wake vorticity magnitude for all the simulated cases at planes normal to the $z$-axis, where the $z$-axis points in the spanwise direction. For a flat TE (C1.2), wake vorticity is non-uniform and has almost no observable coherent structures. Vorticity is strongest near the TE and is gradually weakened (i.e. dissipated) as air is convected in the streamwise direction. For all the cases of standard serrations (C2.1, C2.2 and C2.3), the contours reveal sinusoidal patterns having different wavelengths and frequencies, which means that alternating low pressure zones are shed from the TE, resulting in vortex shedding. $\mathrm{C} 2.1$ is subject to the smallest amplitude and highest frequency. C2.2 and C2.3 are subject to comparable oscillation amplitudes but different frequencies, such that the oscillation frequency is higher is $\mathrm{C} 2.3$.

In the case of finlets (C3), vorticity in the wake is similar to C1.2, but the vortical region upstream of the airfoil TE is stronger (31a and 31e). Finned serrations (C4) experience a sinusoidal pattern similar to the one observed in standard serrations, but the oscillations start further downstream (31c and 31f). Slanted-root serrations (C5) experience weaker vorticity and a smaller oscillation amplitude when compared to C2.3 (31d and 31g). 

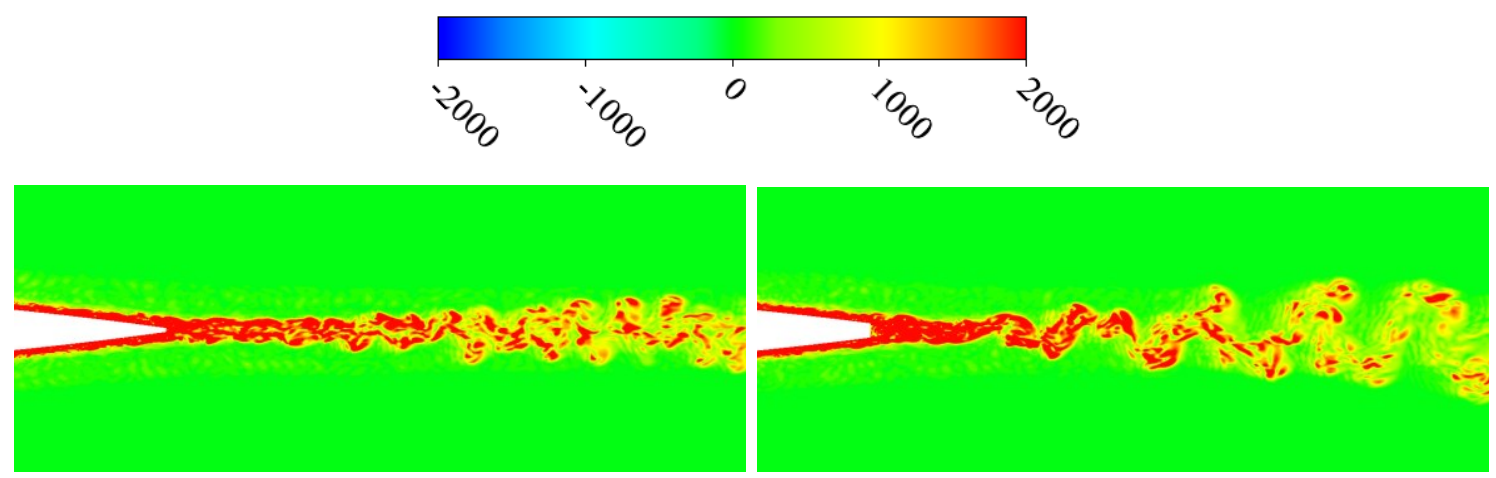

a) $C 1.2$ at $z / s=0.5$

b) $\mathrm{C} 2.1$ at $z / \mathrm{s}=0.5$
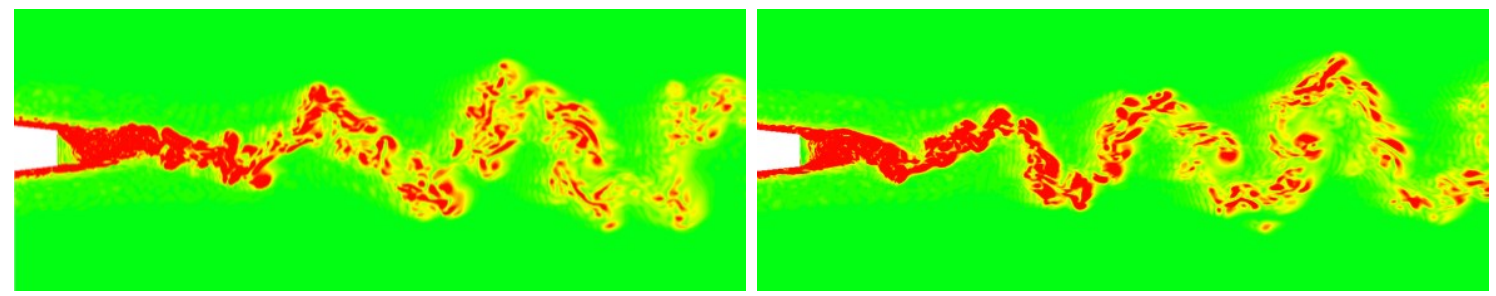

c) $\mathrm{C} 2.2$ at $\mathrm{z} / \mathrm{s}=0.625$

d) $\mathrm{C} 2.3$ at $z / \mathrm{s}=0.5$
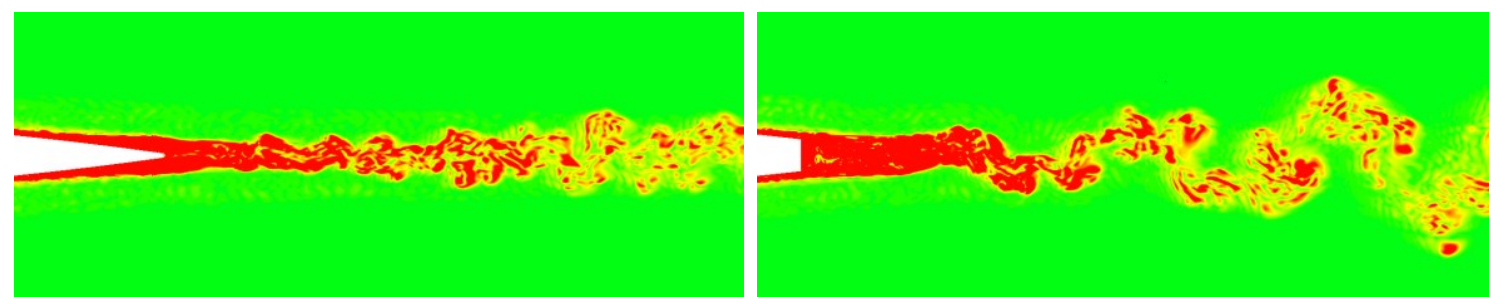

e) $\mathrm{C} 3$ at $z / s=0.5$

f) $\mathrm{C} 4$ at $z / s=0.53$

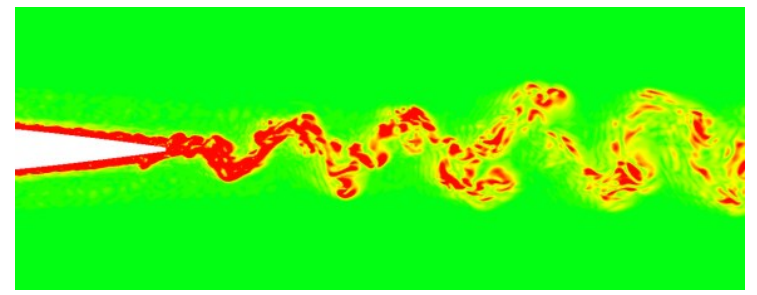

g) $\mathrm{C} 5$ at $z / s=0.33$

Figure 31: Instantaneous wake vorticity magnitude.

To further investigate the variation of the observed vortical structures, it is important to identify and portray the desired turbulent coherent structures, which are inherently three-dimensional. The Q-Criterion, initially proposed by Hunt et al. [97], and later defined by Chong et al [98] as the second invariant of the instantaneous velocity gradient tensor, is one of the most widely used methods for 
the classification of such structures [99]. Figure 32 shows the instantaneous vortical structures in terms of the Q-Criterion in the wake of the simulated airfoils, where the value of $Q$ is set to 0.01 . The value of spanwise vorticity, $\omega_{z}$, is expressed in colour gradation on the $\mathrm{Q}$ iso-surfaces. For the case of a flat TE $(\mathrm{C} 1.2)$, the wake is turbulent but has no identifiable coherent structures. The absolute value of $\omega_{z}$ decreases in the streamwise direction, which is expected for a symmetric NACA0012 airfoil since it does not generate lift (net vorticity=0).

For the cases of standard serrations (C2.1, C2.2 and C2.3), sinusoidal vortex shedding is observed and confirmed. The amplitude and frequency of the observed phenomenon change as the serrations amplitude and wavelengths are varied. The vortex shedding frequency is, by definition, equal to the lift variation frequency. This means C2.1 is subject to the highest shedding frequency $(388 \mathrm{~Hz}), \mathrm{C} 2.2$ the lowest shedding frequency $(218 \mathrm{~Hz})$, and C2.3 falls in between $(248 \mathrm{~Hz})$. The vortex shedding frequency can also be approximated by counting the number of full periodic cycles found in the airfoil wake and dividing it by the through-flow time $\left(T_{T F}\right)$ of the simulation domain. The vortical structures seen in C2.1, C2.2 and C2.3 are dissipated earlier in the wake when compared to C1.2. The vortex shedding amplitudes are equal for C2.2 and C2.3, and the amplitude is smallest for C2.1.

Vortex shedding is caused by the interaction of two shear layers as they leave the airfoil surface. Because of the inherent instability of the TBL, alternating low pressure zones are generated downstream of the airfoil, giving rise to fluctuating forces acting normal to the wind direction, which in turn explains the sinusoidal lift coefficient variation (Figure 28). A generated vortex is initially growing and fed by circulation from the separated shear layer, until it becomes strong enough to roll up and draw the opposing shear layer across the wake. At that point, this vorticity of opposite sign interrupts any further supply of circulation to the growing vortex, which then stops increasing in strength. As a result, that vortex is shed and convected downstream while a new one of opposite vorticity takes its place and the cycle keeps going [100,101]. As the serration amplitude (2h) is reduced, the root bluntness $(\epsilon)$ is also reduced and the shear layers are brought closer together. Subsequently, the interaction between the two shear layers is 
facilitated and the periodic time is shortened, giving rise to a higher vortex shedding frequency [101]. Radiated tonal peaks are fundamentally caused by the observed vortex shedding [102].

The wake created by TE finlets (C3) is random, similar to what's observed in the case of a flat TE (C1.2). Looking at the region upstream of the TE in Figure $32 \mathrm{e}$, it is apparent that the region of increased vorticity, previously seen in Figure $31 \mathrm{e}$, is defined by the height and profile of the finlets themselves. Downstream of the TE, the wake is irregular and similar to C1.2. No vortex shedding is observed. Finned serrations (C4) give rise to sinusoidal vortex shedding, the onset of which is delayed when compared to the cases of standard serrations. The vortex shedding amplitude is smaller than in $\mathrm{C} 2.2$ and $\mathrm{C} 2.3$. The observed vortices become less defined as they are convected in the streamwise direction. $\mathrm{C} 4$ is subject to a vortex shedding frequency of $210 \mathrm{~Hz}$. The dynamics of vortex shedding are the same as previously described for standard serrations.

The wake of slanted root serrations (C5) is characterized by weaker, less defined vortices when compared to C2.2 and C2.3. The observed sinusoidal distributions lose definition as the flow moves downstream, which means the shed vortices are dissipated quicker as they are convected downstream of the TE. The shedding amplitude is also less than in $\mathrm{C} 2.2$ and $\mathrm{C} 2.3$, which agrees with the observed lift-coefficient history behavior and the reduction in the maximum surface $P_{r m s}$ values. 

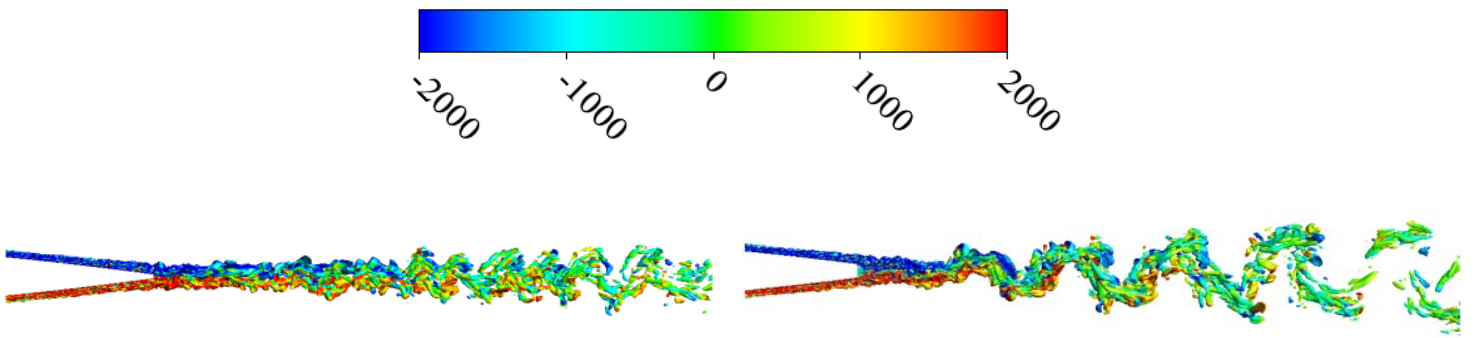

a) $\mathrm{C} 1.2$

b) $\mathrm{C} 2.1$
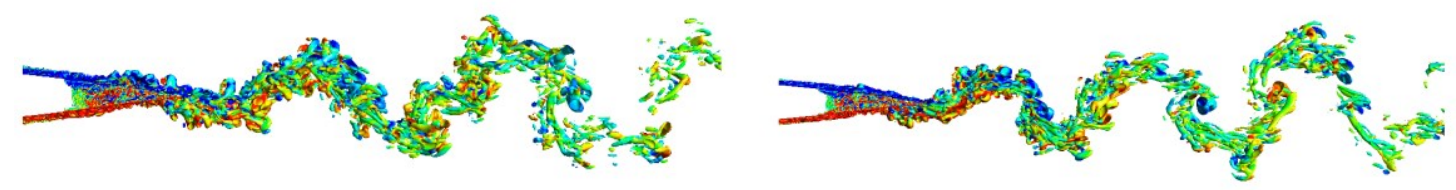

c) $\mathrm{C} 2.2$

d) $\mathrm{C} 2.3$

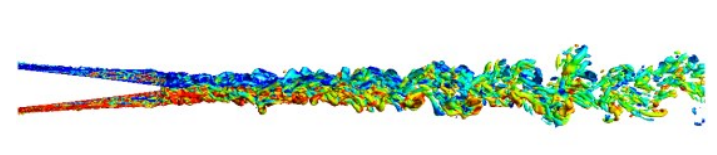

e) $\mathrm{C} 3$

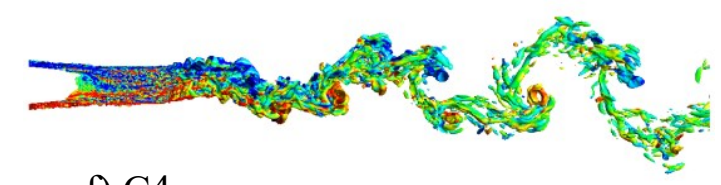

f) $\mathrm{C} 4$

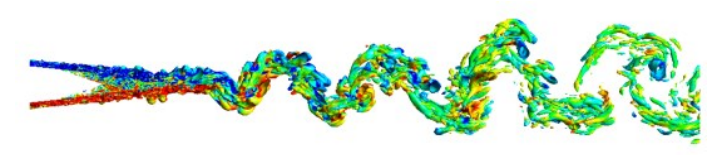

g) $\mathrm{C5}$

Figure 32: Instantaneous Q-Criterion coloured by $\boldsymbol{\omega}_{\mathbf{z}}$.

Lastly, Figure 33 gives the streamwise vorticity, $\omega_{x}=\frac{\partial u_{y}}{\partial z}-\frac{\partial u_{z}}{\partial y}$, contours for all the simulated cases. The two limits of the contour correspond to fluid particles having equal vorticity but in opposite directions. For the case of a flat TE (C1.2), turbulent, counter-rotating coherent structures are observed at the TE. The turbulent structures are not allowed no cross the airfoil surface. Because information cannot travel across the upper and lower airfoil surfaces, the two TBLs are completely independent until the instant at which they collide right after the TE. This "discontinuity" is responsible for the majority of the radiated noise $[5,95]$. 
For cases having standard serrations (C2.1, C2.2 and C2.3), the instantaneous $\omega_{x}$ contours shown in Figures 33b to 33d demonstrate the existence of counterrotating vortical structures that are permitted to pass between the serrations. The flow on opposite sides of the airfoil is allowed to progressively mix as it is convected in the streamwise direction, thus mitigating the otherwise sudden discontinuity encountered at the sharp TE of a clean airfoil.

Finlets (C3) are shown to channel the turbulent coherent structures between the finlets. No mixing is observed as the fluid cannot cross the solid airfoil, but the size of the coherent structures is smaller than in all the aforementioned cases. The size of these structures can be manipulated by the changing the spacing between the finlets, S. Smaller spacing would lead to smaller turbulent structures that radiate a higher frequency noise, while coarser spacing would lead to larger structures radiating lower frequency noise [30]. If the spacing becomes too fine, there is a risk of the finlets behaving like a solid object, where the majority of flow would go over the finlets instead of through them [30].

Finned serrations (C4) channel the turbulent counter-rotating eddies between the finlets and reduce their size, as isolated finlets would. In addition, the flow structures on the opposite sides of the airfoil surface are allowed to mix as they would in the case in standard serrations. Figure 33g shows the counter-rotating vortical structures present near the airfoil TE in C5. Flow structures are allowed to mix and move across the airfoil. Slanted-root serrations promote streamwise vorticity, $\omega_{x}$, and improve mixing across the airfoil, as standard serrations would. 


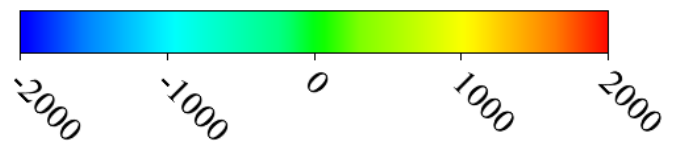

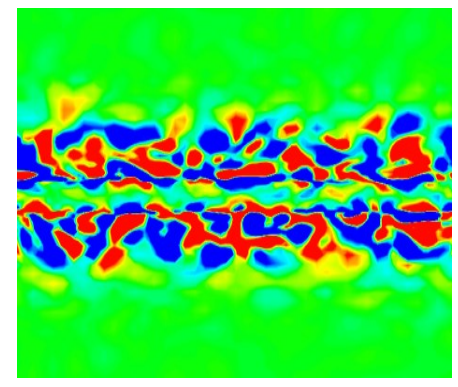

a) $\mathrm{C} 1.2$

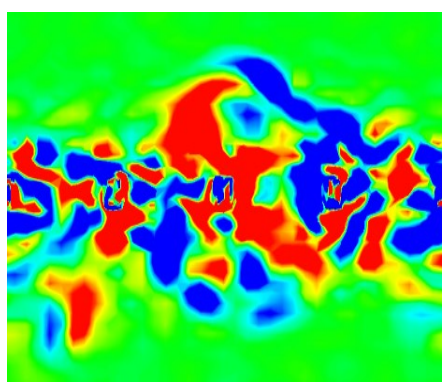

c) $\mathrm{C} 2.2$

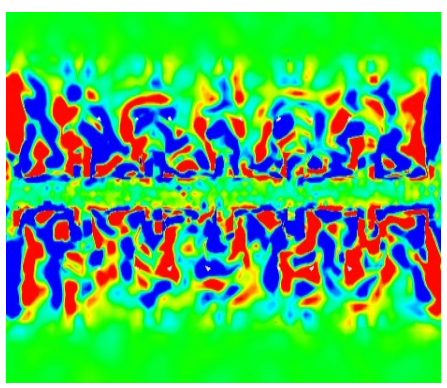

e) $\mathrm{C} 3$

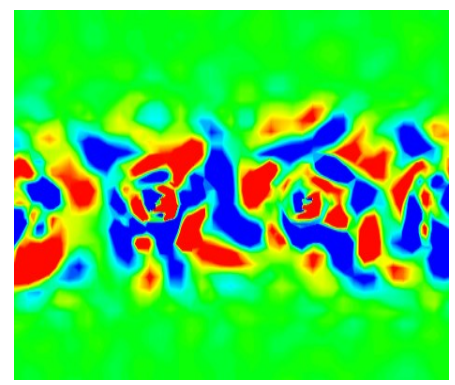

b) $\mathrm{C} 2.1$

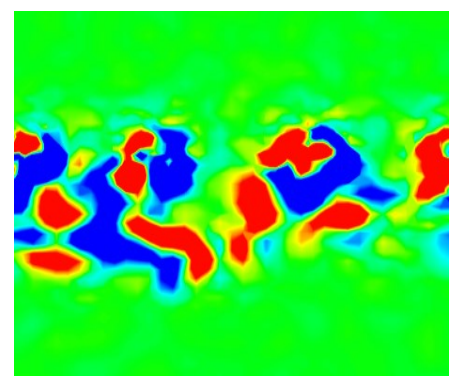

d) $\mathrm{C} 2.3$

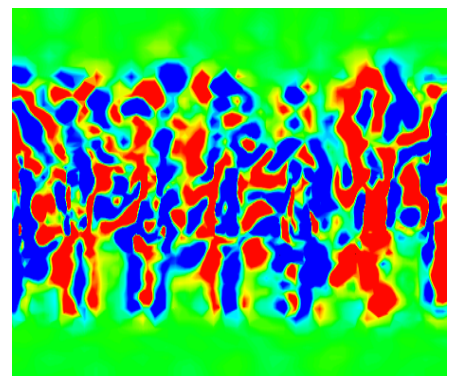

f) $\mathrm{C} 4$

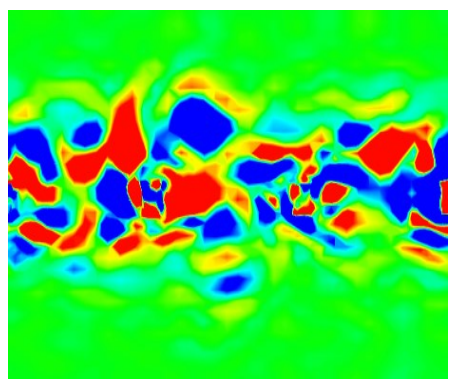

g) $\mathrm{C} 5$

Figure 33: Instantaneous $\omega_{x}$ at $x / c=1$. 
In summary, the lift-coefficient history plots of all the simulated cases are shown. C1.2 and C3 are subject to random, broadband lift variations. C2.1, C2.2, C2.3 and C4 show sinusoidal fluctuations. C5 gives a unique behavior that is not a pure sine wave, but not as broadband as C1.2 and C3. The vorticity magnitude in the wake is looked into. C1.2 and C3 show a non-uniform vorticity distribution in the wake. C2.1, C2.2, C2.3 and C4 are subject to sinusoidal vorticity distributions, having different wavelengths and amplitudes. C5 is characterized by less defined vorticity downstream of the TE, compared to C2.3. Vortex shedding is observed when inspecting the Q-Criterion iso-surfaces of C2.1, C2.2, C2.3 and C4. C5 is also subject vortex shedding but it appears weaker and loses definition earlier in the downstream direction. Cases C2.1, C2.2, C2.3 and C4 are subject to vortex shedding frequencies of $388 \mathrm{~Hz}, 218 \mathrm{~Hz}, 248 \mathrm{~Hz}$ and $210 \mathrm{~Hz}$, respectively. Instantaneous $\omega_{x}$ contours are used to investigate mixing at the TE of all the simulations. The flow is allowed to mix through the airfoil surface and fill the gaps introduced by serrations in C2.1, C2.2, C2.3 and C4. Finally, C3 and C4 are characterized by the smallest coherent structures when compared to all other cases. The following subsection presents the obtained far-field noise results.

\subsubsection{Far-field Noise}

The FW-H aeroacoustic analogy [8] is used to compute the radiated far-field noise for all simulated cases. Pressure fluctuations are propagated to receivers placed midspan at a distance of 1.5 meters directly above the airfoils' TEs. To obtain the time signal corresponding to every case, acoustic data is sampled every 2 flow-timesteps and data sampling is performed for $3 T_{F T}$ after the flow is fully developed, resulting in a sampling frequency of $41.67 \mathrm{kHz}$ and a frequency resolution of $28.4 \mathrm{~Hz}$, where the frequency resolution is defined as the inverse of the sampling period. Figure 34 shows all the resulting time signals. 

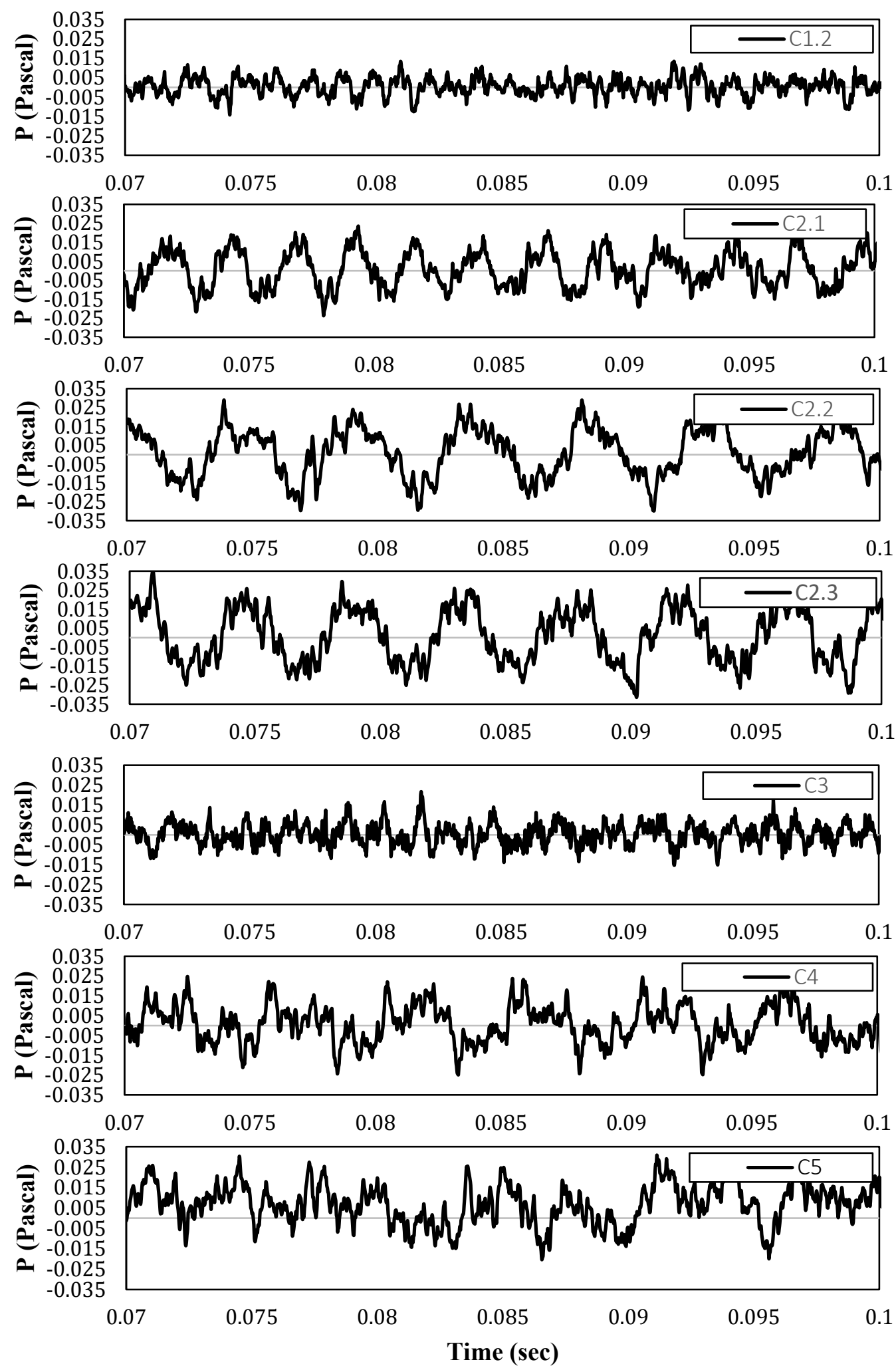

Figure 34: Time-domain pressure signals. 
Cases C2.1, C2.2 and C2.3 show periodic patterns, where the amplitude is highest in $\mathrm{C} 2.2$ and $\mathrm{C} 2.3$, and the periodic frequency is highest in $\mathrm{C} 2.1$. C1.2 and $\mathrm{C} 3$ are subject to similar, irregular signals. The observed pressure fluctuations in $\mathrm{C} 4$ are stronger than in $\mathrm{C} 3$, but weaker than in $\mathrm{C} 2.2$ and $\mathrm{C} 2.3$. A periodic trend is apparent, but the periodic cycles are not as defined as they are in $\mathrm{C} 2.2$ and $\mathrm{C} 2.3$. C5 demonstrates a behavior that is similar to that $\mathrm{C} 1.2$ and $\mathrm{C} 3$, such that no clearly identifiable periodic patterns are observed, unlike in C2.1, C2.2 and C2.3. The variation amplitude is larger than in $\mathrm{C} 1.2$ and $\mathrm{C} 3$.

Discrete FFT is applied on the time signals seen in Figure 34 to compute the sound pressure level (SPL) signal in the frequency domain. The Hanning window is applied to the time signal to reduce numerical leakages associated with the FFT [88]. Figure 35 shows the frequency-domain signals for all the cases of standard serrations (C2.1, C2.2 and C2.3) relative to the straight TE (C1.2). A narrowband tonal peak is observed in the spectrum of C2.1 at $397 \mathrm{~Hz}$. Similarly, tonal peaks are observed at $198 \mathrm{~Hz}$ for $\mathrm{C} 2.2$ and $240 \mathrm{~Hz}$ for C2.3. The tonal peak amplitudes are equal for $\mathrm{C} 2.2$ and $\mathrm{C} 2.3$. The peak amplitude is $3.3 \mathrm{~dB}$ lower in $\mathrm{C} 2.1$, meaning the tonal noise is louder for the cases having longer serrations. Figure 36 shows the acoustic spectra of case C2.3 obtained using 2 different meshes, as previously discussed. Both simulations predict comparable far-field noise, and the observed tonal peak amplitudes are equal and occur at the same frequency, $240 \mathrm{~Hz}$.

Figure 37 shows the acoustic spectrum of $\mathrm{C} 3$ relative to $\mathrm{C} 1.2$. $\mathrm{C} 3$ yields a broadband spectrum similar to that of C1.2. No major discrepancies are seen in terms of general trend and no tonal peaks are present. A band-pass filter was applied obtained signals for $\mathrm{C} 1.2$ and $\mathrm{C} 3$ to calculate the overall sound pressure level (OASPL) for different frequency ranges. An OASPL reduction of $0.488 \mathrm{~dB}$ was predicted in the frequency range $1000 \mathrm{~Hz}$ to $5000 \mathrm{~Hz}$.

Figure 38 shows the frequency-domain signal for the case of finned serrations (C4) relative to finlets (C3) and a case of standard serrations (C2.3). A narrowband tonal peak, similar to the ones observed in the cases of standard serrations, is predicted at $197 \mathrm{~Hz}$. The amplitude of tonal peak observed in C4 is 
$5.5 \mathrm{~dB}$ lower than in $\mathrm{C} 2.3,5.5 \mathrm{~dB}$ lower than in $\mathrm{C} 2.2$ and $2.2 \mathrm{~dB}$ lower than in $\mathrm{C} 2.1$. The tonal peak is generated by the vortex shedding that accompanies the introduced bluntness of the serration roots [102]. The weaker tonal noise is justified by the weakening of vortex shedding. It is hypothesized that flow spanwise decorrelation and the phase shift that was introduced between the flow channels are responsible for this weakening, and subsequently for the attenuation of the tonal peak. Finned serrations are shown to successfully combine the aerodynamic flow mechanisms of having finlets and standard serrations, in terms of flow decorrelation and mixing, respectively. By changing the spacing and thickness of finlets, relative to the amplitude and wavelength of serrations, tonal noise can be reduced and its frequency can be controlled. Results suggest that the coupling of different noisesuppressing mechanisms is a promising path to explore, with the goal of designing and developing new, quieter TE configurations. More research is still required to ensure proper coupling of the parameters of finlets and serrations, to induce a pronounced broadband noise reduction.

Figure 39 demonstrates the effect of slanting the serration roots on the radiated noise. The signal of $\mathrm{C} 5$ is plotted relative to $\mathrm{C} 2.1, \mathrm{C} 2.2$ and $\mathrm{C} 2.3$. Slantedroot serrations (C5) exhibit a significantly smoother acoustic spectrum, compared to standard serrations (C2.1 and C2.3), demonstrating a more broadband behavior that is free of narrow-band peaks. The tonal peaks observed in C2.1, C2.2 and C2.3 are reduced by $3.7 \mathrm{~dB}, 7 \mathrm{~dB}$ and $7 \mathrm{~dB}$, respectively. With the introduction of slanted-root serrations, the flow is no longer symmetrical about the $\mathrm{z}-x$ plane, which destabilizes the wake and facilitates the dissipation of the alternating low-pressure zones, which in turn weakens the vortex shedding. The vortices shed from slantedroots are weaker and less defined compared to those shed from standard roots, which leads to a more broadband noise source. The FW-H model successfully captured the suppression of the narrowband tonal peak traditionally observed in non-flat plate type serrations. Results of the numerical simulation agree with the observations of Chen et al. [28] and were presented in 50th International Congress and Exposition on Noise Control Engineering [103]. 


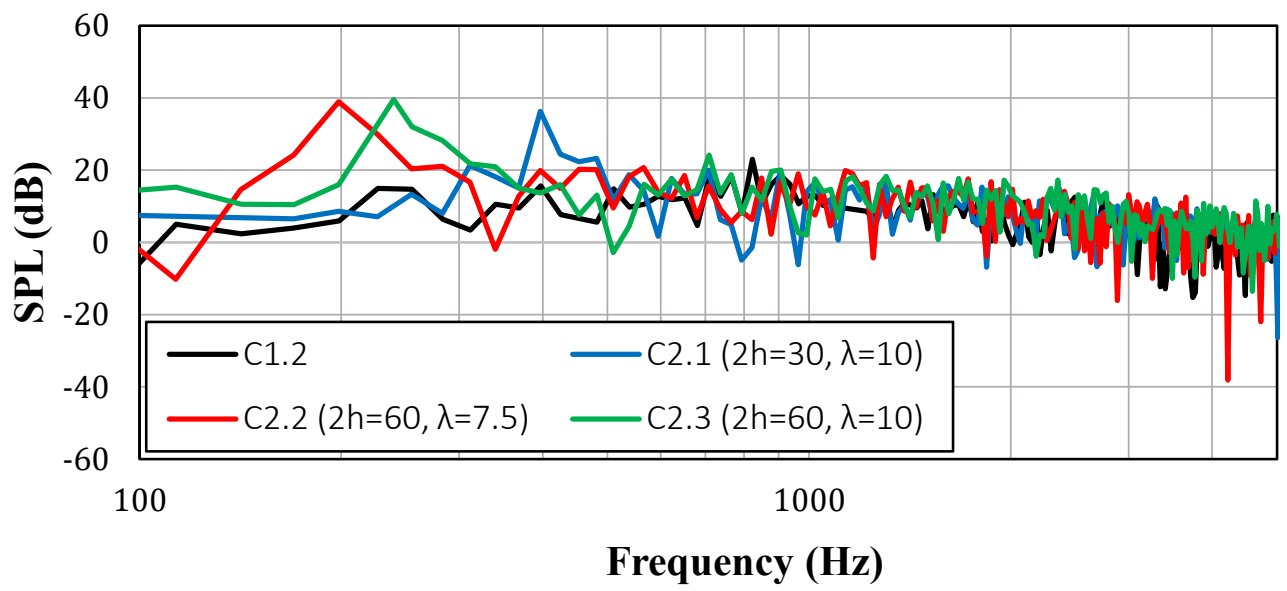

Figure 35: Frequency-domain noise signals of C1.2, C2.1, C2.2 and C2.3.

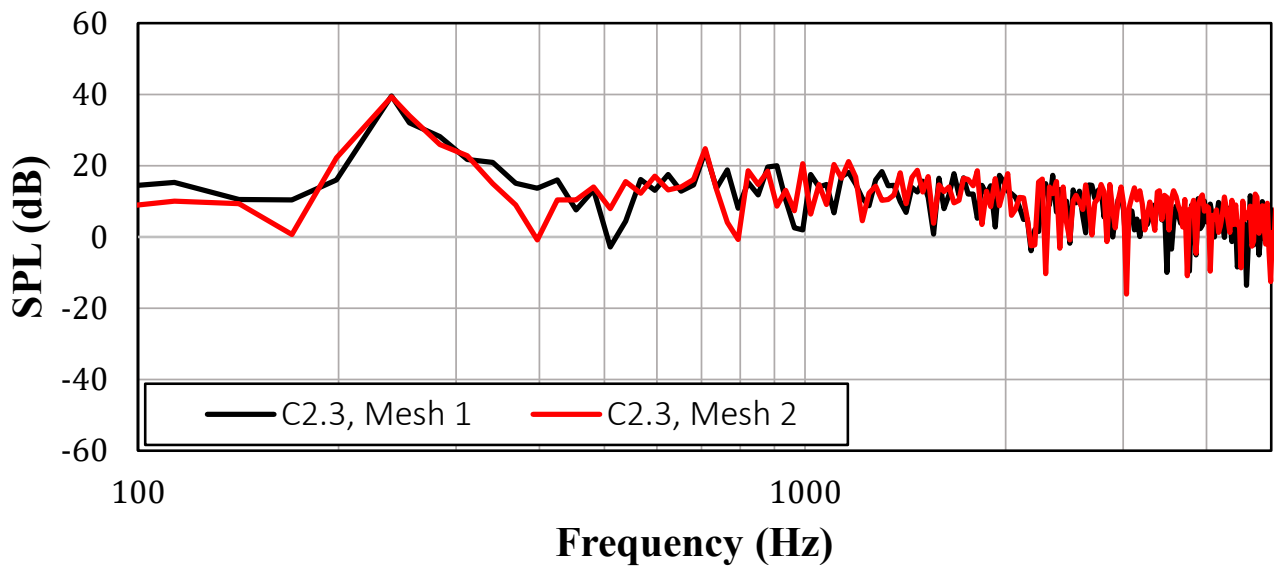

Figure 36: Frequency-domain noise signals of $\mathrm{C} 2.3$ using different meshes.

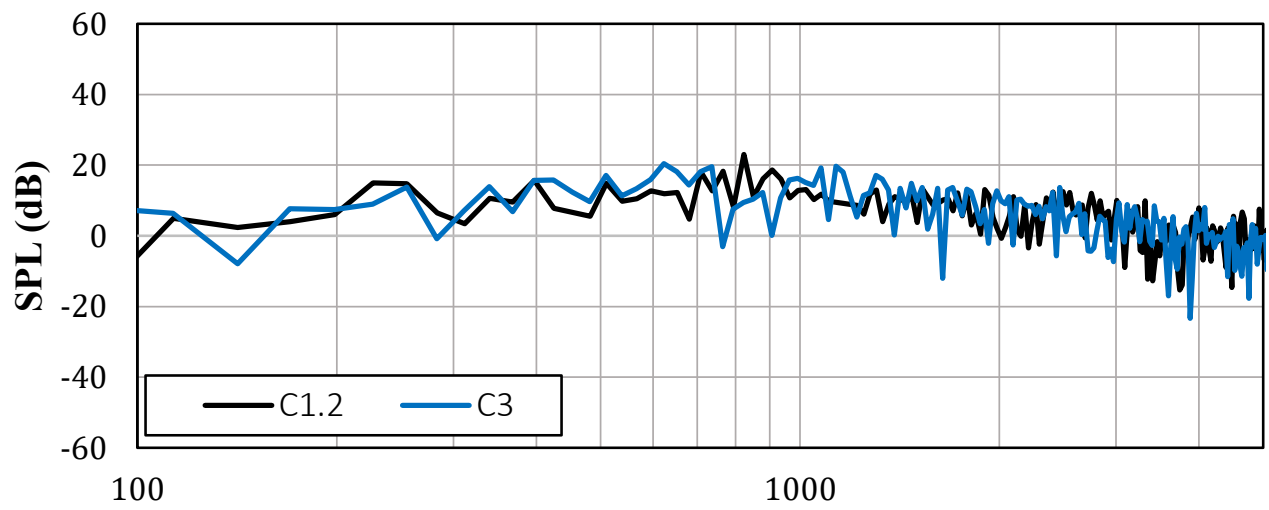

Frequency (Hz)

Figure 37: Frequency-domain noise signals of $\mathrm{C} 1.2$ and $\mathrm{C} 3$. 


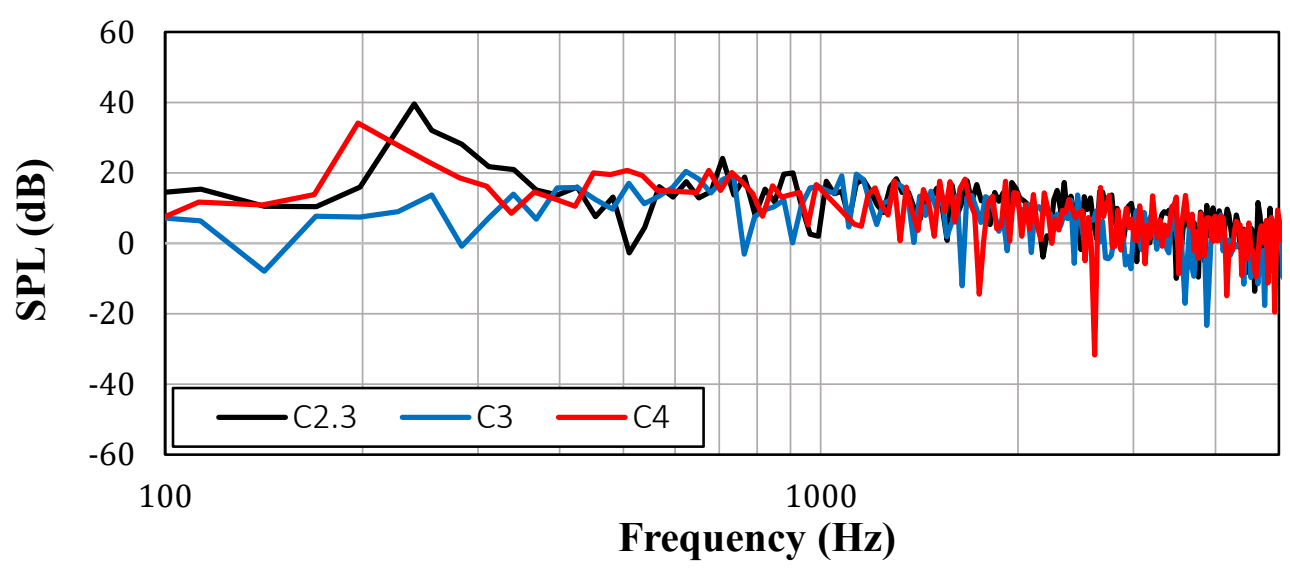

Figure 38: Frequency-domain pressure signals for $\mathrm{C} 2.3, \mathrm{C} 3$ and $\mathrm{C} 4$.

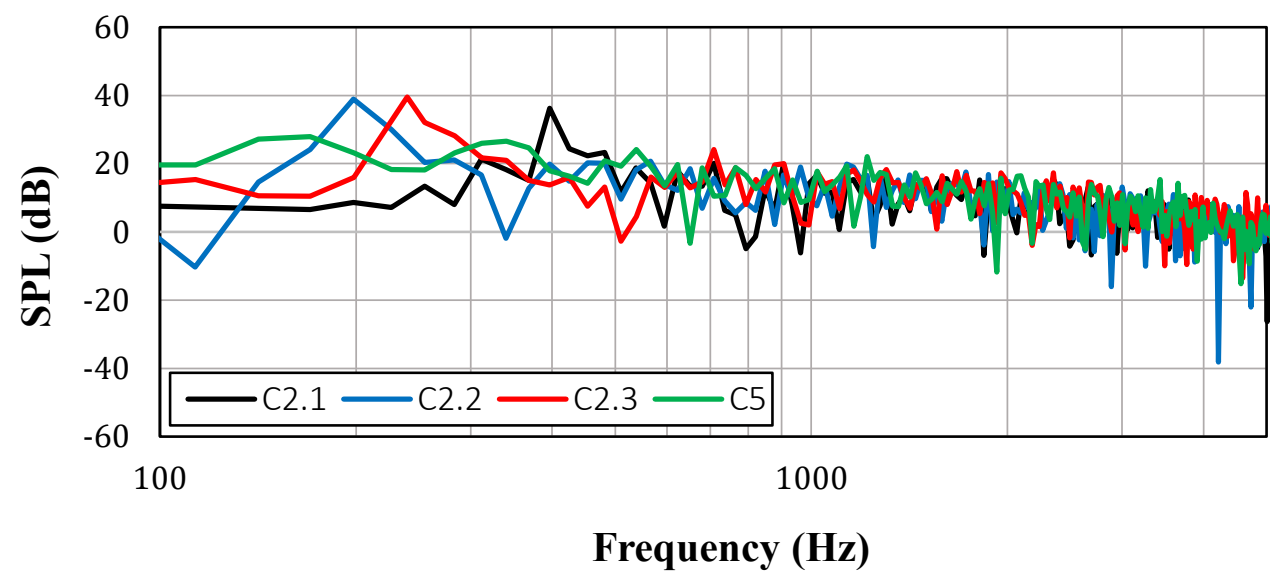

Figure 39: Frequency-domain pressure signals for $\mathrm{C} 2.1, \mathrm{C} 2.3$ and $\mathrm{C} 5$.

In summary, far-field noise is predicted for all the presented ELES cases using the FW-H aeroacoustic analogy. Clear periodic patterns are observed in the time-domain signals of C2.1, C2.2 and C2.3. Meanwhile, C4 and C5 show less defined periodic patterns. C1.2 and C3 yield irregular patterns. Discrete FFT is used to the obtained time-domain signals, in order to inspect the frequency-domain spectrum and better analyse the predicted sound. Tonal peaks are observed for $\mathrm{C} 2.1$, C2.2 and C2.3 at $397 \mathrm{~Hz}, 198 \mathrm{~Hz}$ and $240 \mathrm{~Hz}$ respectively. The peak amplitudes are equal for $\mathrm{C} 2.2$ and $\mathrm{C} 2.3$ but $3.3 \mathrm{~dB}$ lower in $\mathrm{C} 2.1$. A tonal peak is also observed in $\mathrm{C} 4$, but the amplitude of which is $5.5 \mathrm{~dB}$ smaller than in C2.3. Lastly, C5 yields an acoustic spectrum that is smooth and free of tonal peaks when compared to the cases of standard serrations, where the peak amplitude is reduced by $7 \mathrm{~dB}$ relative to $\mathrm{C} 2.2$ and $\mathrm{C} 2.3$, and $3.7 \mathrm{~dB}$ relative to $\mathrm{C} 2.1$. 


\subsection{Experimental Results}

The far-field aeroacoustic characteristics of TE finlets on a NACA0012 airfoil are experimentally investigated. Far-field noise obtained from the flow around a finned TE airfoil is measured and compared relative to a flat TE configuration for different angles of attack (AOAs). Measurements were obtained from a microphone placed $1.5 \mathrm{~m}$, midspan, directly above the airfoil TE. The topology of the tested design is similar to, and inspired by the computational case C3. Tests were performed in the Carleton University medium-speed wind tunnel aeroacoustic test section. Details of the test section can be found in Chapter 3 and references $[81,82]$. The acoustic data sampling frequency is set to $20 \mathrm{kHz}$ and the sampling period is 30 seconds, as explained in subsection 3.3.

In Figures 41 and 42, the obtained SPL levels are displayed for AOA $=0^{\circ}$, $5^{\circ},-5^{\circ}, 10^{\circ}$ and $-10^{\circ}$. For all the presented cases, the introduction of finlets did not alter the general shape of the acoustic spectra. The acoustic spectra kept the same broadband behavior, with slight differences in SPL amplitudes. The difference in amplitude between the 2 configurations is sensitive to the AOA, and is most pronounced in $\mathrm{AOA}= \pm 10^{\circ}$. Finlets did not affect the broadband behavior of the radiated noise and no tonal peaks are observed. The OASPL is calculated to better quantify the effect of finlets on the radiated noise. Table 8 shows the obtained noise reduction as a function of the airfoil AOAs, where

$$
\triangle \mathrm{OASPL}=O A S P L_{\text {finned } T E}-O A S P L_{\text {straight } T E}
$$

Figure 40 plots the values listed in Table 8 . The observed noise reduction increases as the AOA is increased, and the values are consistent for the same absolute angles $\left(\triangle \mathrm{OASPL}_{-5^{\circ}} \approx \Delta \mathrm{OASPL}_{5^{\circ}} \text { and } \Delta \mathrm{OASPL}_{-10^{\circ}} \approx \Delta \mathrm{OASPL}_{10^{\circ}}\right)^{\text {. Since noise reduction }}$ is a function of the AOA, and modifying the AOA changes the BL thickness, then the noise reduction efficiency of finlets is dependent on the BL thickness. The observed trend agrees with the findings of Gstrein et al. [33]. Figures 43 and 44 isolate the cases of finlets from those of a flat TE, and shows the effect of the AOA 
on the acoustic spectra for each case independently. The shape of the obtained signal remains almost constant as the AOA changes. Increasing the AOA induces a slight upwards shift in the acoustic spectra of both cases.

In summary, far-field measurements are presented for a finned TE NACA0012 airfoil. Finlets reduce the OASPL without altering the general trend of the acoustic spectra, when compared to a straight TE. The observed noise reduction increases as the AOA increases. For each TE configuration, increasing the AOA is shown to slightly shift the spectra upwards. The following subsection compares the experimental data with the computational results.

Table 8: Effect of AOA on $\triangle \mathrm{OASPL}$.

\begin{tabular}{cccccc}
\hline AOA $\left({ }^{\circ}\right)$ & $\mathbf{- 1 0}$ & $-\mathbf{5}$ & $\mathbf{0}$ & $\mathbf{5}$ & $\mathbf{1 0}$ \\
\hline$\Delta O A S P L(d B)$ & -0.761 & -0.681 & -0.337 & -0.696 & -0.782 \\
\hline
\end{tabular}

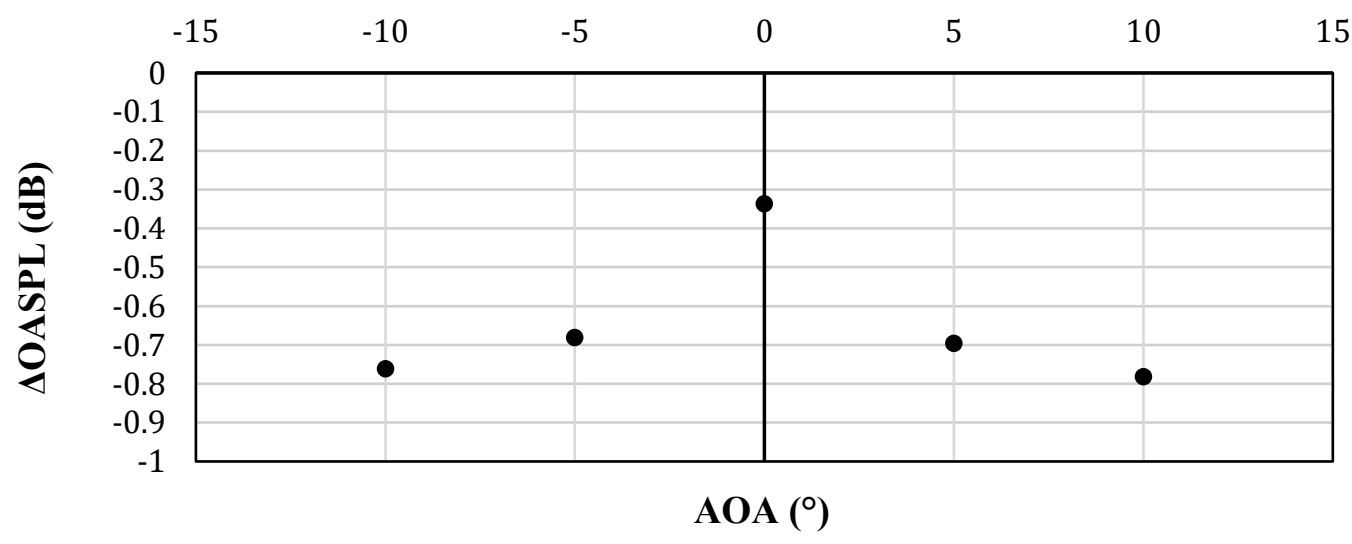

Figure 40: $\triangle \mathrm{OASPL}$ vs AOA. 

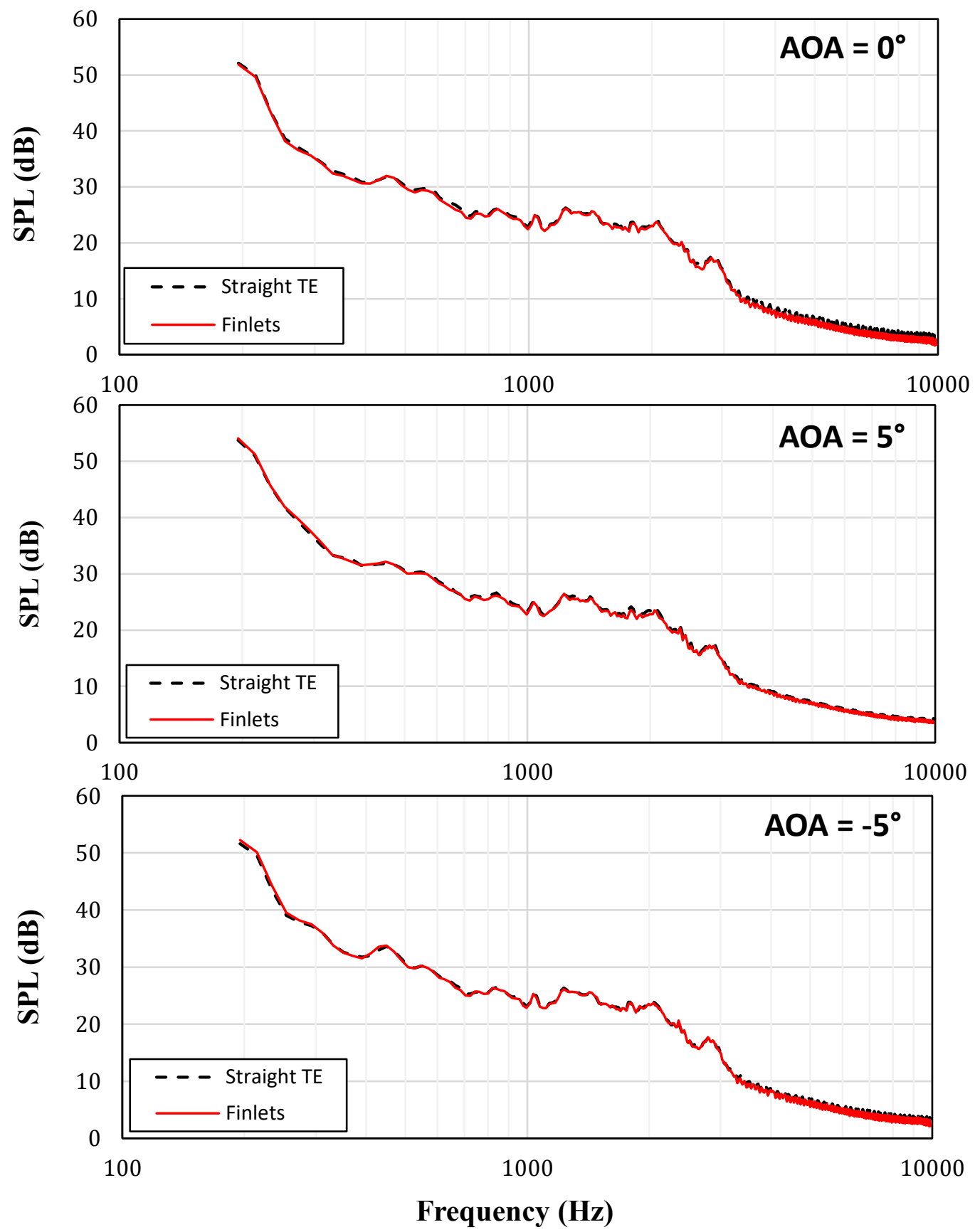

Figure 41: Experimentally obtained SPL, AOA $=0^{\circ}, 5^{\circ}$ and $-5^{\circ}$. 


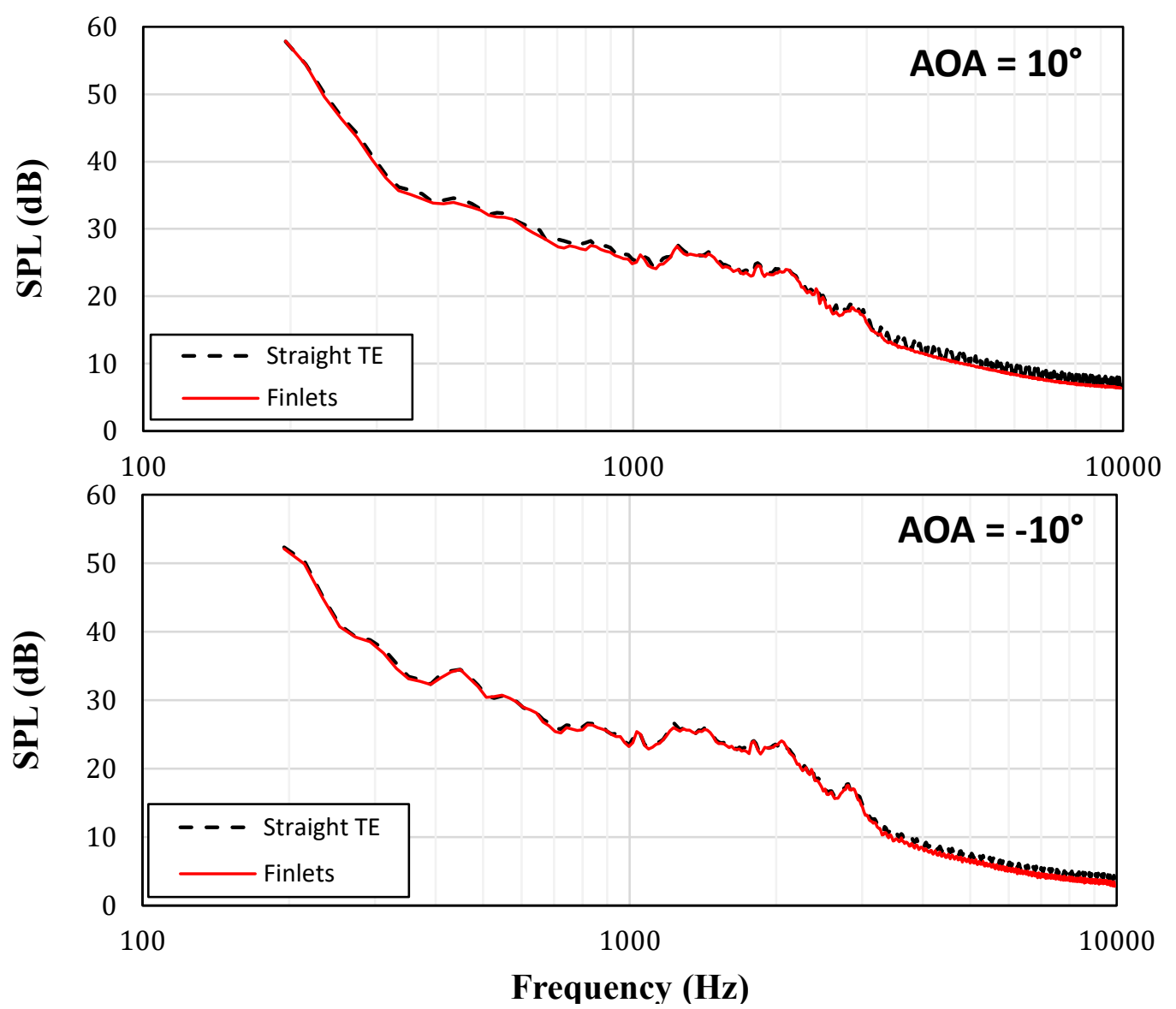

Figure 42: Experimentally obtained SPL, AOA $=10^{\circ}$ and $-10^{\circ}$.

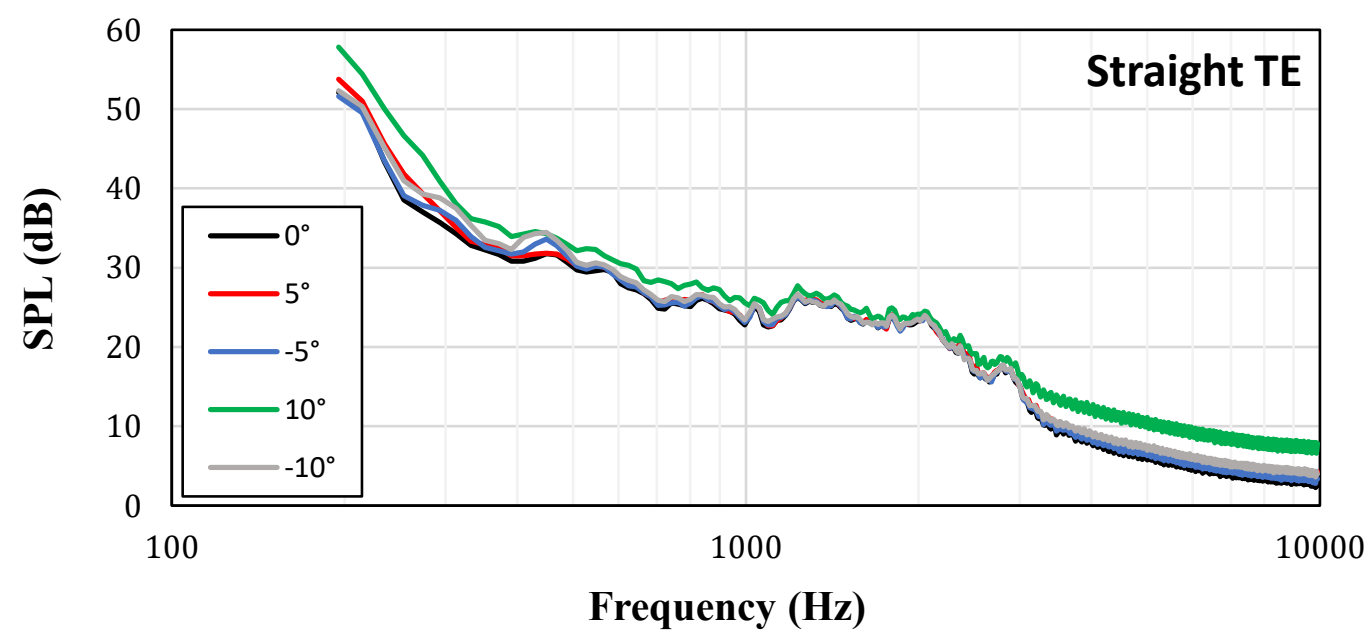

Figure 43: Experimentally obtained SPL, straight TE. 


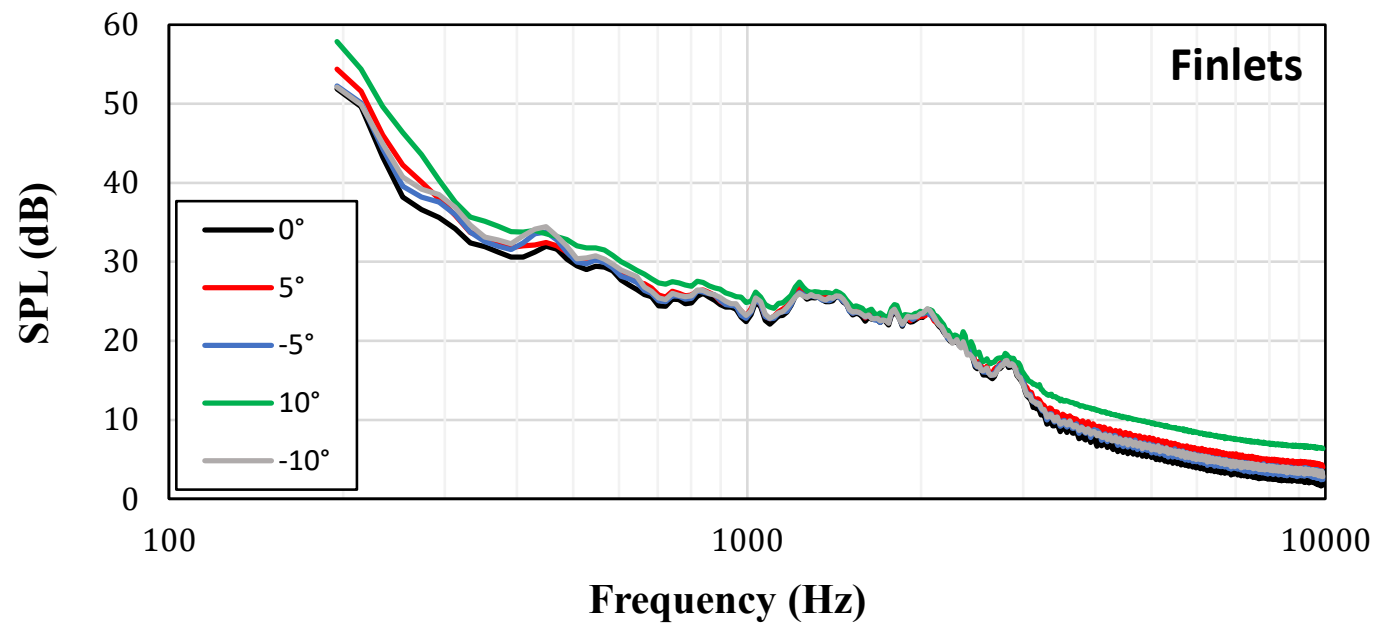

Figure 44: Experimentally obtained SPL, finlets.

\subsection{Comparison of Results}

In this subsection, a comparison of the available experimental and numerical results is presented. The numerical predictions of $\mathrm{C} 1.2$ are compared to the experimental results obtained from wind tunnel tests at $0^{\circ} \mathrm{AOA}$ (Figures 35 and 43). The overall sound levels in the experimental case are different from the computational case. It is believed that the cause for this difference is associated with the fact that the computational span is smaller than the experimental one $\left(\operatorname{span}_{\text {ELES }}=30 \mathrm{~mm}, \operatorname{span}_{\text {Experimental }}=510 \mathrm{~mm}\right)$. In addition, the noise source contribution of the airfoil LE is not accounted for in the used ELES domain configuration. Since the work is focused on TE noise, it is reasonable to neglect the noise radiated by other sections of the airfoil. The observed SPL trend and spectral fall-off are captured by the simulation and overall broadband noise behavior is obtained. Noise is generated as the hydrodynamic energy in the TBL is scattered into acoustic radiation. The turbulent fluctuations in the TBL can radiate sound directly, but it is their close proximity to the airfoil surface that amplifies the sound generated in subsonic flows [37]. This sound amplification is caused by to the 
unsteady surface pressure fluctuations on the airfoil surface, which lead to more efficient sound radiation when compared to free turbulence.

Figure 45 shows experimental SPL results obtained by B. Al Tlua $[81,82]$ for a serrated TE NACA0012 airfoil having $2 \mathrm{~h}=60 \mathrm{~mm}, \lambda=10 \mathrm{~mm}$ and $\epsilon=16.6 \mathrm{~mm}$. The experiments were conducted in the same wind tunnel facility and using the same variable-TE airfoil described in Chapter 3. The tested airfoil is similar to that of simulation C2.3. Figure 35 shows the numerically predicted SPL levels for $\mathrm{C} 2.3$. The numerical simulations and the wind tunnel experiments predict comparable acoustic behavior. The numerically simulated and experimentally obtained SPL levels follow similar acoustic spectra shapes, but with different amplitudes. This is caused by limited computational domain span, as discussed in the case of a flat TE. Narrowband tonal peaks are predicted in both numerical and experimental far-field spectra. Both numerical and experimental results show tonal peaks at $240 \mathrm{~Hz}$ and $290 \mathrm{~Hz}$, respectively, which is caused by vortex shedding associated with the bluntness of the serration roots. The difference in the predicted tonal frequencies can be attributed to the subtle differences that exist between the numerical and experimental geometric models and inflow conditions, such as turbulence, the limited size of the LES domain, and the intrinsic errors of the approximations used to decouple noise generation from its propagation. The observed difference between numerical and experimental tonal peak frequencies is consistent with the results of Kim et al. [64], who used a similar ELES domain configuration for predicting aerodynamic noise generation by vortical flows in the vicinity of a blunt TE and compared the output to wind tunnel experiments. Experimental results confirm that sawtooth serrations reduce the generated broadband noise at the expense of adding a narrow-band tonal peak. The broadband reduction was not captured in the numerical simulations. Increasing the sensitivity of the simulation would require a finer mesh and longer simulation time, which would render the simulations unfeasible. For the broadband reduction to be captured without increasing the simulation run-time, more powerful computer clusters are required to allow for a larger LES domain and a higher frequency resolution (longer sampling period). The obtained accuracy is found to be 
satisfactory for the purposes discussed in this study, when compared to the corresponding reduction in computing effort. ELES successfully predicted the narrowband tonal noise component at a relatively low computational cost, and was used to assess the effect of different serration parameters on the tonal peak and frequency. Table 9 summarizes the geometric parameters of the standard serration cases and the corresponding tonal peaks.

Altogether, the presence of serrations mitigates the sudden interaction between the TBLs on the upper and lower sides of the airfoil, thus allowing for the progressive mixing previously discussed, and affecting the radiated broadband sound. C2.2 and C2.3 experience the highest values in terms of $P_{r m s}$ near the serration roots, amplitudes of lift-coefficient variation, amplitudes of vortex shedding and finally, far-field tonal peak amplitudes. This is an indication that longer serrations, and higher values of $\epsilon$, are responsible for the strength of the radiated tonal noise. Furthermore, the frequency at which the tonal peak occurs can be controlled for the same $2 \mathrm{~h}$ and $\epsilon$ by modifying the wavelength, $\lambda$. Larger values of $\lambda$ lead to higher tonal peak frequencies, and larger values of $\epsilon$ lead to lower tonal peak frequencies accompanied by higher amplitudes.

Table 9: Summary of standard serration characteristics and corresponding tonal peak frequencies.

\begin{tabular}{lcccc}
\hline Case & $\boldsymbol{2 h}(\boldsymbol{m m})$ & $\boldsymbol{\lambda}(\boldsymbol{m m})$ & $\boldsymbol{\epsilon}(\boldsymbol{m m})$ & Frequency $(\mathbf{H z})$ \\
\hline $\mathbf{C 2 . 1}$ & 30 & 10 & 7.4 & 397 \\
\hline $\mathbf{C 2 . 2}$ & 60 & 7.5 & 16.3 & 198 \\
\hline $\mathbf{C 2 . 3}$ & 60 & 10 & 16.3 & 240 \\
\hline
\end{tabular}




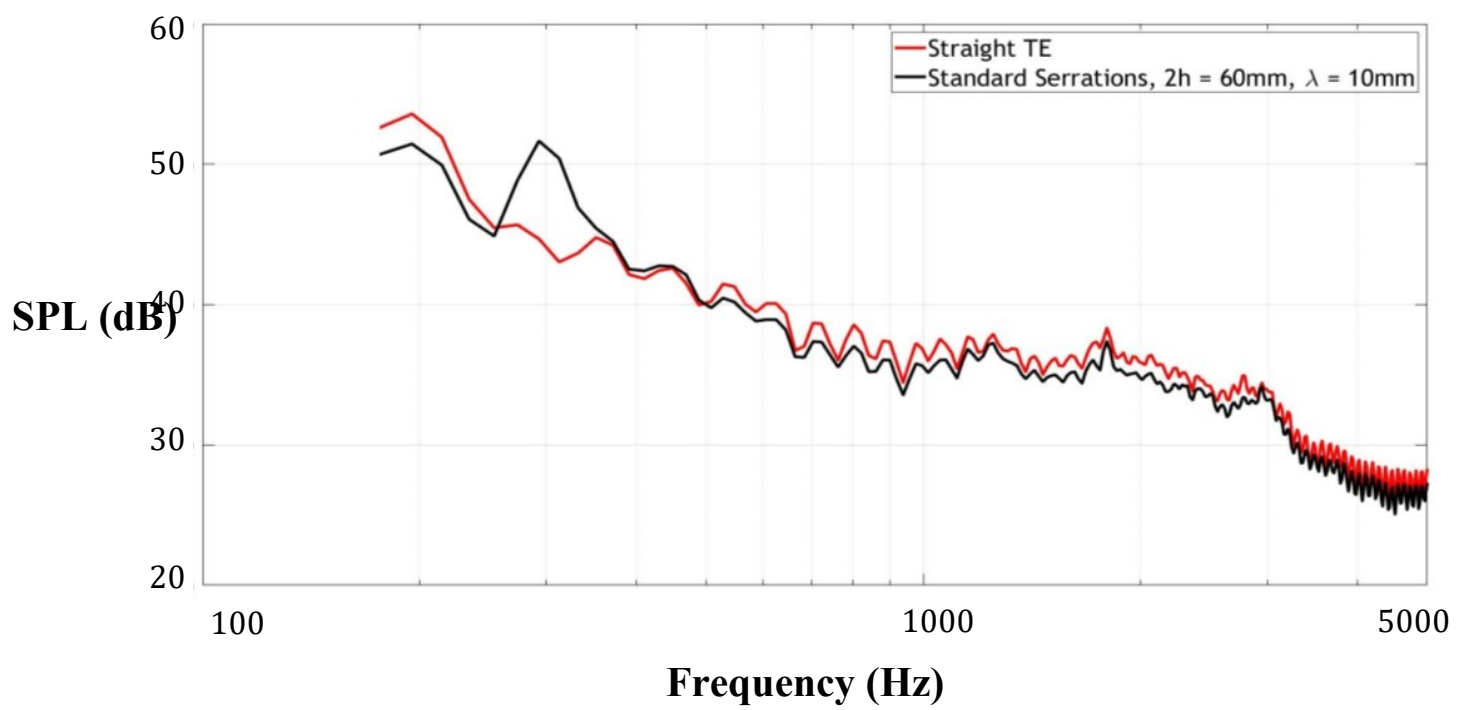

Figure 45: Experimentally obtained SPL by B. Al Tlua [81,82].

A NACA0012 airfoil with finlets attached to the TE was numerically simulated using ELES and experimentally tested in the Carleton University medium speed wind tunnel. Figure 37 shows the numerical predictions at $0^{\circ} \mathrm{AOA}$ while Figure 44 shows the experimental results at $\mathrm{AOA}=-10^{\circ},-5^{\circ}, 0^{\circ}, 5^{\circ}$ and $10^{\circ}$. Agreement between the numerical are experimental results is achieved at $\mathrm{AOA}=0^{\circ}$, in terms of acoustic spectral characteristics and general trend. A broadband reduction of $0.448 \mathrm{~dB}$ was predicted by the numerical simulation, for frequencies between $1000 \mathrm{~Hz}$ and $5000 \mathrm{~Hz}$. Experimental results show an OASPL reduction of $0.337 \mathrm{~dB}$ between 200 and $10,000 \mathrm{~Hz}$. Wind tunnel results show that the obtained noise reduction varies with the airfoil AOA. Since changing the AOA changes the thickness of the $\mathrm{BL}$, results agree with the findings of Gstrein et al. [33], who suggested that noise reduction in finlets is dependent on the BL thickness. Finlets lead to the break-up of large coherent structures by forcing them between the introduced channels. The size of the channeled turbulent coherent structures can be controlled by changing the spacing of the finlets. Finer spacing will lead to a more pronounced reduction in the high frequency noise, while coarser spacing will have that effect on low frequency noise. Finlets serve to decorrelate the BL in the spanwise direction and convect the energetic eddies away from the airfoil surface, decreasing the velocity of the fluid particles that are in proximity of the sharp TE, 
and subsequently reducing the efficiency of acoustic scattering [34,96]. It's important to note that the noise scattered from a half plane is proportional in its intensity to the fifth power of flow velocity [96]. It is then reasonable to conclude that the degree to which turbulent structures are cut, deformed and decorrelated is the main driver in this noise reduction mechanism [29]. The noise reduction mechanisms seen in finlets are fundamentally different from the ones observed in the cases of standard serrations. 


\section{Chapter 6}

\section{Conclusions and Recommendations}

This chapter presents the conclusions from the current study. ELES and experimental tests were performed with the purpose of investigating the aerodynamic and aeroacoustic performance of different bio-inspired TE configurations. A summary of all the results is presented in subsection 6.1, and conclusions in subsection 6.2. Lastly, recommendations for future improvements is provided in subsection 6.3 .

\subsection{Summary of Results}

This thesis outlines the numerical simulations and wind tunnel tests conducted to investigate the far-field noise radiated by different bio-inspired noisereducing TE designs, and identify the flow mechanisms responsible for generating noise. A numerical framework was developed, where ELES is coupled with the FW-H aeroacoustic analogy, to avoid the need for traditionally used, prohibitively expensive LES models, while still obtaining accurate results. Results from two preliminary ELES simulations ( $\mathrm{C} 1.1$ and $\mathrm{C} 1.2)$ were presented, in which the applicability of ELES in tandem with the FW-H analogy was assessed. Results were compared against published experimental and numerical results, and mesh convergence tests were performed. The better configuration (C1.2) was carried over, along with the best-suited mesh, for the latter simulations (C2.1, C2.2, C2.3, $\mathrm{C} 3, \mathrm{C} 4$ and C5). Time-averaged $P_{r m s}$ surface-contours were generated to locate the regions of maximum pressure fluctuations in most case. The instantaneous TKE distributions at different streamwise locations of interest were presented and used 
to understand the effect of the different designs on the development of the TBL. Lift-coefficient history plots were obtained to assess the effect of each design on the fluctuating lift forces acting on the airfoil. Lift-coefficient history plots also serve to demonstrate the obtained statistical convergence of the simulations. The effect of each TE design on the airfoil wake was explored through streamwise contours of vorticity magnitude, and the underlying 3-dimensional turbulent structures were displayed in terms of Q-Criterion iso-surfaces. The streamwise vorticity, $\omega_{x}$, was used to investigate the quality of mixing at the TE of each design, and show the size of the coherent structures. Using the FW-H aeroacoustic model, pressure fluctuations were propagated to a far-field receiver placed outside of the CFD domain, and the frequency-domain signals were obtained by applying FFT on those time-pressure noise signals. Frequency-domain SPL spectra were presented and used to quantify the radiated noise and investigate the occurrence of any narrow-band tonal peaks.

\subsection{Conclusion}

The initial phase of this research focused on the development of highfidelity, computationally affordable simulations for aeroacoustic noise prediction. Traditional models, such as LES and DNS, are notorious for their extreme computational power and time requirements, which makes them unfeasible in most industrial projects. The need for reliable, relatively short-response-time noise prediction methods is addressed. ELES was chosen, where the flow domain was split into RANS and LES regions. ELES allowed for the relaxation of the otherwise very strict LES mesh requirements in the RANS region, leading to a significant reduction in the overall number of mesh elements and notably reducing the runtime of the simulations. Mesh convergence tests were carried out to establish trust in the numerical predictions, and ELES results were compared against full LES and experimental results, to ensure the natural growth and development of the TBL. The 
developed framework was then utilized to investigate the impact of different bioinspired TE designs on noise radiation, as well as advance the understanding of the underlying flow physics. Simulations were carried out for a NACA0012 airfoil at a freestream flow velocity $u_{\infty}=24 \mathrm{~m} / \mathrm{s}$, AOA $=0$ and $\mathrm{Re}_{\mathrm{c}}$ of approximately 500,000 .

Experimental wind tunnel testing was conducted at similar flow conditions, in the Carleton University Medium Speed Wind Tunnel, for a straight TE airfoil and a finned TE airfoil to assess the effect of AOA of noise radiation. The case of a straight TE NACA0012 airfoil (C1.2) was numerically and experimentally investigated. Noise is generated as the hydrodynamic energy contained in the TBL is scattered as acoustic radiation. Although the turbulent pressure fluctuations in TBL can directly radiate sound, it is their proximity to the airfoil surface and the existence of a singularity at the airfoil TE that amplify this noise and efficiently scatter it. At the TE, two independent TBLs come together and collide for the first time, giving rise to violent pressure fluctuations.

Three different standard-serration configurations (C2.1, C2.2 and C2.3) having different geometric parameters were simulated and results compared. Standard TE serrations were initially inspired by the fringe found in the TE of owl wings. Longer serrations were shown to promote turbulence and improve mixing. Sinusoidal vorticity magnitude and Q-Criterion iso-surface distributions suggested that alternating vortices were being shed in the airfoil wake. The vortex shedding frequencies for C2.1, C2.2 and C2.3 were $388 \mathrm{~Hz}, 218 \mathrm{~Hz}$ and $248 \mathrm{~Hz}$, respectively. The bluntness of the standard serration roots, $\epsilon$, is responsible for the observed vortex shedding. Wake vorticity in all cases was dissipated faster than in C1.2. Narrow-band tonal peaks were observed in the far-field noise spectra at $397 \mathrm{~Hz}$, $198 \mathrm{~Hz}$ and $240 \mathrm{~Hz}$ for C2.1, C2.2 and C2.3, respectively. The tonal peak amplitudes were equal for $\mathrm{C} 2.2$ and $\mathrm{C} 2.3$, which share the same $2 \mathrm{~h}=60 \mathrm{~mm}$ and $\epsilon=16.3 \mathrm{~mm}$. The peak amplitude was lower in $\mathrm{C} 2.1$ by $3.3 \mathrm{~dB}$, suggesting that the tonal noise is louder for cases having longer serrations and increased root bluntness. The numerical predictions were compared to the experimental findings of $\mathrm{B}$. Al Tlua $[81,82]$ for an airfoil having geometric characteristics similar to $\mathrm{C} 2.3$, where 
a tonal peak was observed at $290 \mathrm{~Hz}$. By simulating C2.3 twice using two different computational meshes, it was shown that the used mesh has no effect on the predicted far-field tonal peaks, thus demonstrating mesh convergence. The observed tonal peaks are justified by the phenomenon of vortex shedding, and the frequencies of occurrence are correlated. The presence of standard serrations was shown to mitigate the sudden interaction between the TBLs on the upper and lower sides of the airfoil, thus allowing for progressive mixing and attenuating the radiated broadband noise, at the expense of a narrow-band tonal peak. Longer serrations, and higher values of $\epsilon$ are responsible for the strength of the shed vortices and the intensity of the radiated tonal noise. The frequency at which the tonal peaks occur can be controlled for the same $2 \mathrm{~h}$ and $\epsilon$ by modifying the wavelength, $\lambda$. Larger values of $\lambda$ for the same $2 \mathrm{~h}$ and $\epsilon$ lead to higher tonal peak frequencies, and larger values of $\epsilon$ for the same $2 \mathrm{~h}$ and $\lambda$ lead to lower tonal peak frequencies accompanied by higher peak amplitudes.

Finlets, which are a practical means of applying a feature similar to the downy upper coat of owl wings on solid airfoils, were investigated in case C3. The TBL was decorrelated in the spanwise direction and the size of the counter-rotating eddies near the TE was reduced. The fluctuating lift forces the airfoil itself is subject to with a finned TE (C3) are almost the same as for a clean one (C1.2), less than in any of the cases of standard serrations. C3 predicted a broadband SPL curve similar to that of $\mathrm{C} 1.2$, in terms of general trend, and no tonal peaks were present. An OASPL reduction of $0.488 \mathrm{~dB}$ was predicted in the frequency range $1000 \mathrm{~Hz}$ to $5000 \mathrm{~Hz}$. Wind tunnel testing showed the effect of the airfoil AOA on obtained sound reduction. For $\mathrm{AOA}=-10^{\circ},-5^{\circ}, 0^{\circ}, 5^{\circ}$ and $10^{\circ}$, OASPL reductions of 0.761 $\mathrm{dB}, 0.681 \mathrm{~dB}, 0.337 \mathrm{~dB}, 0.696 \mathrm{~dB}$ and $0.782 \mathrm{~dB}$ were obtained, respectively. $\triangle$ OASPL between a flat TE and a finned TE was shown to be sensitive to the AOA, and is largest at $\mathrm{AOA}= \pm 10^{\circ}$. The experimental results confirmed the dependence of finlet behavior on the thickness of the BL. The obtained reductions were also consistent for the same absolute AOAs. Qualitative agreement between the numerical are experimental results is achieved, in terms of spectral characteristics and general trend. Finlets deform and decorrelate the large coherent structures of 
the TBL before they reach the airfoil TE, decreasing the velocity of the fluid particles around the sharp TE, thus reducing the efficiency of acoustic scattering and provoking a broadband noise reduction. The size of the turbulent coherent structures can be manipulated by changing the finlet spacing, S, such that spacings finer spacing would lead to smaller turbulent structures that radiate a higher frequency noise, and coarser spacings would lead to larger structures radiating lower frequency noise. The noise reduction mechanisms observed in finlets are fundamentally different from those found in standard serrations

In case $\mathrm{C} 4$, a new TE design, termed "finned serrations", was presented to look into the possibility of superimposing the different noise reduction mechanisms finlets plus serrations. Finned serrations were shown to successfully channel the flow and decorrelate the TBL in the spanwise direction, as well as improve mixing across the airfoil surface, successfully combining the aerodynamic flow features of having finlets and serrations. The size of turbulent coherent structures near the TE is decreased and a phase shift is observed between the different flow channels, which can be leveraged in future designs to promote destructive acoustic interference. Vortices were shed from the blunt serration roots leading to a tonal peak in the radiated far-field noise. The tonal amplitude was reduced by $5.5 \mathrm{~dB}$ in comparison to standard serrations with the same serration amplitude (C2.2 and $\mathrm{C} 2.3$ ), and the corresponding frequency was less. Tonal attenuation is explained by the weakening of vortex shedding, which is attributed to the flow channeling and decorrelation. By modifying the spacing and thickness of finlets, relative to the amplitude and wavelength of serrations, tonal noise can be reduced and its frequency can be manipulated. Results suggest that coupling different noisesuppressing flow mechanisms is a promising approach to design new, quieter airfoils. More research is required to guarantee proper coupling of the parameters of finlets and serrations, and induce a pronounced broadband noise reduction.

A design similar to the slanted-root serrations proposed by Chen et al. [28] was simulated in $\mathrm{C} 5$ to investigate the effect of slanting standard serration roots on vortex shedding and tonal noise radiation. Alternating slanting ratios of 0.5 and 2 were used. By slanting serration roots, symmetry of the flow around the $z-x$ plane 
was broken, and the periodic cycle of vortex shedding was destabilized, aiding in the dissipation of the alternating low-pressure zones being shed. Slanted-root serrations also promoted streamwise vorticity, $\omega_{x}$, and improved mixing across the airfoil as standard serrations would. Some similarities were observed between finlets (C3) and slanted-root serrations (C5). The first similarity was the flow channeling effect and the second was the reduction of flow velocity near the TE, which in turn weakens acoustic scattering. The tonal peaks present in cases C2.1, $\mathrm{C} 2.2$ and $\mathrm{C} 2.3$ were reduced by $3.7 \mathrm{~dB}, 7 \mathrm{~dB}$ and $7 \mathrm{~dB}$, respectively, in C5. Slanting the roots of standard serrations results in an improvement in terms of tone attenuation, but the serration amplitude might need to be increased to account for the effective mixing length, which is reduced when the roots are slanted. Results demonstrate the successful suppression of the narrowband tonal peak observed in the case of standard serrations using slanted-root serrations.

Comparisons were made between computational results and experimental measurements and good agreement was achieved. ELES was shown to provide accurate predictions of pressure statistics, vorticity, and wake characteristics. In combination with the FW-H analogy, ELES successfully predicted narrowband peaks in the radiated far-field noise of $\mathrm{C} 2.1, \mathrm{C} 2.2, \mathrm{C} 2.3$ and $\mathrm{C} 4$, as well as the suppression of the tonal peak in $\mathrm{C} 5$. Broadband noise reduction was captured in $\mathrm{C} 3$. The results of this investigation illustrate how ELES can be used as an alternative in the preliminary design phase to the currently used more computationally demanding full LES, or DNS approaches. It's worth noting that the simulations presented by Romani et al. [67], for example, were run on an Intel Xeon E5-2690 $2.90 \mathrm{GHz}$ platform of 360 cores, and the CPU runtime per flow passage for different mesh resolutions was up to 2100 hours and 15600 hours, while for all the presented ELES, the run-time was on average 200 hours/flow passage on an Intel Xeon L5410 $2.33 \mathrm{GHz}$ platform of 60 cores. Future work aims to utilize the aforementioned method and results for the development of new noise-suppressing TE designs. 


\subsection{Future Recommendations}

The following recommendations and future work have been identified for the numerical and experimental setups:

1. In ELES, synthetic turbulence was injected at the RANS/LES interface using the Vortex Method. The size of the injection region was kept larger than the BL thickness, since the injected turbulence is a function of the mean flow, and the mean flow is not as turbulent outside the TBL as it is inside. While not expected to be a major source of error, this effect can be investigated and optimum turbulence injection regions can be specified in future simulations.

2. While tonal peaks were successfully predicted using ELES, the configuration and mesh were not sensitive enough to capture the broadband reduction in all the presented cases. Further improvements to the computational mesh can be considered, in which the surface elements can be progressively refined to increase the overall sensitivity to pressure fluctuations, until broadband reduction is captured. It's also crucial to be careful not to over-refine the mesh elements as that could cause noise and contaminate the high-frequency noise predictions. The main purpose of ELES is to overcome the impractical cost of DNS and full LES, so it's important not to lose sight of the objective when trying to improve the sensitivity. The obtained predictions constitute a trade-off between accuracy and computational cost, where the cost was significantly truncated and the results were kept sufficiently accurate.

3. To obtain the current results, simulations would run on a 60-core computer cluster at Carleton University for an average of 672 hours, split between flow initialization and turbulent and acoustic data sampling. The total simulated flow time in those 672 hours is only 0.101 seconds, while experimental testing had a sampling period of 30 seconds. More accurate 
numerical predictions can be obtained without increasing the simulation run-time at the expense of acquiring more powerful computers. Then, the total simulated flow-time can be increased for the same amount of computer run-time, leading to a longer sampling period and higher accuracy.

4. The far-field experimental measurements could not be carried out at angles larger than $\pm 10^{\circ}$ due to the size limitations of the test section. Ideally, it would have been interesting to investigate the effect of finlets on noise reduction near stall, and their effect on the stall angle itself.

5. Compliant surfaces could be used to improve the behavior of standard serrations if they are strategically added near the regions of maximum pressure fluctuations. Their flexibility is expected provide a bypass mechanism for turbulence energy dissipation, which would weaken the shed vortices and improve the overall noise characteristics.

6. Based on the results obtained for finned serrations (C4), it is recommended to test the effect of changing the finlet spacing while keeping the serration parameters constant. Since TBL decorrelation aids in the attenuation of tonal peaks, unequal finlet spacing could also be a promising design to pursue.

7. The slanting ratio in simulation $\mathrm{C} 5$ was chosen to alternate between 0.5 and 2. Future work could look into different slanting ratios and assess their effect on vortex shedding and noise radiation.

8. Future experiments and simulations could do a directivity study by changing the receiver location and investigate the effect of the different TEs on noise at different polar angles and locations in the far-field.

9. Conducting an experimental study to further investigate the sensitivity of the results to specific TE geometric parameters, such as the serration amplitude in TE serrations and the spacing between finlets in TE finlets. 


\section{Bibliography}

[1] Ortega, Catherine P. "Chapter 2: Effects of noise pollution on birds: A brief review of our knowledge." Ornithological monographs 74.1 (2012): 6-22.

[2] Bronzaft, Arline L. "Noise pollution: A hazard to physical and mental wellbeing." Handbook of environmental psychology (2002): 499-510.

[3] Secretariat, I. C. A. O. "Aviation Outlook Overview." BAN Ki-moon.

[4] ICAO Secretariat, Reducing Aircraft Noise - Overview, ICAO Environmental Report, Chapter 2 (2016).

[5] Brooks, Thomas F., D. Stuart Pope, and Michael A. Marcolini. Airfoil self-noise and prediction. Vol. 1218. Washington, DC: National Aeronautics and Space Administration, Office of Management, Scientific and Technical Information Division, 1989.

[6] Naterer, Greg, Ofelia Jianu, and Marc Rosen. "Noise Polution Prevention in Wind Turbines: Status and Recent Advances." (2011).

[7] Graham, R. R. "The silent flight of owls." The Aeronautical Journal 38.286 (1934): 837-843.

[8] Williams, J.E. Ffowcs, and David L. Hawkings. "Sound generation by turbulence and surfaces in arbitrary motion." Philosophical Transactions for the Royal Society of London. Series A, Mathematical and Physical Sciences (1969): 321-342.

[9] Hertel, Heinrich. "Struktur, Form, Bewegung." (1963).

[10] Gruschka, H. D., I. U. Borchers, and J. G. Coble. "Aerodynamic noise produced by a gliding owl." Nature 233.5319 (1971): 409-411.

[11] Kroeger, Richard A., Heinz D. Grushka, and Tibor C. Helvey. Low speed aerodynamics for ultra-quiet flight. Tennessee Univ Space Inst Tullahoma, 1972. 
[12] Lilley, Geoffrey. "A study of the silent flight of the owl." 4th AIAA/CEAS aeroacoustics conference. 1998.

[13] Bachmann, Thomas, et al. "Morphometric characterisation of wing feathers of the barn owl Tyto alba pratincola and the pigeon Columba livia." Frontiers in zoology 4.1 (2007): 1-15.

[14] Bachmann, Thomas, and Hermann Wagner. "The three-dimensional shape of serrations at barn owl wings: towards a typical natural serration as a role model for biomimetic applications." Journal of anatomy 219.2 (2011): 192-202.

[15] Wagner, Hermann, et al. "Features of owl wings that promote silent flight." Interface focus 7.1 (2017): 20160078.

[16] Arndt, R. "Effect of leading edge serrations on noise radiation from a model rotor." Society of Naval Architects and Marine Engineers, and US Navy, Advanced Marine Vehicles Meeting. 1972.

[17] Hersh, Alan S., Paul T. Soderman, and Richard E. Hayden. "Investigation of acoustic effects of leading-edge serrations on airfoils." Journal of Aircraft 11.4 (1974): 197-202.

[18] Klän, Stephan, Michael Klaas, and Wolfgang Schröder. "The influence of leading edge serrations on the flow field of an artificial owl wing." 28th AIAA Applied Aerodynamics Conference. 2010.

[19] Schwind, R. G., and H. J. Allen. "The effects of leading-edge serrations on reducing flow unsteadiness about airfoils, an experimental and analytical investigation." (1973).

[20] Narayanan, S., et al. "Airfoil noise reductions through leading edge serrations." Physics of Fluids 27.2 (2015): 025109.

[21] Herr, Michaela, and Werner Dobrzynski. "Experimental Investigations in Low-Noise Trailing Edge Design." AIAA journal 43.6 (2005): 1167-1175.

[22] Gharali, Kobra, Nicholas Tam, and David A. Johnson. "A PIV load and flow structure study of a serrated dynamic airfoil." Lisbon 17th Int. Symp. On Applications of Laser Techniques to Fluid Mechanics, Lisbon, Portugal. 2014.

[23] Liang, G., et al. "The study of owl's silent flight and noise reduction on fan vane with bionic structure." Advances in Natural Science 3.2 (2010): 192-198. 
[24] Gruber, Mathieu, Phillip Joseph, and Mahdi Azarpeyvand. "An experimental investigation of novel trailing edge geometries on airfoil trailing edge noise reduction." 19th AIAA/CEAS aeroacoustics conference. 2013.

[25] Chong, Tze Pei, et al. "Self-noise produced by an airfoil with nonflat plate trailing-edge serrations." AIAA journal 51.11 (2013): 2665-2677.

[26] Gruber, Mathieu, Phillip Joseph, and Tze Chong. "On the mechanisms of serrated airfoil trailing edge noise reduction." 17th AIAA/CEAS aeroacoustics conference (32nd AIAA aeroacoustics conference). 2011.

[27] Howe, Michael S. "Noise produced by a sawtooth trailing edge." The Journal of the Acoustical society of America 90.1 (1991): 482-487.

[28] Chen, Eryun, et al. "Experimental investigation on noise emissions of an airfoil with non-flat plate trailing edge serrations." Journal of Mechanical Science and Technology 33.7 (2019): 3069-3074.

[29] Clark, Ian A., et al. "Bioinspired trailing-edge noise control." AIAA Journal 55.3 (2017): 740-754.

[30] Afshari, Abbas, et al. "Effects of streamwise surface treatments on trailing edge noise reduction." 23rd AIAA/CEAS Aeroacoustics Conference. 2017.

[31] Shi, Yuejun, and Seongkyu Lee. "Numerical study of 3-D finlets using Reynolds-averaged Navier-Stokes computational fluid dynamics for trailing edge noise reduction." International Journal of Aeroacoustics 19.1-2 (2020): 95-118.

[32] Afshari, Abbas, et al. "Trailing-edge flow manipulation using streamwise finlets." Journal of Fluid Mechanics 870 (2019): 617-650.

[33] Gstrein, Felix, Nick Zang, and Mahdi Azarpeyvand. "Application of Finlets for Trailing Edge Noise Reduction of a NACA 0012 Airfoil." AIAA AVIATION 2020 FORUM. 2020.

[34] Bodling, Andrew, and Anupam Sharma. "Numerical investigation of lownoise airfoils inspired by the down coat of owls." Bioinspiration \& biomimetics 14.1 (2018): 016013. 
[35] Lighthill, Michael James. "On sound generated aerodynamically I. General theory." Proceedings of the Royal Society of London. Series A. Mathematical and Physical Sciences 211.1107 (1952): 564-587.

[36] Lighthill, Michael James. "On sound generated aerodynamically II. Turbulence as a source of sound." Proceedings of the Royal Society of London. Series A. Mathematical and Physical Sciences 222.1148 (1954): 1-32.

[37] Curle, N. "The influence of solid boundaries upon aerodynamic sound." Proceedings of the Royal Society of London. Series A. Mathematical and Physical Sciences 231.1187 (1955): 505-514.

[38] Chase, David M. "Sound radiated by turbulent flow off a rigid half-plane as obtained from a wavevector spectrum of hydrodynamic pressure." The Journal of the Acoustical Society of America 52.3B (1972): 1011-1023.

[39] Chase, David M. "Noise radiated from an edge in turbulent flow." AIAA journal 13.8 (1975): 1041-1047.

[40] Chandiramani, K. L. "Diffraction of evanescent waves, with applications to aerodynamically scattered sound and radiation from unbaffled plates." The Journal of the Acoustical Society of America 55.1 (1974): 19-29.

[41] Amiet, Roy K. "Acoustic radiation from an airfoil in a turbulent stream." Journal of Sound and vibration 41.4 (1975): 407-420.

[42] Howe, Michael S. "A review of the theory of trailing edge noise." Journal of sound and vibration 61.3 (1978): 437-465.

[43] Howe, Michael S. "Aerodynamic noise of a serrated trailing edge." Journal of Fluids and Structures 5.1 (1991): 33-45.

[44] Roger, Michel, and Stéphane Moreau. "Back-scattering correction and further extensions of Amiet's trailing-edge noise model. Part 1: theory." Journal of Sound and vibration 286.3 (2005): 477-506.

[45] Mayer, Yannick D., et al. "A semi-analytical noise prediction model for airfoils with serrated trailing edges." Renewable Energy 143 (2019): 679-691.

[46] Lyu, Benshuai, Mahdi Azarpeyvand, and S. Sinayoko. "Prediction of noise from serrated trailing edges." Journal of Fluid Mechanics 793 (2016): 556-588. 
[47] Fassmann, Benjamin, et al. "Efficient prediction of broadband trailing edge noise and application to porous edge treatment." arXiv preprint arXiv:1810.02642 (2018).

[48] Lee, Seongkyu. "Empirical wall-pressure spectral modeling for zero and adverse pressure gradient flows." AIAA Journal 56.5 (2018): 1818-1829.

[49] Howe, Michael S., and Michael S. Howe. Acoustics of fluid-structure interactions. Cambridge university press, 1998.

[50] Liu, Chen, and Seongkyu Lee. "Parametric Airfoil Design for Trailing-Edge Noise Reduction." AIAA AVIATION 2020 FORUM. 2020.

[51] Cao, Huijing, et al. "Flow topology and noise modeling of trailing edge serrations." Applied Acoustics 168 (2020): 107423.

[52] Sandberg, R. D., and L. E. Jones. "Direct numerical simulations of low Reynolds number flow over airfoils with trailing-edge serrations." Journal of Sound and Vibration 330.16 (2011): 3818-3831.

[53] Jawahar, Hasan Kamliya, Yujing Lin, and Mark Savill. "Large eddy simulation of airfoil self-noise using OpenFOAM." Aircraft Engineering and Aerospace Technology (2018).

[54] Kamliya Jawahar, Hasan, Qing Ai, and Mahdi Azarpeyvand. "Experimental and numerical investigation of aerodynamic performance of airfoils fitted with morphing trailing-edges." 23rd AIAA/CEAS aeroacoustics conference. 2017.

[55] Wolf, William R., and Sanjiva K. Lele. "Trailing-edge noise predictions using compressible large-eddy simulation and acoustic analogy." AIAA journal 50.11 (2012): 2423-2434.

[56] Manoha, Eric, Bruno Troff, and Pierre Sagaut. "Trailing-edge noise prediction using large-eddy simulation and acoustic analogy." AIAA journal 38.4 (2000): 575-583.

[57] Agrawal, Bharat R., and Anupam Sharma. "Aerodynamic noise prediction for a rod-airfoil configuration using large eddy simulations." 20th AIAA/CEAS Aeroacoustics Conference. 2014. 
[58] Tang, Hui, Yulong Lei, and Yao Fu. "Noise Reduction Mechanisms of an Airfoil with Trailing Edge Serrations at Low Mach Number." Applied Sciences 9.18 (2019): 3784.

[59] Zilstra, Alison, and David Johnson. "LES and FW-H Prediction of Aeroacoustic Noise for a SD 7037 Airfoil for Wind Turbine Applications." 25th AIAA/CEAS Aeroacoustics Conference. 2019.

[60] Quéméré, P., and P. Sagaut. "Zonal multi-domain RANS/LES simulations of turbulent flows." International journal for numerical methods in fluids 40.7 (2002): 903-925.

[61] Terracol, Marc. "A zonal RANS/LES approach for noise sources prediction." Flow, turbulence and combustion 77.1-4 (2006): 161-184.

[62] Fröhlich, Jochen, and Dominic Von Terzi. "Hybrid LES/RANS methods for the simulation of turbulent flows." Progress in Aerospace Sciences 44.5 (2008): 349-377.

[63] Mathey, Fabrice. "Aerodynamic noise simulation of the flow past an airfoil trailing-edge using a hybrid zonal RANS-LES." Computers \& Fluids 37.7 (2008): 836-843.

[64] Kim, Taehyung, Jeon, Minu, Lee, Soogab, and Shin Hyungki. "Numerical Simulation of Flatback Airfoil Aerodynamic Noise.” Renewable Energy, vol. 65, 2014, pp. 192-201.

[65] Lane, Graeme, Paul Croaker, and Yan Ding. "Embedded Large Eddy Simulation Method for Predicting Flow-Induced Noise." Proceedings of ACOUSTICS. Vol. 10. No. 13. 2019.

[66] Zuo, Z., Q. Huang, and S. Liu. "An analysis on the flow field structures and the aerodynamic noise of airfoils with serrated trailing edges based on embedded large eddy flow simulations." Journal of Applied Fluid Mechanics 12.2 (2019): 327-339.

[67] Romani, Gianluca, Damiano Casalino, and Wouter van der Velden. "Numerical Analysis of Airfoil Trailing-Edge Noise for Straight and Serrated Edges at Incidence." AIAA Journal (2021): 1-20. 
[68] Farassat, F., and George P. Succi. "The prediction of helicopter rotor discrete frequency noise." In: American Helicopter Society (1982): 497-507.

[69] Hinze, J. Turbulence (McGraw-Hill Series in Mechanical Engineering). 2nd ed., McGraw-Hill College, 1975.

[70] Jones, W. P., and Brian Edward Launder. "The prediction of laminarization with a two-equation model of turbulence." International journal of heat and mass transfer 15.2 (1972): 301-314.

[71] Launder, Brian Edward, and Bahrat I. Sharma. "Application of the energydissipation model of turbulence to the calculation of flow near a spinning disc." Letters in heat and mass transfer 1.2 (1974): 131-137.

[72] Wilcox, David C. Turbulence modeling for CFD. Vol. 2. La Canada, CA: DCW industries, 1998.

[73] Menter, Florian R. "Two-equation eddy-viscosity turbulence models for engineering applications." AIAA journal 32.8 (1994): 1598-1605.

[74] Smagorinsky, Joseph. "General circulation experiments with the primitive equations: I. The basic experiment." Monthly weather review 91.3 (1963): 99164.

[75] Zhiyin, Yang. "Large-eddy simulation: Past, present and the future." Chinese journal of Aeronautics 28.1 (2015): 11-24.

[76] Fluent Theory Guide - 2020 R2.

[77] Leonard, Athony. "Energy cascade in large-eddy simulations of turbulent fluid flows." Advances in geophysics. Vol. 18. Elsevier, 1975. 237-248.

[78] Nicoud, Franck, and Frédéric Ducros. "Subgrid-scale stress modelling based on the square of the velocity gradient tensor." Flow, turbulence and Combustion 62.3 (1999): 183-200.

[79] Mathey, Fabrice, et al. "Assessment of the vortex method for large eddy simulation inlet conditions." Progress in Computational Fluid Dynamics, An International Journal 6.1-3 (2006): 58-67.

[80] Van Blitterswyk, Jared, and Joana Rocha. "Prediction and measurement of flow-induced wall-pressure fluctuations at low Mach numbers." Canadian Acoustics 42.4 (2014): 3-14. 
[81] Al Tlua, Basim, and Joana Rocha. "Development and Testing of an Aeroacoustic Wind Tunnel Test Section." Canadian Acoustics 47.3 (2019): 6465.

[82] Al Tlua, Basim, and Rocha Joana. "Optimization and Testing of Flat-Plate Trailing-Edge Serration Geometry for Reducing Airfoil Self-Noise." Canadian Acoustics 48.4 (2020): 7-18.

[83] Berger, Marsha, and Michael Aftosmis. "Progress towards a Cartesian cut-cell method for viscous compressible flow." 50th AIAA Aerospace Sciences Meeting Including the New Horizons Forum and Aerospace Exposition. 2012.

[84] Ghmati, Ramadan Elhadi, Badih Ali Jawad, and Evangelos Koutsavdis. "An Investigation of CutCell Meshing Strategies for Accurate Aerodynamic Performance Prediction." SAE International Journal of Passenger CarsMechanical Systems 5.2012-01-0499 (2012): 369-380.

[85] Menter, Florian R. "Best practice: scale-resolving simulations in ANSYS CFD." ANSYS Germany GmbH 1 (2012).

[86] ANSYS Fluent 2019 User Guide, ANSYS, Inc 2019.

[87] Goetten, Falk, et al. "A review of guidelines and best practices for subsonic aerodynamic simulations using RANS CFD." APISAT 2019: Asia Pacific International Symposium on Aerospace Technology. Engineers Australia, 2019.

[88] Press, William H., Flannery, B. P., Teukolsky, S. A., and Vetterling, W. T., "Numerical recipes." (1989).

[89] Clauser, Francis H. "Turbulent boundary layers in adverse pressure gradients." Journal of the Aeronautical Sciences 21.2 (1954): 91-108.

[90] Falkneb, V. M., and Sylvia W. Skan. "LXXXV. Solutions of the boundarylayer equations." The London, Edinburgh, and Dublin Philosophical Magazine and Journal of Science 12.80 (1931): 865-896.

[91] Ludwieg, H., and Tillmznn, W. Investigations of the wall shearing stress in turbulent boundary layers. NACA T.M., 1950, no. 1285.

[92] Lee, H., and S-H. Kang. "Flow characteristics of transitional boundary layers on an airfoil in wakes." J. Fluids Eng. 122.3 (2000): 522-532. 
[93] Suslick, Kenneth S. "Encyclopedia of physical science and technology." Sonoluminescence and Sonochemistry Massachusetts: Elsevier Science Ltd (2001): 1-20.

[94] Marsden, Olivier, Christophe Bogey, and Christophe Bailly. "Direct Noise Computation around a 3-D NACA 0012 airfoil." 12th AIAA/CEAS Aeroacoustics Conference (27th AIAA Aeroacoustics Conference). 2006.

[95] Amiet, Roy K. "Noise due to turbulent flow past a trailing edge." Journal of sound and vibration 47.3 (1976): 387-393.

[96] Williams, JE Ffowcs, and L. H. Hall. "Aerodynamic sound generation by turbulent flow in the vicinity of a scattering half plane." Journal of fluid mechanics 40.4 (1970): 657-670.

[97] JCR, Hunt, A. Wray, and P. Moin. "Eddies, stream, and convergence zones in turbulent flows." Studying Turbulence Using Numerical Simulation Databases-I1 193 (1988).

[98] Chong, Min S., Anthony E. Perry, and Brian J. Cantwell. "A general classification of three-dimensional flow fields." Physics of Fluids A: Fluid Dynamics 2.5 (1990): 765-777.

[99] Banko, A. J., and J. K. Eaton. "A frame-invariant definition of the Q-criterion." Center for Turbulence Research Annual Research Briefs (2019): 181-194.

[100] Green, R. B., and J. H. Gerrard. "Vorticity measurements in the near wake of a circular cylinder at low Reynolds numbers." Journal of Fluid Mechanics 246 (1993): 675-691.

[101] Gerrard, J. H. "The mechanics of the formation region of vortices behind bluff bodies." Journal of fluid mechanics 25.2 (1966): 401-413.

[102] Strouhal, Vincenz. Über eine besondere Art der Tonerregung. Stahel, 1878.

[103] Salama, Y., \& Rocha, J. (2021, August). ELES and Tonal Noise Prediction of Slanted-Root Trailing-Edge Serrations. Conference: 50th International Congress and Exposition on Noise Control Engineering (Inter-Noise 2021, Washington, DC, USA.

[104] R. Dieck, W. Steele, and G. Osolsobe, Test uncertainty. ASME ptc 19.12005, American Society of Mechanical Engineers, New York, NY, 2005. 


\section{Appendix A}

\section{Measurement Uncertainty Analysis}

The uncertainty of any measurement comes from two sources of errors:

1. Systematic, bias, error related to the hardware used to acquire data, such as the data acquisition system, microphone junction, extension wires, and connectors. This type of error is usually constant throughout the experiment.

2. Random error, which is inversely proportional to the number of experiment repetitions at the same condition.

This section explains the method used to calculate random uncertainty and presents types of bias errors considered. The method used is described in detail by the American Society of Mechanical Engineers (ASME) [104], such that

$$
T U_{A S M E}= \pm t_{95}\left[\left(\frac{B_{T}}{2}\right)^{2}+\left(R_{T}\right)^{2}\right]^{0.95}
$$

where $T U_{A S M E}$ is the total uncertainty of the measurement, $B_{T}$ is the total bias uncertainty, $R_{T}$ is the total random uncertainty, and $t_{95}$ is the value at $95 \%$ confidence. For a number of repeats equal to 5 and having a degree of freedom of $N-1=4, t_{95}=2.776$. The total bias uncertainty $\left(B_{T}\right)$ is calculated using the root sum squared, as seen in equation A2. The obtained values are listed in Table A1.

$$
B_{T}=\sqrt{B_{1}^{2}+B_{2}^{2}+B_{3}^{2}+B_{4}^{2}}
$$

Lastly, the total random uncertainty $\left(R_{T}\right)$ is found using standard deviation, such that 


$$
R_{T}=\sqrt{\frac{\sum_{i=1}^{N}\left(P_{i}-\bar{P}\right)^{2}}{N-1}}
$$

where $N$ is the number of repeats, $P_{i}$ is microphone reading noise level in $\mathrm{dB}$, and $P$ is the mean of microphone readings noise level in $\mathrm{dB}$. An estimate for the uncertainty in the noise level is made based on repeated measurements. Several measurements of the noise were made over a range of test section velocities. The uncertainties presented are averaged over the entire frequency range $(0.1 \mathrm{kHz}-$ $10 \mathrm{kHz}$ ). Table A.2 presents the calculated uncertainties for noise levels as a function of flow speed.

Table A1: Sources of systematic uncertainty and total bias uncertainty.

\begin{tabular}{ccc}
\hline Hardware Component & Source Error Type & Bias Uncertainty $( \pm \%)$ \\
\hline Data Acquisition System & $\left(B_{1}\right)$ & 0.10 \\
\hline \multirow{2}{*}{ Microphone } & Mic. accuracy $\left(B_{2}\right)$ & 0.83 \\
\cline { 2 - 3 } & Mic. extension wire $\left(B_{3}\right)$ & 0.33 \\
\hline Mic. connector & Mic. connector $\left(B_{4}\right)$ & 0.07 \\
\hline \multicolumn{2}{c}{ Total Bias Uncertainty $\left(\boldsymbol{B}_{\boldsymbol{T}}\right)$} & 0.81 \\
\hline
\end{tabular}

Table A2: Uncertainty in noise levels.

\begin{tabular}{cc}
\hline Flow velocity $(\boldsymbol{m} / \boldsymbol{s})$ & Uncertainty $( \pm \boldsymbol{d B})$ \\
\hline 7 & 1.12 \\
\hline 9 & 1.18 \\
\hline 12.7 & 1.25 \\
\hline 14 & 1.30 \\
\hline 16 & 1.36 \\
\hline 18 & 1.40 \\
\hline 22 & 1.47 \\
\hline 24 & 1.51 \\
\hline 26.7 & 1.58
\end{tabular}

